\title{
Pillar[5]arene-Decorated Single-Walled Carbon
}

\author{
Nanotubes
}




\title{
PILLAR[5]ARENE-DECORATED SINGLE-WALLED CARBON NANOTUBES
}

BY

CHRISTINA SHAMSHOOM, B.Sc.

\begin{abstract}
A THESIS
SUBMITTED TO THE DEPARTMENT OF CHEMISTRY AND CHEMICAL BIOLOGY AND THE SCHOOL OF GRADUATE STUDIES

OF MCMASTER UNIVERSITY
\end{abstract}

IN PARTIAL FULFILMENT OF THE REQUIREMENTS

FOR THE DEGREE OF

M.SC. IN CHEMISTRY

(C) Copyright by Christina Shamshoom, September 2018

All Rights Reserved 
M.Sc. Thesis - C. Shamshoom - McMaster University- Chemistry and Chemical Biology

MSc of Chemistry (2018)

(Chemistry and Chemical Biology)
McMaster University

Hamilton, Ontario, Canada

TITLE: Pillar[5]arene-Decorated Single-Walled Carbon Nanotubes

AUTHOR: Christina Shamshoom

B.Sc., (Chemistry)

University of Guelph, Guelph, Canada

SUPERVISOR: Professor Alex Adronov

NUMBER OF PAGES: ix, 89 
M.Sc. Thesis - C. Shamshoom - McMaster University- Chemistry and Chemical Biology

\section{Abstract}

Control of single-walled carbon nanotube dispersion properties is of substantial interest to the scientific community. In this work, we sought to investigate the effect of a macrocycle, the pillar[5]arene, on the dispersion properties of a polymer-nanotube complex. Pillar[5]arenes are a class of electron-rich macrocyclic hosts capable of forming inclusion complexes with electron-poor guests, such as alkyl nitriles. A hydroxylfunctionalized pillar[5] arene derivative was coupled to the alkyl bromide side-chains of a polyfluorene, which was then used to coat the surface of single-walled carbon nanotubes. Differentiation of semiconducting and metallic SWNT species was analyzed by a combination of UV-Vis-NIR, Raman, and fluorescence spectroscopy. Raman spectroscopy confirmed that the concentrated nanotube dispersion produced by the macrocyclecontaining polymer was due to well-exfoliated nanotubes, rather than bundle formation.

The polymer-nanotube dispersion was investigated using ${ }^{1} \mathrm{H}-\mathrm{NMR}$ spectroscopy, and it was found that host-guest chemistry between pillar[5]arene and 1,6-dicyanohexane occurred in the presence of the polymer-nanotube complex. Utilizing the host-guest capability of pillar[5]arene, the polymer-nanotube complex was incorporated into a supramolecular organogel. 
M.Sc. Thesis - C. Shamshoom - McMaster University- Chemistry and Chemical Biology

\section{Acknowledgements}

Throughout my M.Sc. degree, I have had the opportunity to work with numerous individuals who have helped me to achieve my academic goals. First, I would like to thank my academic supervisor Dr. Alex Adronov for taking me on as a student and guiding me throughout my degree. Under your supervision, I gained immense scientific knowledge and valuable research experience that I will take with me as I move forward in my career. Also, I would like to thank my supervisory committee, Dr. Ryan Wylie and Dr. Harald Stöver for their suggestions and helpful discussions.

The following thesis would not have been possible without the help of numerous individuals in the Adronov group: Dr. Nicole Rice, Stuart McNelles, Vladimir Kardelis, Darryl Fong, Kelvin Li, William J. Bodnaryk, and Dr. Eric Meichsner. I am truly indebted to all of you for the training, support, and guidance you offered over the past two years. I will always cherish the laughter, fun times, and comradery we shared. It has been a pleasure getting to know all of you and working alongside you in lab. Thank you for being there for me when my experiments did not work and offering advice despite your busy schedules.

Most importantly, I would like to express my deep gratitude towards my parents and siblings for their endless support and unconditional love. You have been truly instrumental in all my achievements, and without you, my university degrees would not have been possible. 
M.Sc. Thesis - C. Shamshoom - McMaster University- Chemistry and Chemical Biology

\section{Table of Contents}

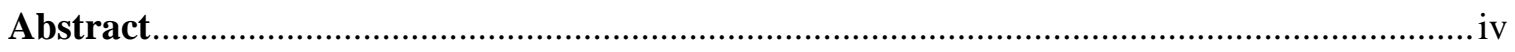

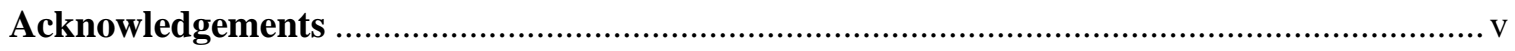

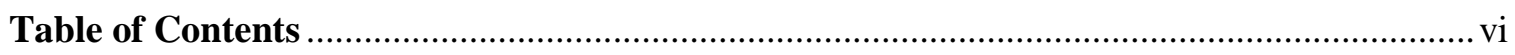

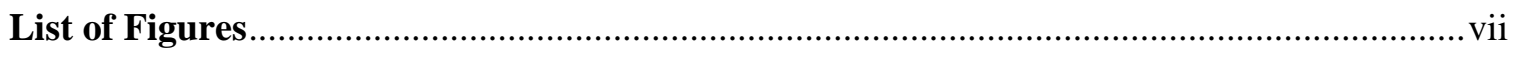

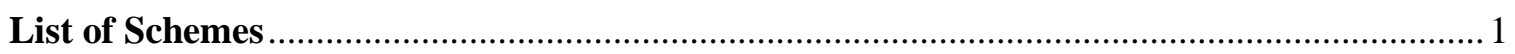

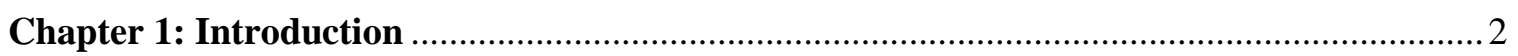

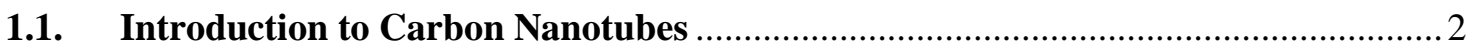

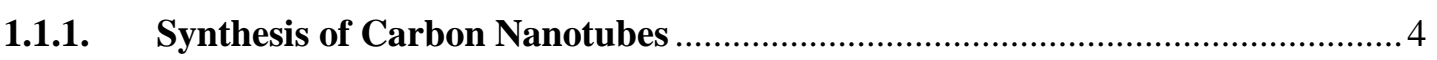

1.2. Solubilization of Carbon Nanotube Bundles ........................................................ 6

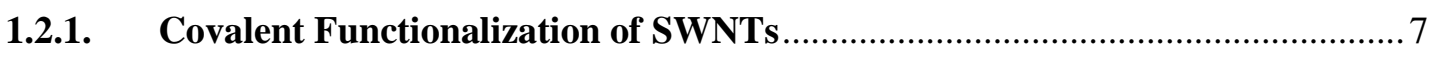

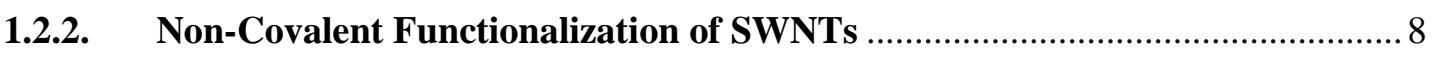

1.2.3. Conjugated Polymers for Non-covalent Functionalization of SWNTs............ 12

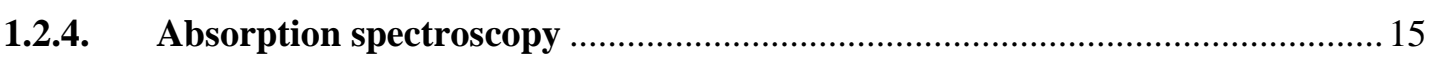

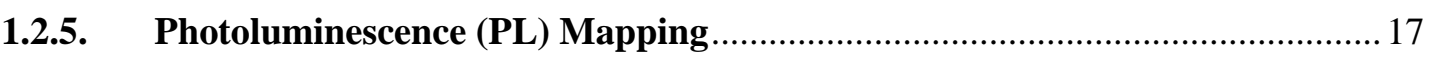

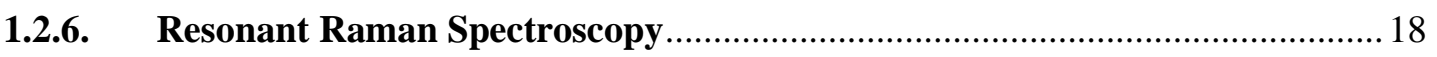

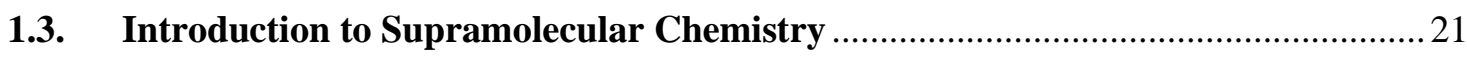

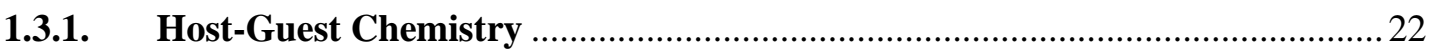

1.3.2. Introduction to Pillar[5]

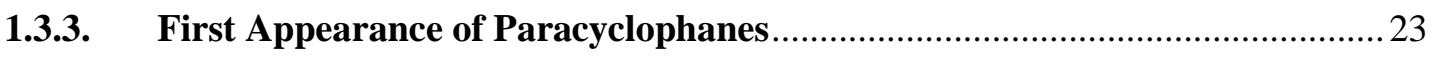

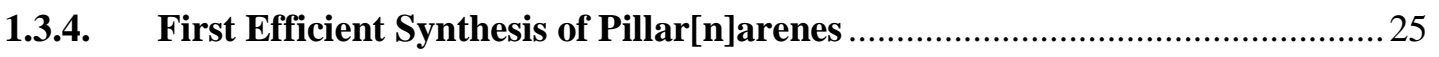

1.3.5. Synthesis of Pillar[5]arenes under Thermodynamic Control .........................26

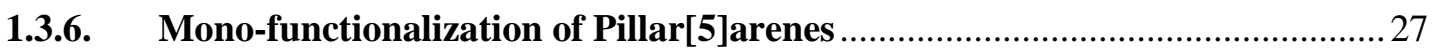

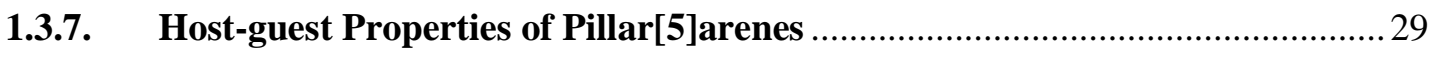

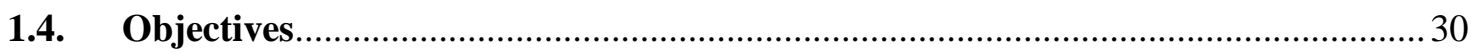

Chapter 2: Preparation and Characterization of Polymer-SWNT Complexes...................... 33

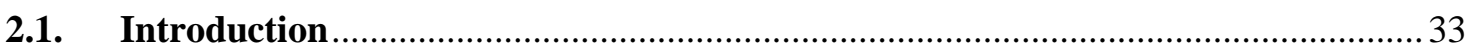

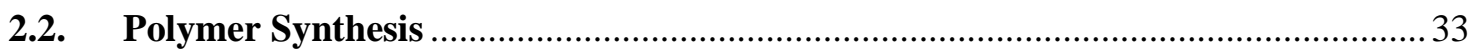

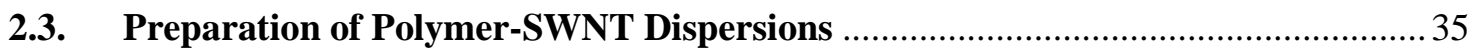

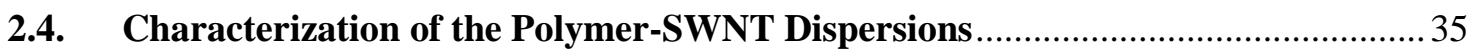

2.5. Host-Guest Properties of Polymer-SWNT Dispersion.......................................... 40

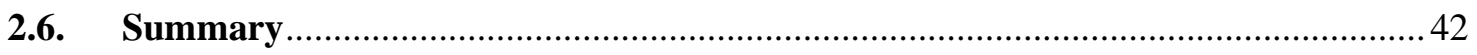

Chapter 3: Preparation and Characterization of Supramolecular Organogels..................... 44 
M.Sc. Thesis - C. Shamshoom - McMaster University- Chemistry and Chemical Biology

3.1. Preparation of Supramolecular Organogels............................................................ 45

3.2. Preparation of Organogels Utilising Solvent Exchange Method ............................. 48

3.3. Mechanical Testing of Organogels Utilising the CellScale Microsquisher .............52

3.4. Mechanical Testing of Organogels using a Home-built Apparatus........................57

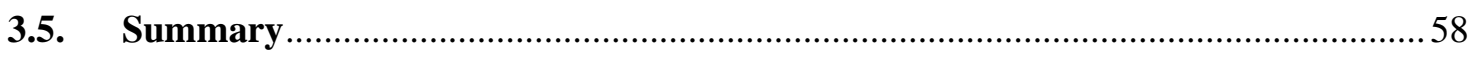

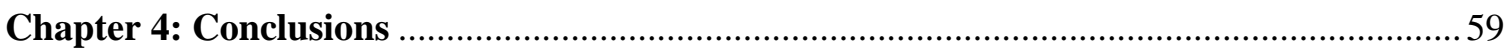

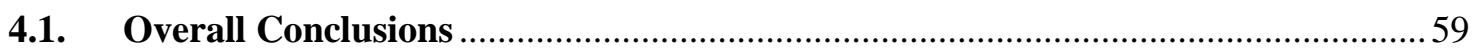

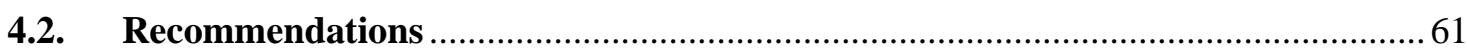

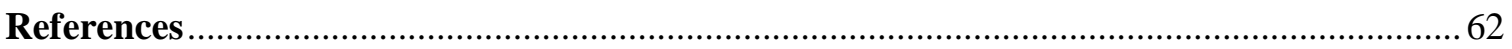

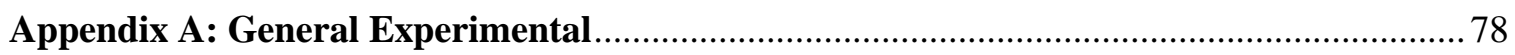

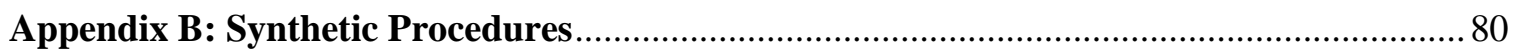

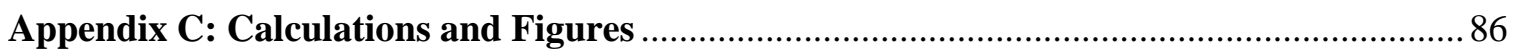

\section{List of Figures}

Figure 1. Various examples of carbon allotropes. Adapted from [Rice, N. A. Separation of Single-Walled Carbon Nanotubes By Electronic Type Using Conjugated Polymers,

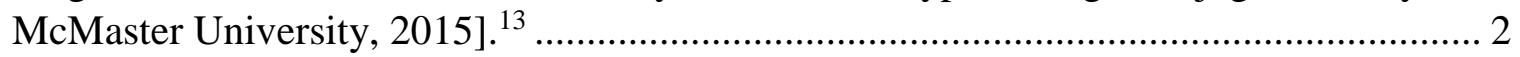

Figure 2. The "roll-up" of a graphene sheet which generates zigzag (blue), armchair (red), and chiral (gray) SWNTs. Reproduced with permission from [Balasubramanian, K.; Burghard, M. Small 2005, 1 (2), 180-192]. ${ }^{15}$ Copyright John Wiley \& Sons, 2004......... 4

Figure 3. Idealized representation of SWNTs in a bundle. Reproduced with permission from [Hirsch, A. Angew. Chemie - Int. Ed. 2002, 41 (11), 1853-1859]. ${ }^{30}$ Copyright John Wiley \& Sons, 2002. 7

Figure 4. Structures of reported surfactants used in the individualization of SWNTs: sodium dodecyl sulfate (SDS), sodium dodecylbenezenesulfonate (SDBS), sodium cholate (SCh), and deoxycholate (DOC). 9

Figure 5. A photograph (left) of a centrifuge tube containing several bands of SWNTs sorted by the density gradient ultracentrifugation (DGU) technique, and their corresponding UV-Vis-NIR absorption spectra (right). Reproduced with permission from 
M.Sc. Thesis - C. Shamshoom - McMaster University- Chemistry and Chemical Biology

[Ghosh, S.; Bachilo, S. M.; Weisman, R. B. Nat. Nanotechnol. 2010, 5 (6), 443-450]. ${ }^{59}$

Copyright Nature Group, 2009. 11

Figure 6. UV-Vis-NIR absorption spectra of 12 sc-SWNT species isolated via DNA and IEX and raw HiPCO SWNTs (top black spectrum). Reproduced with permission from [Tu, X.; Manohar, S.; Jagota, A.; Zheng, M. Nature 2009, 460 (7252), 250-253].${ }^{61}$ Copyright Nature Publishing Group, 2009. 12

Figure 7. Schematic representation of the various optical processes of SWNTs, such as (a) absorbance, (b) Rayleigh scattering, (c) photoluminescence (PL), and (d) Stokes Raman processes. Reproduced with permission from [Rice, N. A. Separation of Single-Walled Carbon Nanotubes By Electronic Type Using Conjugated Polymers, McMaster University, 2015]. ${ }^{13}$ Copyright 2015. 15

Figure 8. Semiempirical Kataura plot of $S_{11}$ (black), $S_{22}$ (blue) and $M_{11}$ (red). The electronic transitions data for sc-SWNT ${ }^{74}$ and $\mathrm{m}-\mathrm{SWNT}^{80,81}$ were obtained from literature sources. The orange box depicts the expected diameter range for HiPCO SWNTs (diameter range from $\sim 0.7$ to $1.3 \mathrm{~nm}$ ). The horizontal lines denote the three laser excitation wavelengths $(514,633$, and $785 \mathrm{~nm})$ used for Raman studies in this thesis. Reproduced with permission from [Rice, N. A. Separation of Single-Walled Carbon Nanotubes By Electronic Type Using Conjugated Polymers, McMaster University, 2015]. ${ }^{13}$ Copyright 2015. 19

Figure 9. A typical Raman spectrum for a SWNT sample, showing the RBM (red), D-band (blue), the G-band (orange), and G'-band (green) regions. 20

Figure 10. Schematic representation for the proposed pathway of pillar[5]arene under thermodynamic control. 27

Figure 11. ${ }^{1} \mathrm{H}-\mathrm{NMR}$ spectra overlay of PF-Br (blue) and PF-Pillar (orange) in $\mathrm{CDCl}_{3 .} .34$

Figure 12. Protocol for preparation of polymer-wrapped-SWNT dispersions in organic solvent. 35

Figure 13. UV-Vis-NIR absorption spectra of polymer-SWNT dispersions (2:1 polymer:SWNT mass ratio) in THF for PF-Pillar-SWNT (orange) and PF-Br-SWNT (blue). The absorption spectrum for PF-Pillar-SWNT was diluted five-fold in THF. .... 36 
M.Sc. Thesis - C. Shamshoom - McMaster University- Chemistry and Chemical Biology

Figure 14. RBM regions at (a) 514, (b) 633, and (c) $785 \mathrm{~nm}$ excitation wavelengths. Raman spectra The gray boxes denote the signals arising from sc-SWNTs, while the pink boxes indicate the locations of signals from m-SWNTs. The inset in (a) shows the G-band region, located at $\sim 1590 \mathrm{~cm}^{-1}$, upon excitation at $514 \mathrm{~nm}$.

Figure 15. Photoluminescence maps of (a) PF-Br-SWNT and (b) PF-Pillar-SWNT, concentration-matched by UV-Vis-NIR and plotted on the same scale. 40

Figure 16. ${ }^{1} \mathrm{H}-\mathrm{NMR}$ spectra $\left(\mathrm{THF}_{8} \mathrm{~d}_{8}, 298 \mathrm{~K}\right.$ ) of the PF-Pillar-SWNT dispersion recorded after successive additions of 1,6-dicyanohexane $(0-65$ eq) 42

Figure 17. Cartoon representation of host-guest driven gelation of PF-Pillar (purple) and PF-CN (green). Adapted from [Kardelis, V.; Li, K.; Nierengarten, I.; Holler, M.; Nierengarten, J. F.; Adronov, A. Macromolecules 2017, 50 (23), 9144-9150]. ${ }^{166}$......... 45

Figure 18. Cartoon representation of host-guest driven gelation of PF-Pillar-SWNT (red) and $\mathrm{PEG}_{600}-(\mathrm{CN})_{2}$ (green).

Figure 19. RBM regions of the Raman spectra of PF-Pillar-SWNT samples in THF and upon solvent exchange into 1,2-dichlorobenzene (DCB), compared with raw HiPCO SWNTs. The gray and pink boxes denote the regions of integrated bundling peaks and other SWNT chirlaities, respectively. The spectra were collected at $785 \mathrm{~nm}$ excitation wavelength, normalized to the G-band at $\sim 1590 \mathrm{~cm}^{-1}$ and offset for clarity. 50

Figure 20. Photographs of supramolecular organogels made from the host-guest interaction between PF-Pillar and PEG600- $(\mathrm{CN})_{2}$ (native) and in the presence of SWNTs (hybrid). 52

Figure 21. Photograph of the CellScale Microsquisher. Adapted from [CellScale. MicroSquisher Micro-scale Tension-Compression Test System]. ${ }^{178}$ 53

Figure 22. Test sequence showing the load, hold, recover, and rest phases using the CellScale Microsquisher. Adapted from [CellScale. MicroSquisher Micro-scale TensionCompression Test System]. ${ }^{178}$ 55 
M.Sc. Thesis - C. Shamshoom - McMaster University- Chemistry and Chemical Biology

\section{List of Schemes}

Scheme 1. Structures of various paracyclophanes.

Scheme 2. The macrocycles obtained during the selective reduction of 4

Scheme 3. Multi-step synthesis of paracyclophanes 1, 2, and 3.

Scheme 4. First efficient synthesis of pillar[5]arene reported by Ogoshi in 2008. 26

Scheme 5. Two approaches for the mono-functionalization of pillar[5]arenes.

Scheme 6. Post-polymerization functionalization of PF-Br with 6 to afford PF-Pillar. 34

Scheme 7. Various attempts to synthesize polyfluorene containing alkyl nitrile side chains (PF-CN) via cyanide substitution of the alkyl bromide on polyfluorene bromide (PF-Br). 
M.Sc. Thesis - C. Shamshoom - McMaster University- Chemistry and Chemical Biology

\section{Chapter 1: Introduction}

\subsection{Introduction to Carbon Nanotubes}

Immediately following the synthesis of fullerenes on a macroscopic scale, ${ }^{1}$ the Japanese physicist Sumio Iijima accidentally discovered carbon nanotubes on the surface of graphite electrodes used in an electric arc discharge. ${ }^{2}$ Carbon nanotubes are hollow, tubular allotropes of carbon that are approximately $1 \mathrm{~nm}$ in diameter and roughly $1 \mu \mathrm{m}$ in length. ${ }^{3}$ As shown in Figure 1, carbon nanotubes may be composed of one layer of carbon atoms to form a single-walled carbon nanotube (SWNT), or multiple SWNTs of different diameters placed inside one another to form a multi-walled carbon nanotube (MWNT). Since their discovery in $1993,{ }^{2}$ single-walled carbon nanotubes (SWNTs) have attracted significant interest within the scientific community because of their extraordinary structural, ${ }^{4-6}$ mechanical, ${ }^{7-9}$ and optoelectronic properties. ${ }^{10-12}$
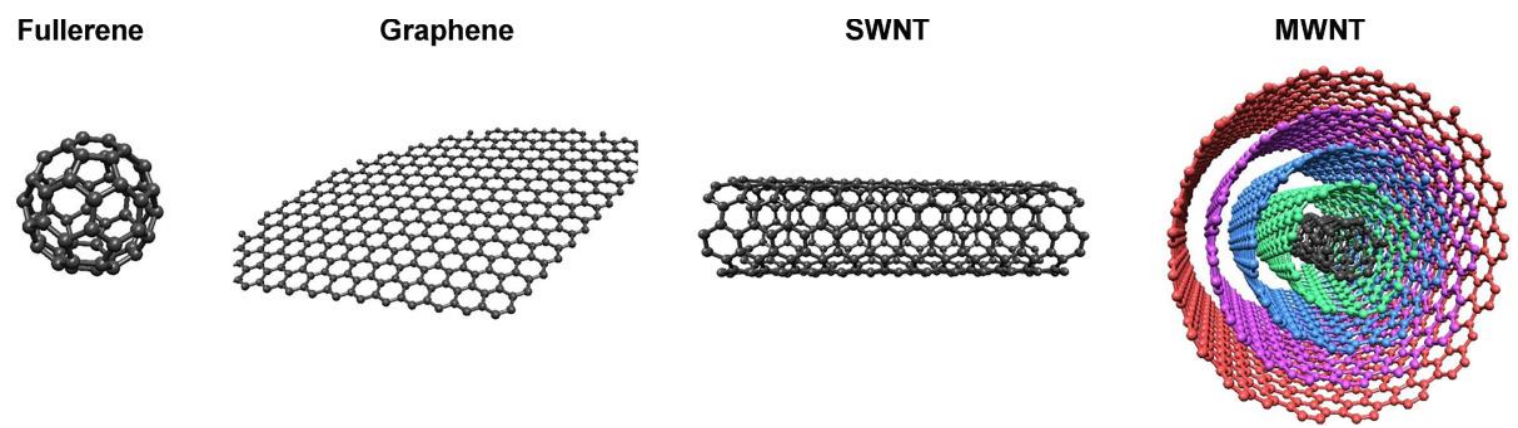

Figure 1. Various examples of carbon allotropes. Adapted from [Rice, N. A. Separation of Single-Walled Carbon Nanotubes By Electronic Type Using Conjugated Polymers, McMaster University, 2015]. ${ }^{13}$

Theoretically, SWNTs can be imagined as a graphene sheet that is rolled up to form a cylinder, as shown in Figure 2. The different permutations of the two-dimensional "rolled up" graphene lattice generates an assortment of quasi-one-dimensional nanotube species such as zigzag, armchair, and helical (commonly known as chiral) SWNTs. ${ }^{14}$ The 
M.Sc. Thesis - C. Shamshoom - McMaster University- Chemistry and Chemical Biology

numerous ways in which a graphene sheet is rolled into a carbon nanotube may be defined mathematically by the chiral vector equation:

$$
C_{h}=n a_{1}+m a_{2}=d_{t} \pi
$$

Where $n$ and $m$ are integers which correspond to the lattice translational indices, and $\mathrm{a}_{1}$ and $\mathrm{a}_{2}$ are unit vectors for the graphene lattice (Figure 2). The chiral vector is also related to the SWNT diameter $\left(\mathrm{d}_{\mathrm{t}}\right)$ because it encompasses the circumference of the rolledup nanotube. ${ }^{14}$ Carbon nanotubes are also described by the chiral angle, $\theta$, which is defined as the angle between the vectors $C_{h}$ and $\mathrm{a}_{1}$ as shown by the following equation:

$$
\theta=\tan ^{-1}\left[\frac{\sqrt{3 m}}{(2 n+m)}\right]
$$

The values of chiral angle are restricted to $0-30^{\circ}$ due to the hexagonal symmetry of the graphene lattice. The values on either extreme of the range (i.e. $0^{\circ}$ and $30^{\circ}$ ) are present in achiral SWNTs. Thus, a given nanotube is zigzag if $\theta=0^{\circ}(m=0)$, while if $\theta=$ $30^{\circ}(n=m)$ it is known as an armchair SWNT. As shown in Figure 2, having a chiral vector perpendicular to the $\mathrm{C}=\mathrm{C}$ bond directions (shown in blue) will generate a zigzag SWNT, denoted as $(n, 0) .{ }^{14}$ Conversely, if the chiral vector is parallel to the direction of the $\mathrm{C}=\mathrm{C}$ bond (shown in red), it will result in an armchair SWNT. ${ }^{14}$ The chiral angle values in between 0 and $30^{\circ}$ are present in chiral SWNTs that lack mirror symmetry. 
M.Sc. Thesis - C. Shamshoom - McMaster University- Chemistry and Chemical Biology

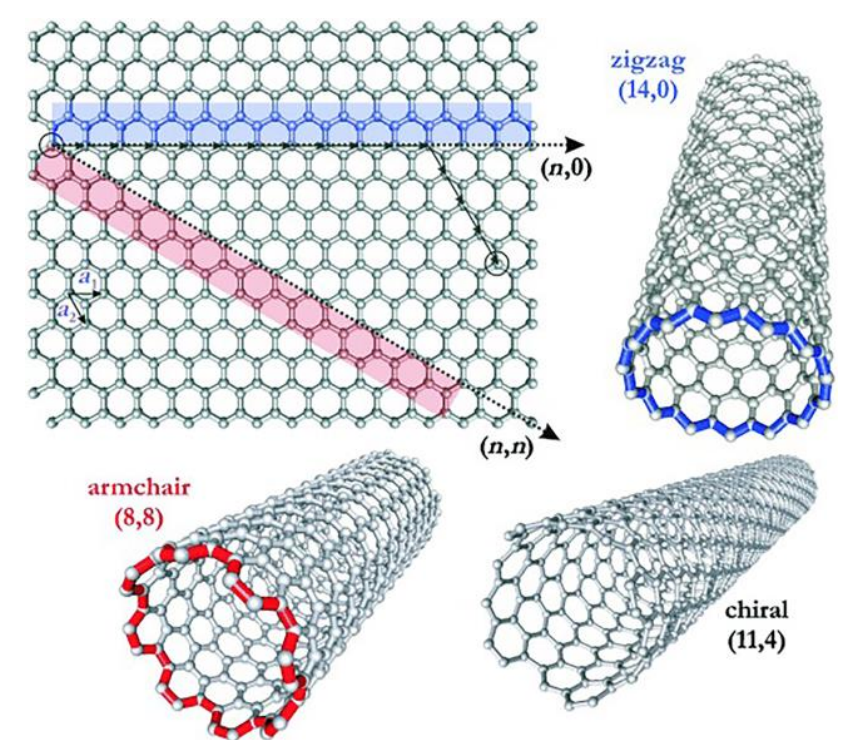

Figure 2. The "roll-up" of a graphene sheet which generates zigzag (blue), armchair (red), and chiral (gray) SWNTs. Reproduced with permission from [Balasubramanian, K.; Burghard, M. Small 2005, 1 (2), 180-192]. ${ }^{15}$ Copyright John Wiley \& Sons, 2004.

\subsubsection{Synthesis of Carbon Nanotubes}

Commercial carbon nanotubes are synthesized by various techniques, including arc discharge, laser ablation, chemical vapor deposition (CVD), high-pressure carbon monoxide disproportionation (HiPCO), and a CVD variant that uses a cobalt-molybdenum catalyst called CoMoCAT. ${ }^{16}$ In 1991, Iijima reported the first synthesis of carbon nanotubes (MWNTs) via an arc discharge evaporation method, similar to fullerene synthesis. ${ }^{2}$ The resultant needle-like tubes were approximately 4 to $30 \mathrm{~nm}$ in diameter and about $1 \mu \mathrm{m}$ in length. ${ }^{2}$ The carbon nanotubes were grown on the negative end of a carbon electrode inside a pressurized chamber composed of a mixture of methane and argon gas, at pressures of 10 Torr and 40 Torr, respectively. Inside the pressurized chamber, a current was applied across a metal catalyst-impregnated cathode and electrode, resulting in carbon 
M.Sc. Thesis - C. Shamshoom - McMaster University- Chemistry and Chemical Biology

nanotubes that grew on the catalyst particles. ${ }^{17}$ Hence, arc-discharge evaporation is the oldest reported method used for fabrication of carbon nanotubes in macroscopic amounts. ${ }^{2}$ Another method for the bulk synthesis of SWNTs, known as laser ablation, was first introduced by Smalley and co-workers in $1995 .{ }^{18}$ It involves direct laser vaporization of a transition metal-graphite target in a high temperature reactor that contains an inert gas. ${ }^{18}$ The gas phase carbon rises and as it reaches the cold surface of the reactor walls, the carbon condenses to grow carbon nanotubes. While the fundamental nanotube growth mechanism is akin to the arc-discharge method, direct vaporization has some advantages such as improved control over growth conditions and it efficiently generates nanotubes in higher yield (up to 70\%). ${ }^{18}$ In addition, the laser ablation method affords more control in the diameter of the carbon nanotubes synthesized. ${ }^{19}$

CVD is another synthetic method of carbon nanotubes which was developed by Xie and co-workers in $1996 .^{20}$ This method involves the catalytic decomposition of a hydrocarbon gas (such as methane, ethene and ethyne) on a solid catalyst in a high temperature $\left(700\right.$ to $\left.900^{\circ} \mathrm{C}\right)$ chamber.

The HiPCO method was first developed by researchers at Rice University in 1999, where carbon nanotubes are synthesized in a high pressure and high temperature (700 to $900^{\circ} \mathrm{C}$ ) chamber. It is a scalable, continuous process which generates sizeable amounts of SWNTs formed free of a catalyst support. This method is similar to CVD, with the exception that the catalyst is introduced in the gas phase as opposed to the solid state. The HiPCO method utilizes carbon monoxide gas as a carbon source and iron pentacarbonyl 
M.Sc. Thesis - C. Shamshoom - McMaster University- Chemistry and Chemical Biology

$\left(\mathrm{Fe}(\mathrm{CO})_{5}\right)$ catalyst, where the iron forms metal clusters that act as catalytic sites on which small diameter carbon nanotubes $(0.7$ to $1.3 \mathrm{~nm})$ grow. $^{21}$

The CoMoCAT process also involves the disproportionation of carbon monoxide gas, however the carbon nanotubes are grown on a special cobalt-molybdenum (CoMo) catalyst. ${ }^{22}$ In a high temperature $\left(700-950^{\circ} \mathrm{C}\right)$ chamber, the pure carbon monoxide gas is introduced at a total pressure that typically ranges from 1 to $10 \mathrm{~atm} .^{22}$ The CoMoCAT process generates a large amount of nanotube material, with over $80 \%$ of SWNTs grown by this method.

\subsection{Solubilization of Carbon Nanotube Bundles}

Carbon nanotubes form bundles due to the presence of strong intermolecular forces such as van der Waals and $\pi-\pi$ stacking, as shown in Figure $3{ }^{3}$ The entangled bundles are insoluble in all organic solvents and aqueous media. ${ }^{3}$ While it is possible to disperse carbon nanotubes in some solvents through vigorous sonication, they immediately aggregate and re-form bundles when the sonication process is momentarily halted. ${ }^{3}$ Due to their lack of solubility in any solvents, the use and applications of carbon nanotubes are generally limited. As previously discussed, commercial SWNT synthetic methods result in a complex mixture of semiconducting and metallic SWNTs, as well as amorphous carbon and metal catalyst particles. ${ }^{23-27}$ As a result, significant research toward the solubilization of carbon nanotubes is currently under investigation. Mainly, covalent and noncovalent functionalization methods have been extensively studied and employed for the solubilization of carbon nanotubes. ${ }^{28-30}$ 
M.Sc. Thesis - C. Shamshoom - McMaster University- Chemistry and Chemical Biology

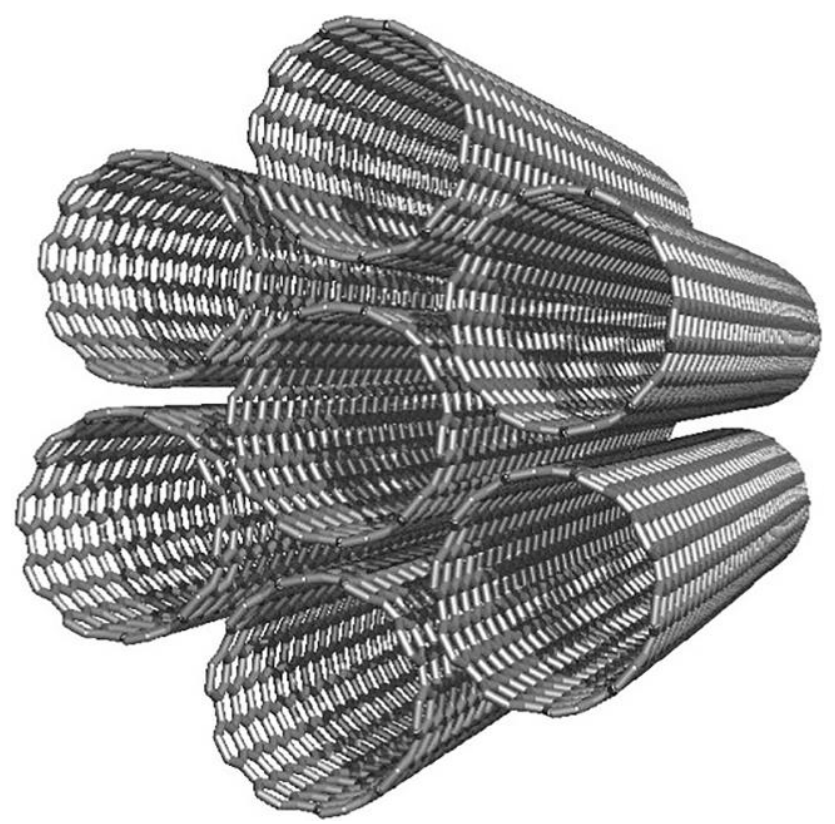

Figure 3. Idealized representation of SWNTs in a bundle. Reproduced with permission from [Hirsch, A. Angew. Chemie - Int. Ed. 2002, 41 (11), 1853-1859]. ${ }^{30}$ Copyright John Wiley \& Sons, 2002.

\subsubsection{Covalent Functionalization of SWNTs}

Some of the first accounts of covalent chemistry on SWNTs involve ultrasonication of a mixture of concentrated nitric and sulfuric acid to introduce oxygenated functional groups (such as carboxylic acids) at the ends and at defects on the sidewalls of carbon nanotubes. ${ }^{15}$ The resultant carboxyl groups enable further functionalization through amidation or esterification to introduce various groups onto the SWNTs. ${ }^{15}$ Covalent decoration of nanotube sidewalls has also been achieved using a variety of reactive reagents, such as carbenes, nitrenes, and radicals, which have enabled introduction of many different functionalities on the nanotube surface. ${ }^{3}$ These covalent approaches have been utilized for the solubilization of SWNTs in organic or aqueous media as well as their 
M.Sc. Thesis - C. Shamshoom - McMaster University- Chemistry and Chemical Biology

incorporation into polymer matrices. ${ }^{3,31}$ However, this method of solubilization presents some drawbacks as it alters the intrinsic electrical, mechanical and optical properties of SWNTs. Covalent modification utilizes reactive intermediates that react with the $\mathrm{sp}^{2}$ hybridized carbon atoms of the SWNT sidewall, resulting in $\mathrm{sp}^{3}$-carbon defects that damage their intrinsic properties. ${ }^{28}$ In addition, covalent chemistry on the surface of SWNTs can reduce their lengths into fragments that range from 100 to $300 \mathrm{~nm}$ by refluxing in nitric acid. ${ }^{15,32}$

\subsubsection{Non-Covalent Functionalization of SWNTs}

Non-covalent functionalization utilizes a dispersant that adsorbs onto the SWNT surface, forming a supramolecular complex that does not disrupt the nanotube's extended $\pi$-system. ${ }^{33,34}$ This method of functionalization is intriguing since it offers the potential of introducing a reactive handle whilst preserving the desirable electronic properties of SWNTs. ${ }^{3}$ A variety of dispersants can be employed for non-covalent functionalization of SWNTs including surfactants, ${ }^{35-37}$ aromatic small molecules,${ }^{38-40}$ conjugated polymers,${ }^{41-}$ 43 and biomacromolecules. ${ }^{10,44-49}$ In general, the dispersant molecules interact with the tubular surface of SWNTs through van der Waals or $\pi-\pi$ stacking to form a supramolecular complex that is controlled by thermodynamics. ${ }^{3}$

One of the first attempts to disperse carbon nanotubes employing a surfactant was achieved by O'Connell and coworkers in 2002. ${ }^{10}$ Their dispersion protocol involved vigorous sonication of a mixture of HiPCO SWNTs and the anionic surfactant sodium dodecyl sulfate (SDS) in $\mathrm{H}_{2} \mathrm{O}$. The mechanical separation of SWNT bundles through 
M.Sc. Thesis - C. Shamshoom - McMaster University- Chemistry and Chemical Biology

sonication was followed by the interaction of the hydrophobic tails of the surfactant molecules with the SWNT wall, as well as the hydrophilic heads, which create a shell around each individual tube. The resultant SWNT-SDS micelles prevented their reaggregation, generating a stable dispersion in $\mathrm{H}_{2} \mathrm{O}$. In addition to SDS, other surfactants such as sodium dodecylbenezenesulfonate (SDBS), sodium cholate (SCh), and deoxycholate (DOC) were employed in the dispersion of SWNTs (Figure 4), albeit with no selectivity for different species. ${ }^{50,51}$

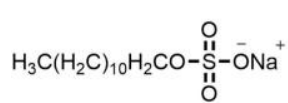

SDS

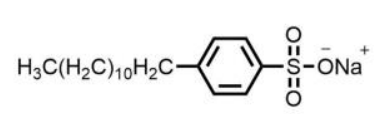

SDBS

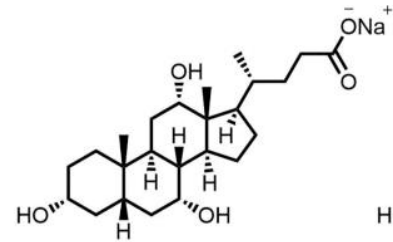

$\mathrm{sCh}$

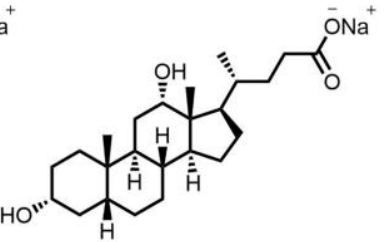

DOC

Figure 4. Structures of reported surfactants used in the individualization of SWNTs: sodium dodecyl sulfate (SDS), sodium dodecylbenezenesulfonate (SDBS), sodium cholate (SCh), and deoxycholate (DOC).

Another advancement in the processing of raw nanotubes appeared in 2006, when Arnold et al. reported the aqueous separation of SWNTs using density-gradient ultracentrifugation (DGU). ${ }^{52}$ This is a method traditionally used in biochemistry for separating biological macromolecules such as nucleic acids and proteins, based on their buoyant densities. ${ }^{53}$ In the study by Arnold et al., surfactant-coated SWNTs in aqueous media were subjected to ultracentrifugation $(\sim 200000 \mathrm{~g})$ in a mass density gradient. In response to the resulting centripetal force, the surfactant-coated SWNTs travel through the density gradient until the density of the medium is identical to their respective buoyant densities. ${ }^{52}$ At this point, the aqueous-suspended SWNTs are spatially separated, shown in 
M.Sc. Thesis - C. Shamshoom - McMaster University- Chemistry and Chemical Biology

Figure 5 as individually coloured bands that contain SWNTs of unique diameter and chirality. The SWNT species can be subsequently extracted employing simple fractionation techniques. Since the introduction of DGU for nanotube purification, numerous studies have been published utilizing this technique, ${ }^{54-56}$ in addition to other work which demonstrated that gel electrophoresis allows for separation of nanotubes by electronic type; i.e. semiconducting SWNTs (sc-SWNTs) and metallic SWNTs (m-SWNTs). ${ }^{57,58}$

Despite the progress made with these methods, only sub-microgram quantities of pure SWNT samples can be produced. This is mainly due to the relative inefficiency of these methods, as many iterations of the separation protocol are required for approximately one milligram of a pure SWNT sample. As a result, DGU and gel-based techniques produce costly SWNT samples, making them unsuitable for large-scale, commercial nanotube purification. $^{13}$ 
M.Sc. Thesis - C. Shamshoom - McMaster University- Chemistry and Chemical Biology

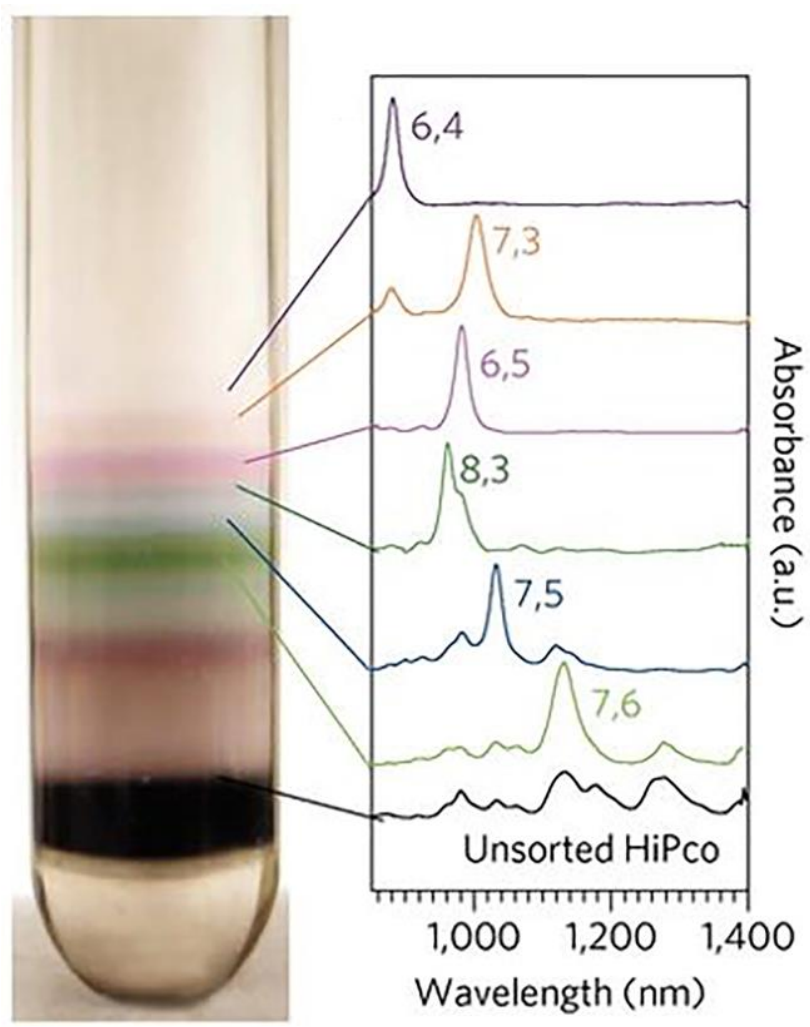

Figure 5. A photograph (left) of a centrifuge tube containing several bands of SWNTs sorted by the density gradient ultracentrifugation (DGU) technique, and their corresponding UV-Vis-NIR absorption spectra (right). Reproduced with permission from [Ghosh, S.; Bachilo, S. M.; Weisman, R. B. Nat. Nanotechnol. 2010, 5 (6), 443-450]. ${ }^{59}$ Copyright Nature Group, 2009.

DNA is another class of molecules employed in the dispersion of SWNTs. DNA molecules contain nucleotide bases that interact with the surface of SWNTs through $\pi-\pi$ stacking interactions, which allows for the DNA strands to helically wrap around SWNTs. ${ }^{60}$ In 2009, Zheng and co-workers used highly-specific DNA sequences for the separation and isolation of twelve sc-SWNT chirlaties as shown in Figure $6{ }^{61}$ 
M.Sc. Thesis - C. Shamshoom - McMaster University- Chemistry and Chemical Biology

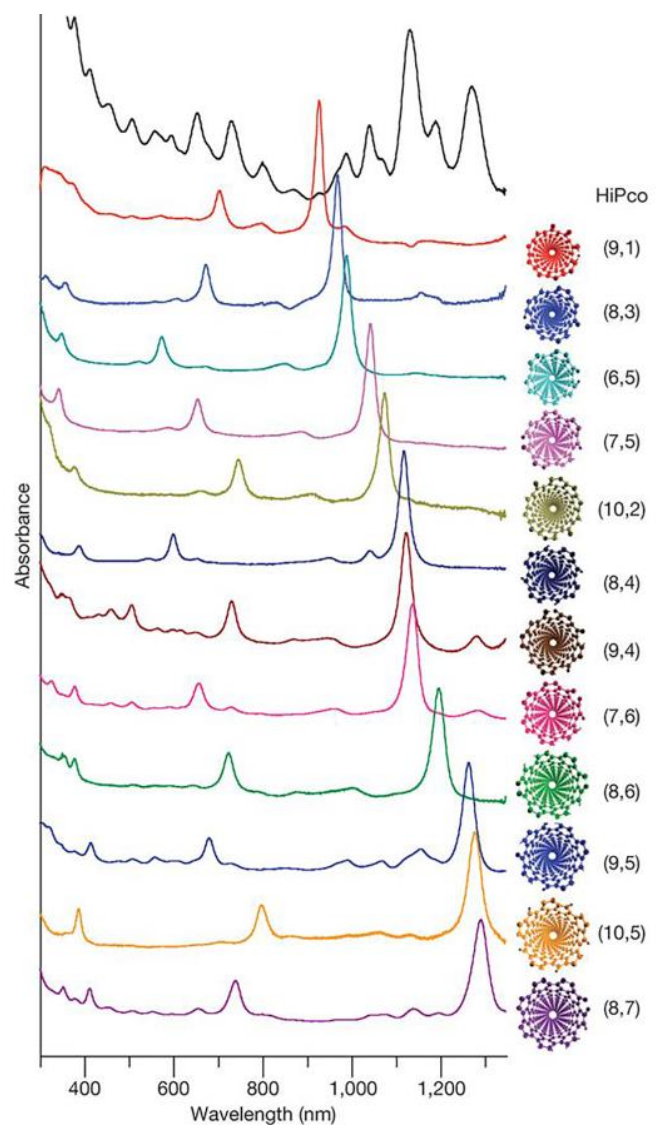

Figure 6. UV-Vis-NIR absorption spectra of 12 sc-SWNT species isolated via DNA and IEX and raw HiPCO SWNTs (top black spectrum). Reproduced with permission from [Tu, X.; Manohar, S.; Jagota, A.; Zheng, M. Nature 2009, 460 (7252), 250-253] ${ }^{61}$ Copyright Nature Publishing Group, 2009.

\subsubsection{Conjugated Polymers for Non-covalent Functionalization of SWNTs}

Compared to the techniques discussed in Section 1.2.2, conjugated polymers are a viable alternative as they offer a scalable and time efficient method for the non-covalent functionalization of SWNTs. ${ }^{34}$ Over the past decade, conjugated polymers have been employed for solubilization of carbon nanotubes, and recently have been shown to generate dispersions selective for different SWNT species. Carbon nanotubes are dispersed through 
M.Sc. Thesis - C. Shamshoom - McMaster University- Chemistry and Chemical Biology

$\pi$-stacking interactions between the backbone of the $\pi$-conjugated polymer with the SWNT surface. The resultant non-covalent interaction disrupts the intrinsic van der Waals forces in nanotube bundles and prevents their reaggregation. ${ }^{34}$ Depending on the flexibility of the polymer backbone, they can either warp helically or simply adsorb onto the nanotube surface to generate polymer-SWNT complexes. This method leads to enhanced dispersions of SWNTs using various conjugated polymers. ${ }^{62-64}$ Some of the most studied conjugated polymer structures include polyfluorene, polycarbazole, polythiophene, poly(phenylene vinylene), and poly(phenylene ethynylene)s. ${ }^{65}$ Polyfluorenes and their copolymers have been heavily investigated for the electronic separation of SWNTs. They are particularly interesting due to their ease of synthesis and functionalization. Specifically, polyfluorenes have been shown to selectively disperse semiconducting SWNT species. ${ }^{13}$ However, there are numerous factors that affect the selectivity in polymer-SWNT interactions such as the polymer backbone and molecular weight, the type of side chains present, as well as the solvents employed for dispersions. ${ }^{66}$

\section{Characterization of SWNTs}

Characterization of SWNTs can be achieved through various spectroscopic techniques such as absorbance, photoluminescence (PL), Raman, Rayleigh, and magnetooptical spectroscopy. ${ }^{67}$ Absorbance, PL and Raman spectroscopy have become particularly attractive for characterization of SWNTs because they involve rapid, facile sample preparation and cause minimal damage to nanotube samples. ${ }^{67}$ The various photophysical processes involved in each spectroscopic method can be described by the electronic density 
M.Sc. Thesis - C. Shamshoom - McMaster University- Chemistry and Chemical Biology

of states (DOS) diagram of a SWNT, as shown in Figure 7. The absorption process occurs when an incident photon that matches the corresponding excitonic energy difference $\left(\mathrm{E}_{\mathrm{ii}}\right)$, generates an exciton (photoexcited electron and a hole), which is represented by the upward arrow in Figure 7a. The way in which the exciton recombines results in other photophysical processes including Rayleigh scattering, photoluminescence, and Stokes Raman. ${ }^{67}$ As shown in Figure 7b, Rayleigh scattering is an elastic process which occurs upon emission of light without the exciton gaining or losing energy. Photoluminescence (Figure 7c) results from excitonic relaxion to the lowest lying excited state $\left(c_{1}\right)$, followed by recombination to emit a lower energy photon $\left(E_{11}\right)$, compared to the incident photon. In addition, the exciton may lose or gain energy via emission or absorption of a phonon in the scattering process to an intermediate state, resulting in recombination and emission of a scattered photon with slightly lower $\left(\mathrm{E}_{22}-\mathrm{E}_{p h}\right)$ or higher $\left(\mathrm{E}_{22}+\mathrm{E}_{p h}\right)$ energy than the incident photon. These processes are inelastic and are referred to as Stokes (Figure 7d) and Anti-Stokes Raman, respectively. ${ }^{67}$ 
(a)

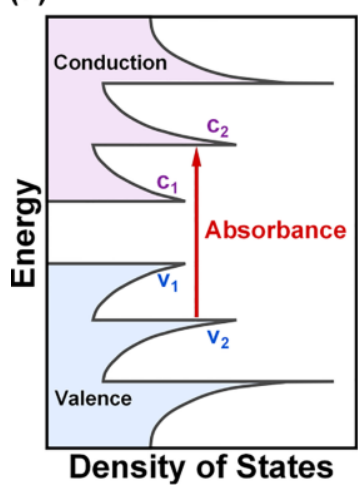

(b)

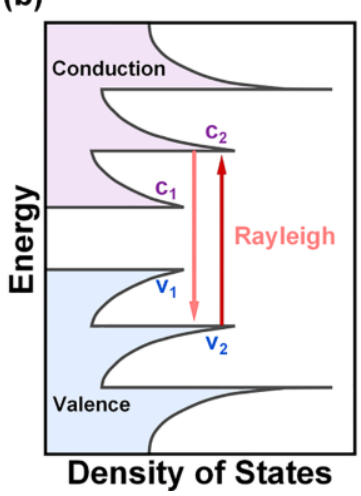

(c)

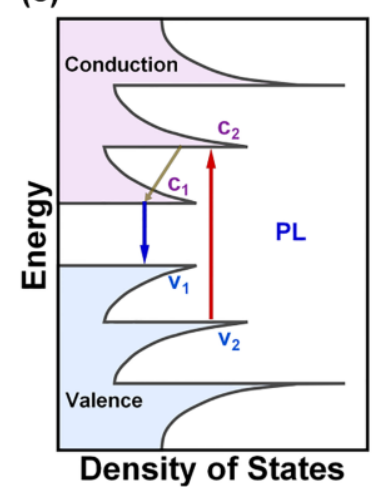

(d)

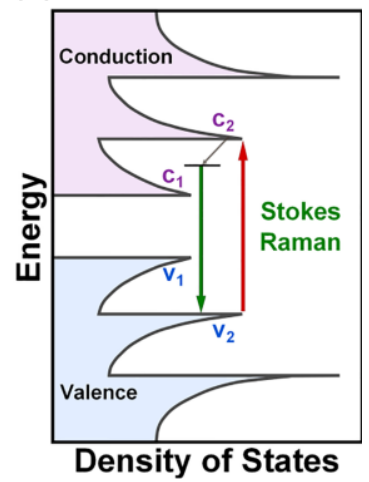

Figure 7. Schematic representation of the various optical processes of SWNTs, such as (a) absorbance, (b) Rayleigh scattering, (c) photoluminescence (PL), and (d) Stokes Raman processes. Reproduced with permission from [Rice, N. A. Separation of Single-Walled Carbon Nanotubes By Electronic Type Using Conjugated Polymers, McMaster University, 2015]. ${ }^{13}$ Copyright 2015.

\subsubsection{Absorption spectroscopy}

Absorption spectroscopy is a fundamental, experimentally facile optical measurement for characterization of SWNTs. If the optical features of a nanotube sample are well defined, absorption spectroscopy can provide information on the $(n, m)$ chirality distribution of a SWNT sample, ${ }^{68}$ as well as the ability of a separation protocol to achieve electronic purity. In 1999, Kataura et al. obtained one of the first absorption spectra of SWNTs in thin film samples that were prepared from a dispersion of SWNTs in ethanol. ${ }^{12}$ Within the measured spectral range (200 to $2500 \mathrm{~nm}$ ), only three broad absorbance bands were observed, with no fine features present in the spectrum. Later, in 2002, O'Connell and coworkers obtained an absorption spectrum of SWNTs dispersed with sodium dodecyl sulfate (SDS) in $\mathrm{H}_{2} \mathrm{O}$, where they observed fine features corresponding to various SWNT species present in the sample. ${ }^{69}$ Their dispersion protocol involved vigorous sonication of the SDS and SWNTs mixture to detach nanotubes from a bundle and allow for the SDS to 
M.Sc. Thesis - C. Shamshoom - McMaster University- Chemistry and Chemical Biology

coat nanotubes in order to prevent reaggregation. This was followed by a centrifugation step, which removes any bundles remaining in solution. ${ }^{70}$ The individualization of SWNTs in aqueous micellar suspensions resulted in an optical absorption spectrum that contains numerous sharp peaks corresponding to various optical transitions of sc-SWNTs and mSWNTs in solution. The absorption features of carbon nanotubes arise from the electronic transitions from the valence to conduction bands of the van Hove singularities, where the observed absorption features depend on nanotube diameter and chirality. ${ }^{70,71}$ For small diameter HiPCO (0.7 to $1.2 \mathrm{~nm})$ SWNTs, the absorbance features arising from metallic SWNTs is found at approximately 440 to $650 \mathrm{~nm}\left(\mathrm{M}_{11}\right)$ while the semiconducting region is located roughly between 570 to $850 \mathrm{~nm}\left(\mathrm{~S}_{22}\right)$ and 850 to $1600 \mathrm{~nm}\left(\mathrm{~S}_{11}\right){ }^{70,71}$

It is therefore possible to assess the electronic nature of a SWNT dispersion considering that the metallic and semiconducting absorptions occur at different regions within the UV-Vis-NIR spectral range. In addition, semi-quantitative information can be collected using absorption spectroscopy by integration of the area under the peaks of metallic and semiconducting regions. However, it is difficult to precisely determine boundaries for integration areas due to the overlap between several spectral excitation regions. Also, the overlap of transitions from numerous SWNT species results in uncertainty when making specific $(n, m)$ assignments of a heterogenous sample. ${ }^{72}$ Other techniques, such photoluminescence (PL) mapping and Raman spectroscopy are therefore required to obtain a thorough understanding of the sample properties. 
M.Sc. Thesis - C. Shamshoom - McMaster University- Chemistry and Chemical Biology

\subsubsection{Photoluminescence (PL) Mapping}

Photoluminescence (PL) Mapping is a spectroscopic technique used to characterize sc-SWNTs, which possess a bandgap and therefore are capable of fluorescence. It measures the fluorescence intensity of sc-SWNTs with respect to multiple excitation and emission wavelengths. ${ }^{13} \mathrm{PL}$ mapping is a powerful tool for investigation of SWNT dispersions because it offers sensitive qualitative information regarding the presence of specific semiconducting nanotube species. However, the SWNTs in solution must be effectively debundled with limited presence of metallic SWNT species, as such factors can dramatically reduce the observed PL intensity. ${ }^{73}$

The 2D PL map of individualized SWNTs contains numerous peak maxima that can be assigned $(n, m)$ values, using data published in the literature. ${ }^{74}$ Utilising a tightbinding approximation, it is possible to relate chirality assignments to transition energies and diameters of carbon nanotubes. ${ }^{75}$ However, it is difficult to obtain a direct comparison between theoretical and experimental results due to the complexity of the real nanotube as opposed to the suggested approximation. This complexity arises from extrinsic environmental factors such as the presence of other nanotubes, solvents, as well as dispersants, and typically results in a red-shift in the experimentally obtained emission maxima, in addition to broadening of optical features. ${ }^{76}$ Nevertheless, it is possible to assign $(n, m)$ values for a given SWNT sample by choosing literature data that was acquired under identical or similar environmental conditions as the sample being investigated. 
M.Sc. Thesis - C. Shamshoom - McMaster University- Chemistry and Chemical Biology

\subsubsection{Resonant Raman Spectroscopy}

Raman spectroscopy measures the scattered photon intensity with respect to the Raman shift, which is described as the frequency difference $\left(\mathrm{cm}^{-1}\right)$ between the incident and the scattered photon. ${ }^{67}$ It is a versatile and non-destructive technique utilized in probing various $\mathrm{sp}^{2}$-hybridized carbonaceous materials including metallic and semiconducting SWNTs. In particular, Raman spectroscopy is useful in determining the $(n, m)$ distributions ${ }^{77}$ as well as characterizing the electronic purity and defect density for both mSWNTs and sc-SWNTs. ${ }^{78}$ In resonance Raman spectroscopy, the incident or scattered photon is at a frequency near the frequency of an electronic transition between the valence and conduction bands of the SWNT. This provides enough energy to excite the electrons to a higher electronic state, resulting in a very large Raman signal. Therefore, resonant Raman spectroscopy offers enhanced signals which allows for detection of SWNTs on a single nanotube level. ${ }^{79}$ Figure 8 depicts a plot of electronic transition energies as a function of nanotube diameter and type, generally referred to as a Kataura plot. The horizontal lines depict the excitation wavelengths typically used to study carbon nanotubes, where different excitation wavelengths are in resonance with different SWNT types. This plot shows that multiple excitation wavelengths are required in order to probe the majority of nanotube species within a sample. 
M.Sc. Thesis - C. Shamshoom - McMaster University- Chemistry and Chemical Biology

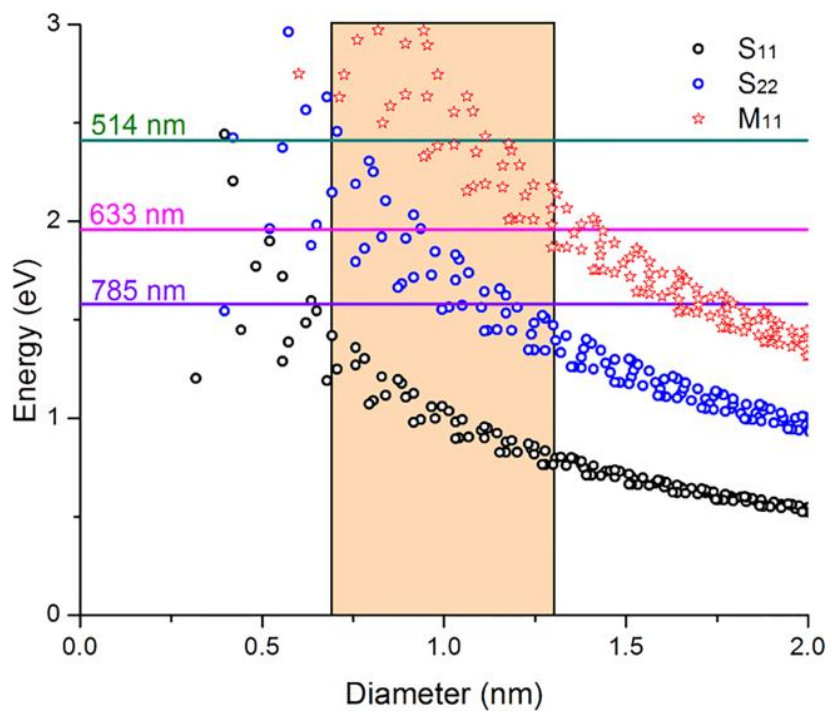

Figure 8. Semiempirical Kataura plot of $S_{11}$ (black), $S_{22}$ (blue) and $M_{11}$ (red). The electronic transitions data for sc-SWNT ${ }^{74}$ and $\mathrm{m}-\mathrm{SWNT}{ }^{80,81}$ were obtained from literature sources. The orange box depicts the expected diameter range for HiPCO SWNTs (diameter range from $\sim 0.7$ to $1.3 \mathrm{~nm}$ ). The horizontal lines denote the three laser excitation wavelengths $(514,633$, and $785 \mathrm{~nm})$ used for Raman studies in this thesis. Reproduced with permission from [Rice, N. A. Separation of Single-Walled Carbon Nanotubes By Electronic Type Using Conjugated Polymers, McMaster University, 2015]. ${ }^{13}$ Copyright 2015.

One of the first accounts of resonant Raman experiments on carbon nanotubes was reported by Rao and co-workers in $1997 .{ }^{82}$ These experiments were conducted on SWNT powders prepared by laser ablation, and revealed four prominent peaks in the Raman spectrum. ${ }^{82}$ Figure 9 shows a typical Raman spectrum for HiPCO SWNTs with the four observed regions, which were later identified as follows: the radial breathing mode (RBM) from $\sim 100$ to $400 \mathrm{~cm}^{-1}$, the D-band from $\sim 1250$ to $1450 \mathrm{~cm}^{-1}$, the G-band from $\sim 1550$ to $1595 \mathrm{~cm}^{-1}$, and the $\mathrm{G}^{\prime}$ (or $\mathrm{D}^{*}$ ) mode at $\sim 2500$ to $2900 \mathrm{~cm}^{-1} .83$ 


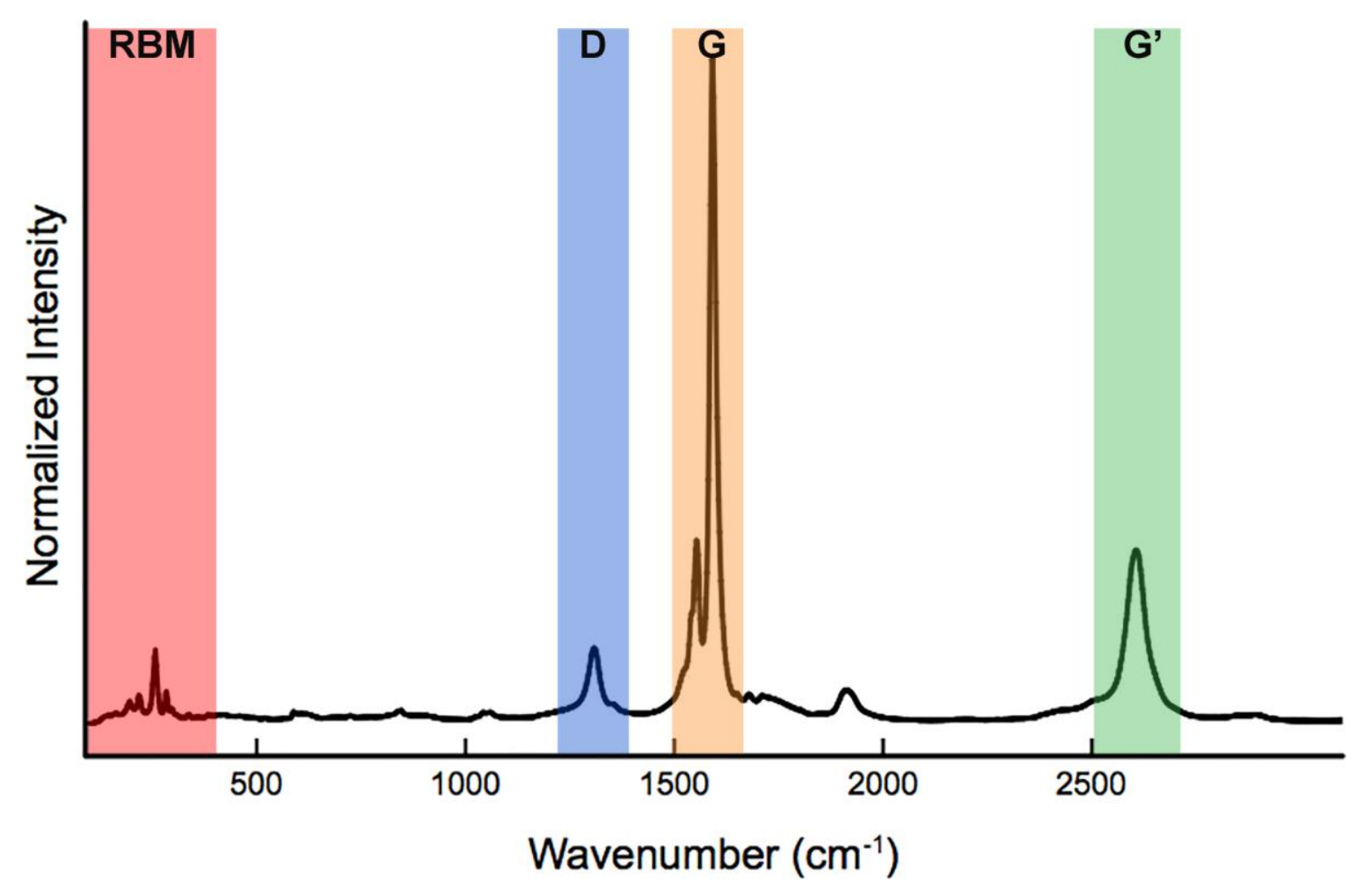

Figure 9. A typical Raman spectrum for a SWNT sample, showing the RBM (red), D-band (blue), the G-band (orange), and G'-band (green) regions.

The four regions shown in Figure 9 provide useful information for the characterization of SWNTs. The RBM region results from the out-of-plane bond stretching of all the carbon atoms in the radial direction, making the RBM mode unique to carbon nanotubes. ${ }^{83}$ Thus, the observation of the RBM region in the Raman spectrum is indicative of the presence of SWNTs in a sample.

The D-band, also known as the disorder mode, is found in the Raman spectra of various carbonaceous materials. The disorder mode arises from elastic scattering of electrons by structural defects including vacancies, impurities, or $\mathrm{sp}^{3}$-hybridized carbon atoms. Thus, the D-band feature is utilized in the determination of the chemical purity of the carbon nanotubes. ${ }^{84,85}$ 
M.Sc. Thesis - C. Shamshoom - McMaster University- Chemistry and Chemical Biology

The G-band mode (also known as the tangential mode) is a Raman feature present in graphitic structures containing $\mathrm{sp}^{2}$-hybridized carbon atoms, including carbon nanotubes. It results from the in-plane carbon-carbon bond stretches in graphitic materials. The G-band consists of two peaks: A lower frequency $\mathrm{G}^{-}$and a higher frequency $\mathrm{G}^{+}$. For sc-SWNTs, both the $\mathrm{G}^{-}$and $\mathrm{G}^{+}$have Lorentzian line shapes, while for $\mathrm{m}-\mathrm{SWNTs}$ the $\mathrm{G}^{-}$ exhibits a broader Breit-Wigner-Fano (BWF) line shape. ${ }^{86}$ Depending on the shape of the $\mathrm{G}^{-}$mode, it is possible to distinguish between $\mathrm{m}-\mathrm{SWNT}$ and sc-SWNTs in a given sample. Raman spectra lacking the BWF line shape in the G-band indicate an absence of metallic SWNTs in the sample. The $\mathrm{G}^{\prime}$-band (or $\mathrm{D}^{*}$ ) is a disorder based overtone of the D-mode that is not unique to SWNTs and is found in the Raman spectra of other carbonaceous materials. $^{83}$

\subsection{Introduction to Supramolecular Chemistry}

The emerging field of modern supramolecular chemistry continues to grow from its humble origins in the early 1960's with the pioneering contributions of Lehn, Cram and Pedersen that were subsequently awarded the Nobel prize in $1987 .{ }^{87-89}$ In his Nobel Prize lecture, Lehn describes supramolecular chemistry as "chemistry beyond the molecule," 87 occurring when two or more chemical species associate through intermolecular forces such as electrostatic interactions, hydrogen bonding, $\pi-\pi$ interactions, or van der Waals interactions. $^{87}$

Supramolecular Chemistry is the study of a system composed of molecules or ions which are held together by non-covalent interactions..$^{90}$ This field of study may be divided into two distinct categories: i) host-guest chemistry and ii) molecular self-assembly. Host- 
M.Sc. Thesis - C. Shamshoom - McMaster University- Chemistry and Chemical Biology

guest chemistry involves the non-covalent interactions of a larger (host) molecule enclosing a smaller (guest) molecule to form a supramolecular complex. Meanwhile, selfassembly occurs due to the spontaneous, yet reversible association of two species to form a larger supramolecular complex..$^{90}$

\subsubsection{Host-Guest Chemistry}

Host-guest chemistry, which is a branch of supramolecular chemistry, explores molecular recognition that enables a guest molecule to bind to the cavity of a host to produce a "host-guest" complex. The host is typically a large molecule that contains a central hole or cavity, such as a synthetic cyclic compound or an enzyme. On the other hand, the guests can vary from small anions and cations, to larger molecules such as linear alkanes or complex hormones. ${ }^{90}$ Various host structures have been investigated, including cyclodextrins, ${ }^{91-93}$ calixarenes, ${ }^{94-96}$ cucurbiturils, ${ }^{97-99}$ and pillararenes. ${ }^{100-102}$

\subsubsection{Introduction to Pillar[5]arenes}

Pillar[5]arenes are macrocyclic molecules belonging to the family of paracyclophanes. Owing to the seminal work by Ogoshi and co-workers, ${ }^{103}$ the host-guest chemistry of pillar[5]arenes has attracted significant attention, primarily due to their simple one-step synthesis. Pillar[5]arenes are a class of macrocycles composed of hydroquinone units connected by methylene bridges at the para-positions, forming a "pillar-like" structure. The electron-rich dialkoxybenzene moieties of pillar[5]arenes form stable inclusion complexes with various electron-poor molecules, including viologens, ${ }^{104-106}$ 
M.Sc. Thesis - C. Shamshoom - McMaster University- Chemistry and Chemical Biology

alkanediamines, ${ }^{107,108}$ and alkylnitriles. ${ }^{109-111}$ Host-guest interactions with pillar[5]arenes have been used in polymers, ${ }^{112-114}$ sensors,,${ }^{108,115,116}$ and organogels. ${ }^{104,117,118}$

\subsubsection{First Appearance of Paracyclophanes}

The first reported synthesis of paracyclophanes was described by Gribble and Nutaitis in $1985 .^{119}$ The synthesis was discovered in an attempt to selectively reduce 4(hydroxymethyl)diphenyl methanol in the presence of sodium borohydride $\left(\mathrm{NaBH}_{4}\right)$ and trifluoroacetic acid (TFA), as shown in Scheme 2. Rather than the expected product, 4(hydroxymethyl)diphenylmethane, two other compounds were observed. Utilising ${ }^{1} \mathrm{H}$ NMR spectroscopy and mass spectrometry, it was apparent that the mixture of products was composed of the novel paracyclophanes $\mathbf{1}$ and $\mathbf{3}$. It was hypothesized that these novel paracyclophanes resulted from a Friedel-Crafts cyclo-oligomerization of the initially formed 5. It was later confirmed that TFA promoted the Friedel-Crafts alkylation of arenes with benzyl (or related) alcohols. ${ }^{119}$ 


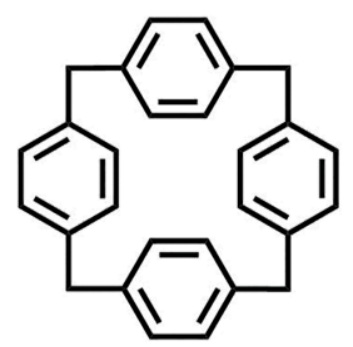

[1.1.1.1]paracyclophane

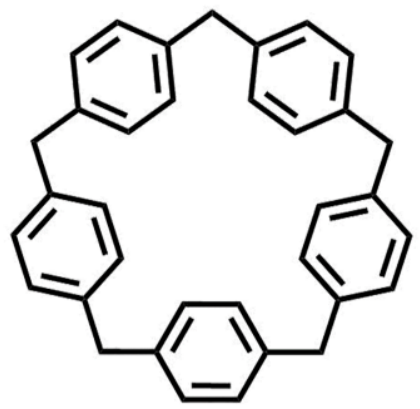

[1.1.1.1.1]paracyclophane 2

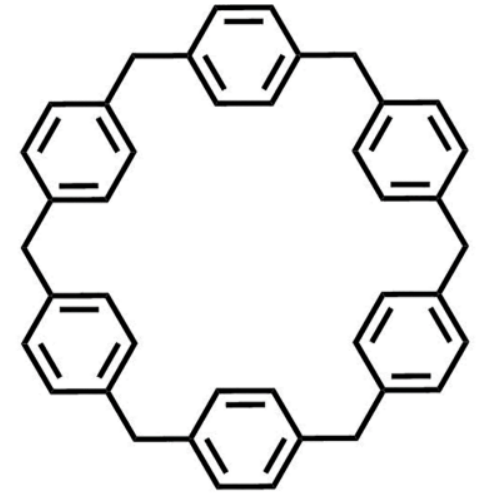

[1.1.1.1.1]paracyclophane 3

Scheme 1. Structures of various paracyclophanes.<smiles>OCc1ccc(C(O)c2ccccc2)cc1</smiles>

4

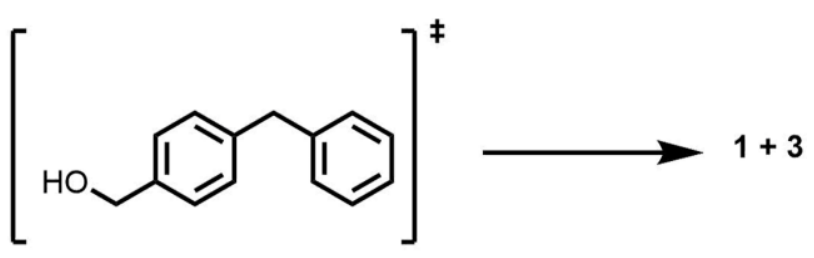

5

Scheme 2. The macrocycles obtained during the selective reduction of 4 .

Given the findings of their reaction, Gribble and Nutaitis sought to further investigate the Friedel-Crafts cyclo-oligomerization of various benzyl alcohol derivatives. As shown in Scheme 3, they began with commercially available 4-bromobenzophenone (6) to obtain the precursors 7,8 , and $\mathbf{9}$ utilizing a repetitive three-step homologation method. The conversion of alcohols 7,8 and 9 to their corresponding paracyclophanes involved reflux in the presence of TFA. While this resulted in low yields of $\mathbf{2}$ and $\mathbf{3}$, the formation of the cyclotetramer 1 was not observed. Regardless of further optimization, the macrocyclization step still generated low yields of $\mathbf{2}$ and $\mathbf{3}$. 


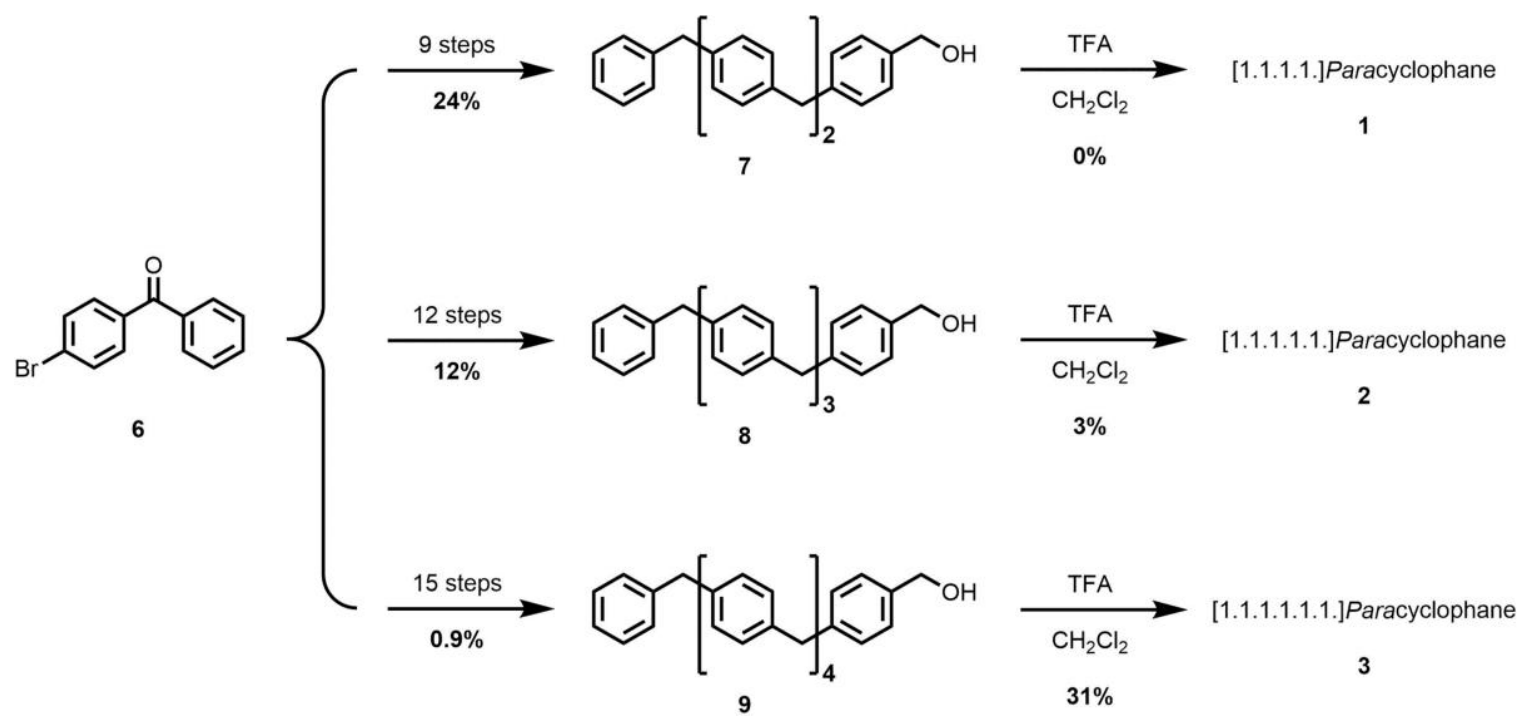

Scheme 3. Multi-step synthesis of paracyclophanes 1, 2, and 3.

Despite the fact that the chemistry of other cavitands, such as calixarenes, was popular due to their one-step, high-yielding synthesis and their ease of functionalization, ${ }^{120-122}$ the chemistry of pararcyclophanes did not receive much attention due to their multi-step, low yielding synthesis.

\subsubsection{First Efficient Synthesis of Pillar[n]arenes}

In 2008, Tomoki Ogoshi published an efficient synthesis for hydroquinolic derivatives connected by methylene bridges at the para positions (Scheme 4). ${ }^{103}$ Owing to their pillar-like structure, Ogoshi named these macrocyclic structures "pillar[n]arenes", where $n$ corresponds to the number of aromatic repeat units. The efficient, one-step synthesis of the cyclopentamer $(n=5)$ involved the reaction of commercially available 1,4 dimethoxybenzene with paraformaldehyde in the presence of a Lewis acid catalyst in dichloromethane and 1,2-dichloroethane. Under these reaction conditions, they obtained 
M.Sc. Thesis - C. Shamshoom - McMaster University- Chemistry and Chemical Biology

exclusively pillar[5]arene in $22 \%$ yield. ${ }^{103}$ The yield was improved to $71 \%$ upon reaction of one equivalent of the 1,4-dimethoxybenzene with three equivalents of paraformaldehyde. As a result of their facile and high-yielding synthesis from commercially available starting materials, the chemistry of pillar[5]arenes received significant attention within the scientific community.

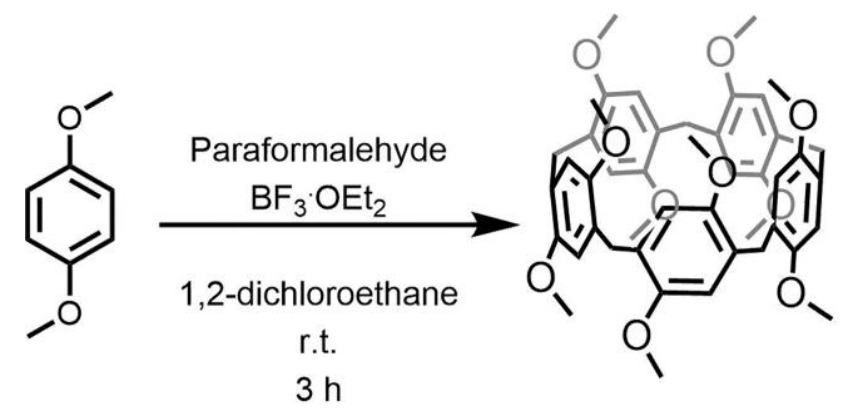

$22 \%$

Scheme 4. First efficient synthesis of pillar[5]arene reported by Ogoshi in 2008.

\subsubsection{Synthesis of Pillar[5]arenes under Thermodynamic Control}

The formation of a macrocycle is a generally low-yielding reaction where formation

of linear oligomers and polymers generally predominates. ${ }^{123,124}$ As a result, high-dilution techniques are employed to facilitate cyclization, hence increasing the overall yield of macrocycle formation. ${ }^{124}$ In 2011 , Nierengarten and co-workers reported that the highyielding synthesis of pillar[5]arenes occurs under thermodynamic control. ${ }^{125}$ They found that the cyclo-oligomerization between 2,5-bis(bromomethyl)-1,4-dialkoxybenzene and 1,4-dialkoxybenzene did not generate the expected cyclohexamer. Rather, they observed solely the formation of pillar[5]arene. Thermodynamic control is possible due to the reversibility of the Friedel Crafts reaction. As the formation of the cyclization products is 
M.Sc. Thesis - C. Shamshoom - McMaster University- Chemistry and Chemical Biology

carried out under thermodynamic control, their distribution depends on the relative stabilities of the final products (Figure 10). Nierengarten and co-workers concluded that the pillar[5]arene derivative is the thermodynamic product. They reported X-ray diffraction data for the product, which showed that the 1,4-dimethoxypillar[5]arene methylene bridge bond angle is $111.3^{\circ}$. This value is in accordance with the bond angle of $\mathrm{sp}^{3}$-hybridized carbons $\left(109.5^{\circ}\right)$ and the theoretical internal bond angle $\left(108^{\circ}\right)$ for a regular, unstrained pentagon. $^{126}$

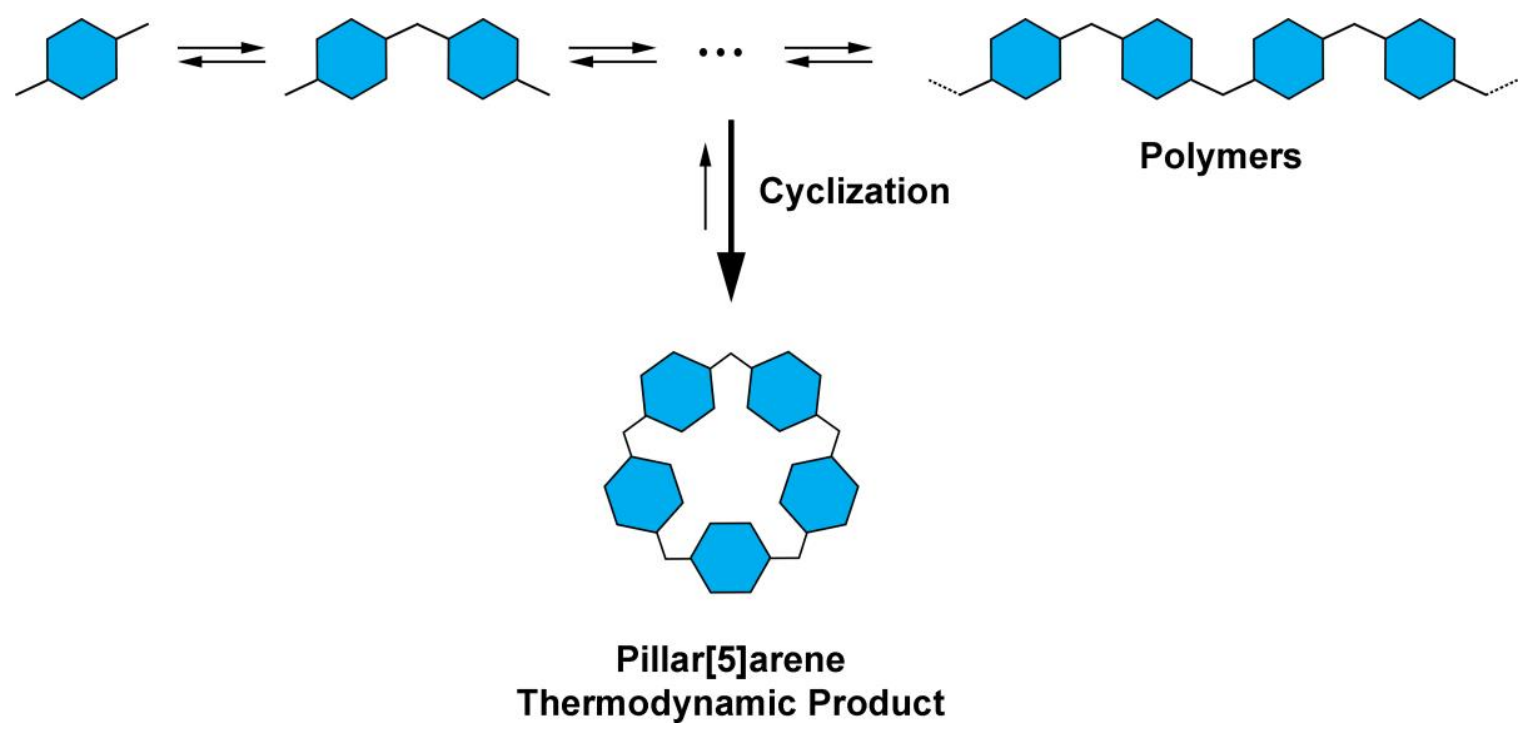

Figure 10. Schematic representation for the proposed pathway of pillar[5]arene under thermodynamic control.

\subsubsection{Mono-functionalization of Pillar[5]arenes}

In order to introduce additional functionality to pillar[5]arenes, they must be made reactive via incorporation of side chains containing alkyl bromide, alkyl azide, or alcohol moieties. Scheme 5 shows two reported approaches for the mono-functionalization of pillar[5]arenes that do not alter their host-guest properties. ${ }^{127}$ The first method involves the 
M.Sc. Thesis - C. Shamshoom - McMaster University- Chemistry and Chemical Biology

co-cyclization of two distinct monomers where one contains the desired reactive handle. In 2011, Stoddart and co-workers demonstrated that it is possible to prepare a copillar[5]arene by reaction of 1,4-dimethoxybenzene and 1-(2-bromoethoxy)-4methoxybenzene in the presence of paraformaldehyde and $\mathrm{BF}_{3} \cdot \mathrm{OEt}_{2} .{ }^{108}$ The resultant mono-azide pillar[5]arene can undergo copper-mediated azide-alkyne cycloaddition (CuAAC) with a terminal alkyne to prepare a pillar[5] arene derivative. ${ }^{108,128-130}$

The second strategy for mono-functionalization of pillar[5]arenes was employed by Ogoshi and co-workers in 2011. ${ }^{131}$ They achieved the mono-demethylation of dimethoxy pillar[5]arene using less than one equivalent of $\mathrm{BBr}_{3}$ and isolated the product in $22 \%$ yield. The reaction was later optimized by Cao and coworkers, utilising four equivalents of $\mathrm{BBr}_{3}$ with freshly distilled chloroform, and stirred at $-5{ }^{\circ} \mathrm{C}$ to obtain a $60 \%$ yield of the mono-functionalized pillar[5]arene. ${ }^{131}$ The pillar[5]arene bearing a single hydroxyl group, can be further functionalized under Williamson ether synthesis conditions to generate novel pillar[5]arene derivatives. ${ }^{132,133}$ 
M.Sc. Thesis - C. Shamshoom - McMaster University- Chemistry and Chemical Biology

\section{Approach 1: Co-cyclization}

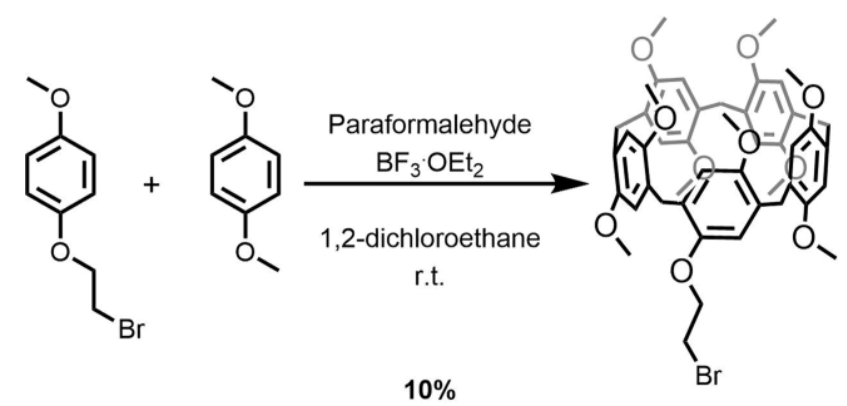

\section{Approach 2: Deprotection}

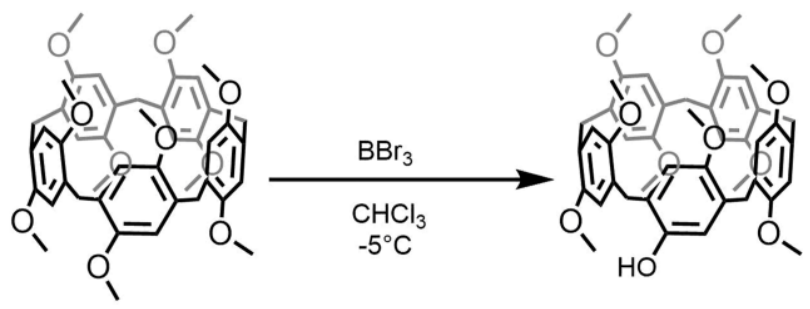

$60 \%$

Scheme 5. Two approaches for the mono-functionalization of pillar[5]arenes.

\subsubsection{Host-guest Properties of Pillar[5]arenes}

Pillar[5]arenes are host molecules with symmetrical central cavities that are approximately $5 \AA$ in diameter. ${ }^{134}$ The inner cavity of pillar[5]arene is electron rich due to the electron-donating alkoxy moieties located at the rim. In addition, the pillar-like structure enhances their $\pi$-electron density, making pillar[5]arenes excellent hosts for guests containing cationic or electron-withdrawing groups. ${ }^{134}$ There are several reports within the literature demonstrating that pillar[5]arenes form inclusion complexes with aromatic compounds like pyridinium and viologen derivatives, ${ }^{105,135}$ as well as linear 
M.Sc. Thesis - C. Shamshoom - McMaster University- Chemistry and Chemical Biology

alkanes containing electron-withdrawing amine, ${ }^{136}$ ammonium, ${ }^{136}$ cyano, ${ }^{137}$ and halogen ${ }^{138}$ end groups.

Supramolecular titration is a common method utilized in quantitative analysis of the intermolecular interactions between a host (such as pillar[5]arenes) and a guest molecule. It involves gradual addition of one component (typically the guest) to the system (host) while monitoring a physical property such as a specific chemical resonance (NMR spectroscopy) or absorption band (UV-Vis spectroscopy) that is sensitive to the supramolecular interaction(s) of interest. ${ }^{139}$ The acquired spectroscopic data is then compared and fitted to a binding model to obtain information about the system, such as the association constant $\left(K_{a}\right) .{ }^{139}$ Host-guest interactions formed with pillar[5]arenes have found applications in materials such as polymers, ${ }^{112-114}$ sensors, ${ }^{108,115,116}$ and organogels. ${ }^{104,117,118}$

\subsection{Objectives}

To date, most structural modifications have aimed to achieve enriched dispersions of semiconducting SWNTs, with recent efforts aimed toward developing polymer backbone structures capable of conformational changes or depolymerization in response to a stimulus. ${ }^{140-144}$

Recently, we have prepared polyfluorene derivatives possessing azide groups in their side chains, and then used these derivatives to noncovalently functionalize SWNTs. ${ }^{145-147}$ We have demonstrated that it is possible to decorate the resulting polymerSWNT complex using the Strain-Promoted Azide-Alkyne Cycloaddition (SPAAC) ${ }^{147}$ or 
M.Sc. Thesis - C. Shamshoom - McMaster University- Chemistry and Chemical Biology

the Copper-Mediated Azide-Alkyne Cycloaddition (CuAAC). ${ }^{146}$ These results indicate that conjugated polymer side chains can be tuned to impart interesting characteristics to the polymer-SWNT complex. The aim of this project is to utilize the same design principle to produce polymer-SWNT complexes that contain macrocycles in the polymer side chains to influence the dispersion properties of the resulting polymer-SWNT complex.

Macrocycles have received intense interest in the literature, ${ }^{148-153}$ and encompass several key scaffolds that include cyclodextrins, ${ }^{91-93}$ calixarenes, ${ }^{95,96,154}$ cucurbiturils, ${ }^{97-99}$ and pillararenes. ${ }^{100,155,156}$ Owing to the seminal work by Ogoshi and co-workers, ${ }^{103}$ pillar[5]arenes have attracted significant attention due to their facile one-step synthesis and their unique structure, composed of hydroquinone units connected by methylene bridges at the para-positions that form a "pillar-like" structure. These pillar[5]arenes can be further derivatized to install a single functional handle that can be used in subsequent functionalization. The electron-rich dialkoxybenzene moieties of pillar[5]arenes form stable inclusion complexes with various electron-poor molecules, including viologens, ${ }^{104-}$ 106 alkanediamines, ${ }^{107,108}$ and alkylnitriles. ${ }^{109-111}$ Host-guest interactions with pillar[5] arenes have been used in polymers, ${ }^{112-114}$ sensors,,${ }^{108,115,116}$ and organogels. ${ }^{104,117,157}$ The aim of this study is to explore the incorporation of pillar[5] arenes onto the surface of a polymer-SWNT complex. The first main objective is to characterize the dispersion properties before and after macrocycle incorporation using UV-Vis-NIR, Raman, and fluorescence spectroscopy, and determine the SWNT concentration in solution using thermogravimetric analysis (TGA). The second objective is to incorporate the cavitandcontaining polymer-SWNT dispersion into an organogel using a bis(alkylnitrile)- 
M.Sc. Thesis - C. Shamshoom - McMaster University- Chemistry and Chemical Biology

functionalized polymer as a crosslinking agent. The final goal of this project involves characterization of the organogel before and after incorporation of SWNTs by determining their Young's Modulus. 
M.Sc. Thesis - C. Shamshoom - McMaster University- Chemistry and Chemical Biology

\section{Chapter 2: Preparation and Characterization of Polymer- SWNT Complexes}

\subsection{Introduction}

Chapter 2 focuses on the synthesis and functionalization of polyfluorene to generate a pillar[5] arene-functionalized conjugated polymer. The synthetic approach involved preparation of a polyfluorene that contains alkyl bromides in the side chain that can be coupled with a mono-hydroxylated pillar[5]arene in the presence of a base and a phase transfer catalyst. The alkyl bromides of the polyfluorene serve as reactive handles for further functionalization of the polymer, such that the nucleophilic pillar[5]arene derivative attacks the electrophilic carbon of the alkyl bromide side chains to generate the macrocycle-functionalized polymer. Also, the preparation and characterization of the polymer-SWNT dispersions are discussed in this chapter. In addition, the host-guest properties of the polymer-SWNT dispersion are explored using ${ }^{1} \mathrm{H}-\mathrm{NMR}$ spectroscopy.

\subsection{Polymer Synthesis}

To prepare the macrocycle-containing polymer-SWNT complex, we first synthesized a polyfluorene derivative containing alkyl bromides (PF-Br) according to literature procedures (see Appendix B: Synthetic Procedures for details). ${ }^{158}$ GPC analysis of PF-Br revealed that the polymer had a number average molecular weight $\left(\mathbf{M}_{n}\right)$ of 31 $\mathrm{kDa}$ and a dispersity $(Ð)$ of 2.02. Separately, the ethoxy derivative of pillar[5]arene was prepared via condensation of 1,4-diethoxybenzene with paraformaldehyde in the presence of $\mathrm{BF}_{3} \bullet \mathrm{OEt}_{2}$ (Scheme $\mathrm{S} 3$ ). The resulting structure was then mono-de-ethylated using $\mathrm{BBr}_{3}$, 
M.Sc. Thesis - C. Shamshoom - McMaster University- Chemistry and Chemical Biology

resulting in the mono-hydroxylated pillar[5]arene $\mathbf{6}$. This moiety was introduced into the conjugated polymer scaffold by coupling PF-Br with 6 via phase transfer alkylation, to afford the pillar[5]arene-containing polyfluorene, PF-Pillar (Scheme 6). As shown in Figure 11, the ${ }^{1} \mathrm{H}$ NMR signal at $3.30 \mathrm{ppm}$ in PF-Br, which corresponds to the methylene group adjacent to the alkyl bromide, shifts to $3.76 \mathrm{ppm}$ in PF-Pillar. This downfield shift of the resonance upon alkylation is indicative of the quantitative conversion from the alkyl bromide to the alkyl ether product.

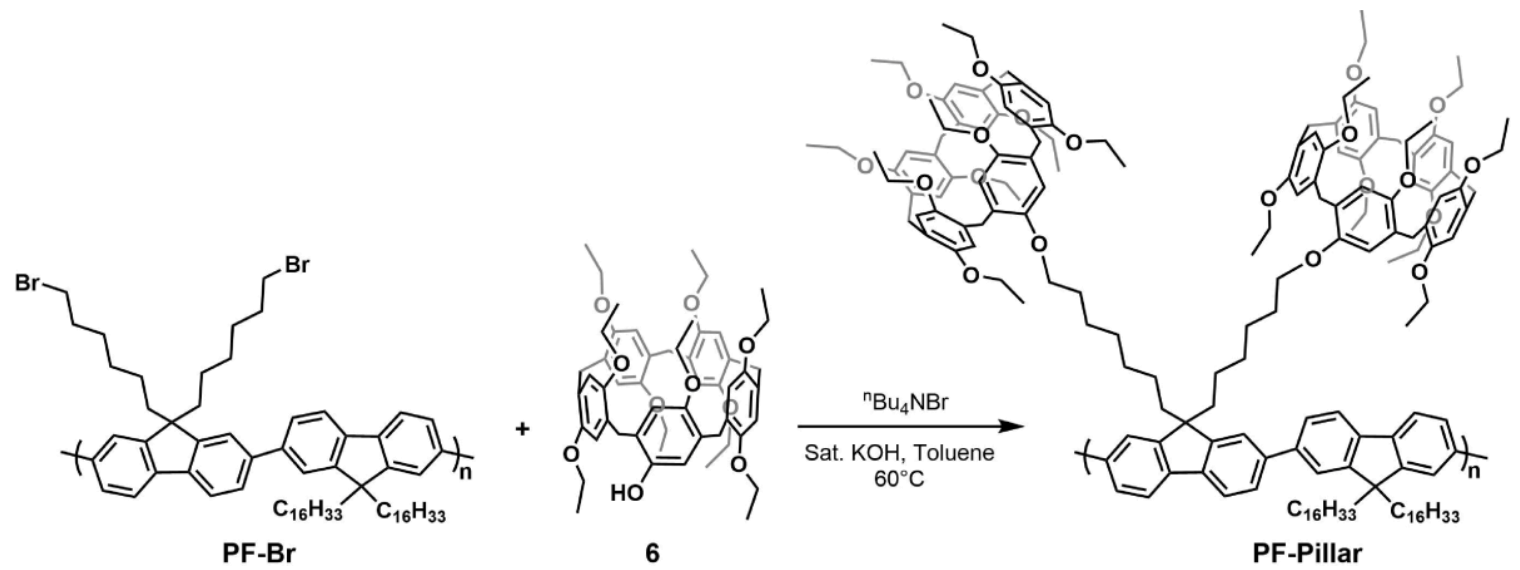

Scheme 6. Post-polymerization functionalization of PF-Br with 6 to afford PF-Pillar.

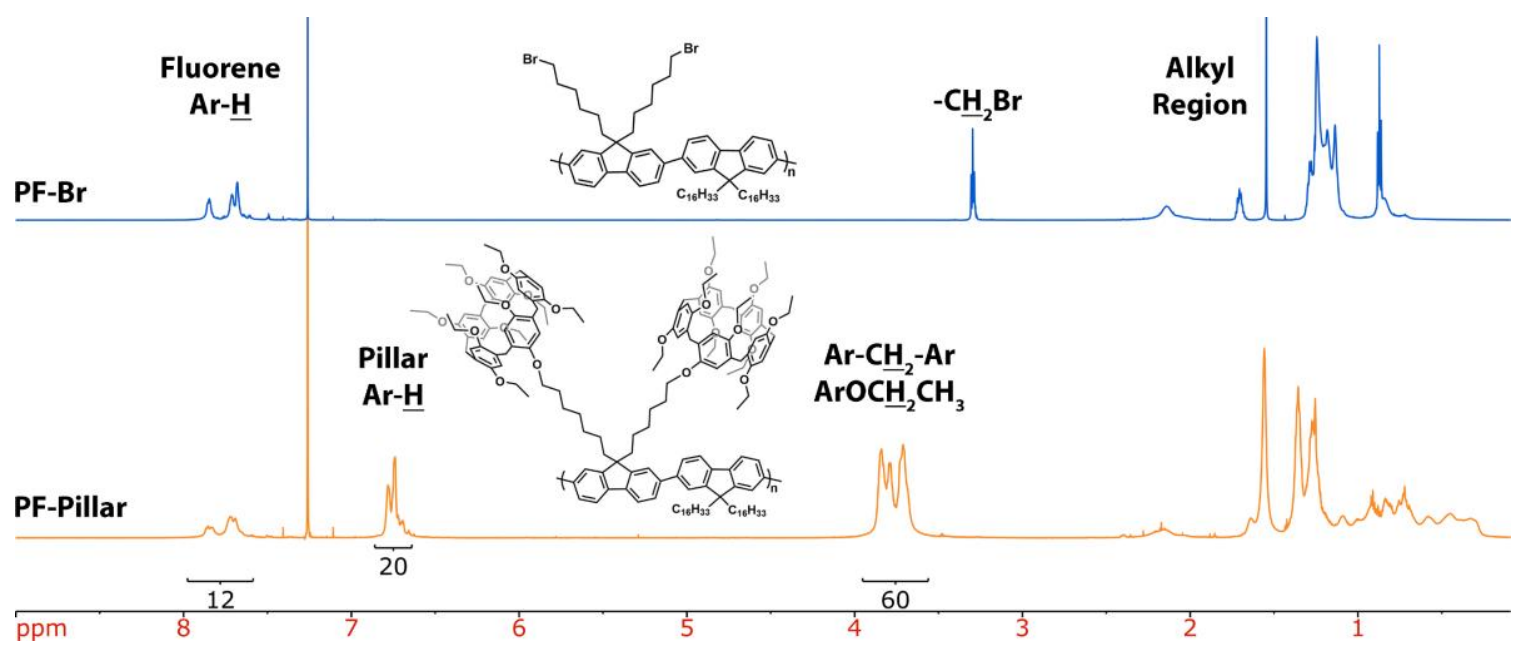

Figure 11. ${ }^{1} \mathrm{H}-\mathrm{NMR}$ spectra overlay of PF-Br (blue) and PF-Pillar (orange) in $\mathrm{CDCl}_{3}$. 
M.Sc. Thesis - C. Shamshoom - McMaster University- Chemistry and Chemical Biology

\subsection{Preparation of Polymer-SWNT Dispersions}

With our polymers in hand, polymer-SWNT dispersions were prepared with raw HiPCO SWNTs following modified literature procedures. ${ }^{159}$ As shown in Figure 12, HiPCO SWNTs (5 mg) were added to a solution of polymer (10 mg of PF-Br or PF-Pillar) in THF $(10 \mathrm{~mL})$. The mixture was then sonicated for $2 \mathrm{~h}$ in a bath sonicator chilled with ice. The resultant black suspension was centrifuged at $8,346 \mathrm{~g}$ for $30 \mathrm{~min}$, and the supernatant was carefully isolated to obtain the polymer-SWNT dispersion, which was stable on the benchtop for at least several months.

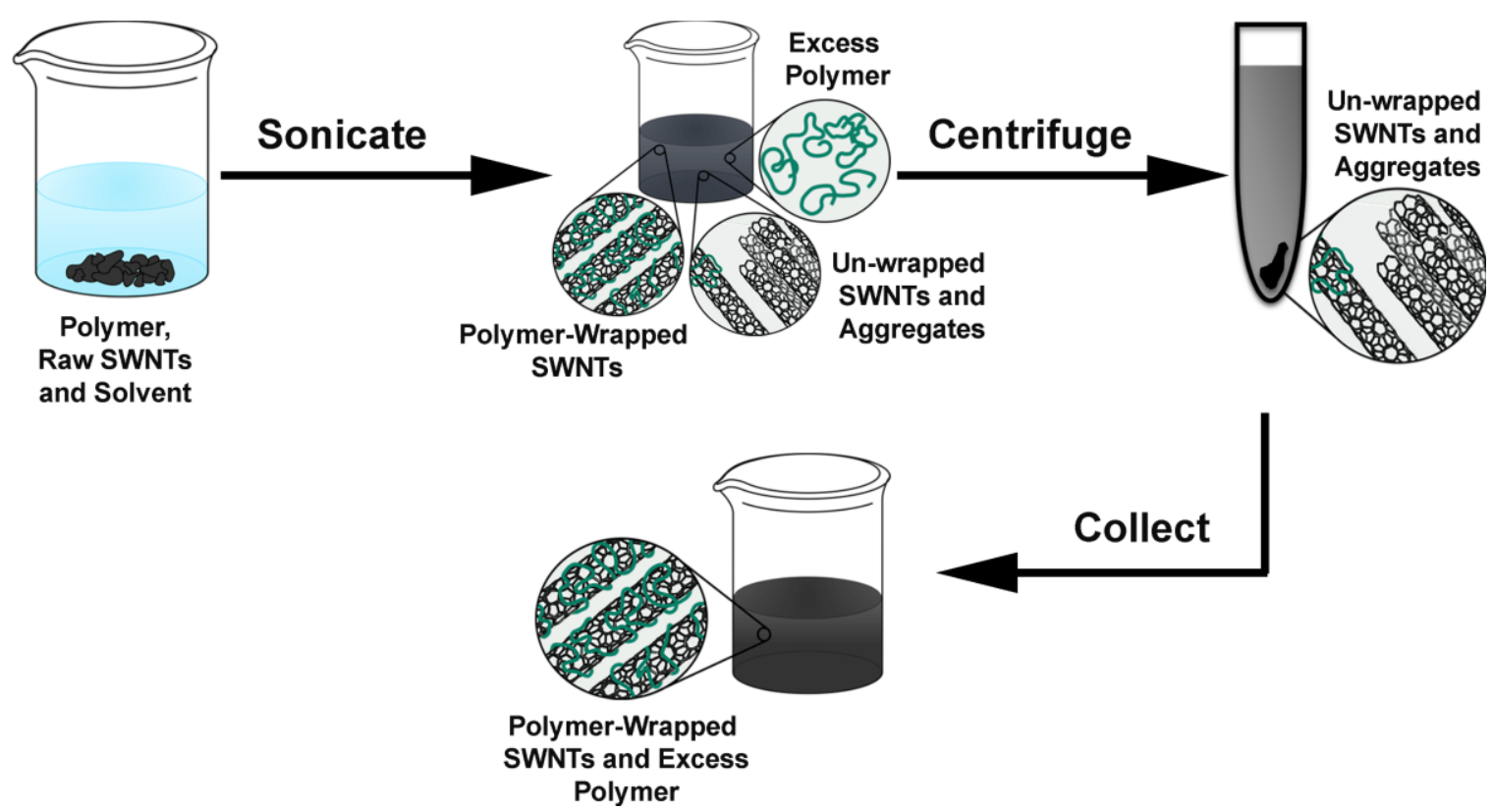

Figure 12. Protocol for preparation of polymer-wrapped-SWNT dispersions in organic solvent.

\subsection{Characterization of the Polymer-SWNT Dispersions}

To characterize the polymer-SWNT dispersion, we first performed UV-Vis-NIR spectroscopy. The absorption features within the spectral range can be grouped into three 
M.Sc. Thesis - C. Shamshoom - McMaster University- Chemistry and Chemical Biology

categories: Two semiconducting regions, $S_{11}(830-1600 \mathrm{~nm})$ and $S_{22}(600-800 \mathrm{~nm})$, and a metallic region, $\mathrm{M}_{11}(440-645 \mathrm{~nm}) .{ }^{71}$ As shown in Figure 2, the as-produced PF-BrSWNT dispersion possesses sharp absorption features that reach a maximum intensity of 0.9. The absence of a broad exponential background suggests at least some degree of scSWNT species enrichment. In contrast, the as-produced PF-Pillar-SWNT dispersion was highly concentrated and exhibited absorptions in the metallic as well as the semiconducting regions (Figure 13). The as-produced PF-Pillar-SWNT dispersion was too concentrated for measurement and required a five-fold dilution to obtain the absorption spectrum shown in Figure 13. We hypothesize that steric bulk imparted by the pillar[5] arene side chains allows for effective steric stabilization of the colloidal polymer-SWNT dispersion.

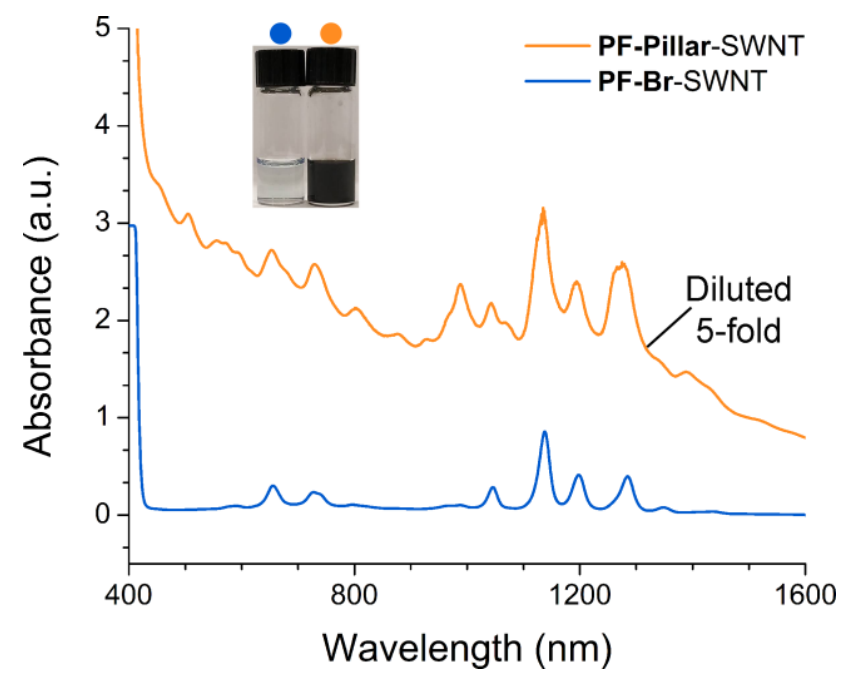

Figure 13. UV-Vis-NIR absorption spectra of polymer-SWNT dispersions (2:1 polymer:SWNT mass ratio) in THF for PF-Pillar-SWNT (orange) and PF-Br-SWNT (blue). The absorption spectrum for PF-Pillar-SWNT was diluted five-fold in THF.

To further probe the polymer-SWNT dispersions, we employed TGA to calculate SWNT concentration. Samples for TGA were prepared by filtering a known aliquot of polymer-SWNT dispersion $(0.5-6 \mathrm{~mL})$ through a Teflon membrane with $0.2 \mu \mathrm{m}$ pore 
M.Sc. Thesis - C. Shamshoom - McMaster University- Chemistry and Chemical Biology

diameter and then washing with THF until the filtrate did not fluoresce at $365 \mathrm{~nm}$. The samples were then transferred to the TGA crucible to determine the recovered mass of polymer-SWNT complex. As shown in Figure S1, samples were heated to $500{ }^{\circ} \mathrm{C}$ under an argon atmosphere at a rate of $15^{\circ} \mathrm{C} \cdot \mathrm{min}^{-1}$. Polymer-only samples were also recorded under identical experimental conditions, and the mass losses, which correspond to polymer side chain degradation, could be used to calculate the SWNT mass fraction $\left(f_{S W N T}\right)$ (see Appendix C for calculations). Given a known volume of polymer-SWNT dispersion $\left(V_{\text {polymer-SWNT }}\right)$ containing a known mass of polymer-SWNT complex $\left(m_{\text {polymer-SWNT }}\right)$, the SWNT concentration $\left(c_{S W N T}\right)$ could be calculated as:

$$
c_{S W N T}=\frac{f_{S W N T} \times m_{\text {polymer }-S W N T}}{V_{\text {polymer }-S W N T}}
$$

The relevant data are tabulated in Table S1, and $c_{S W N T}$ for the PF-Br-SWNT and PF-Pillar-SWNT dispersions were determined to be 20 and $600 \mu \mathrm{g} \cdot \mathrm{mL}^{-1}$, respectively. These results demonstrate that the introduction of macrocyclic structures onto the polymer side chains is effective at producing unusually concentrated SWNT dispersions using small amounts of polymer ( 30-fold increase in SWNT concentration using a 2:1 polymer:SWNT mass ratio). In comparison, a number of reports describe polymer:SWNT mass ratios in excess of 50:1, only to produce relatively dilute dispersions. ${ }^{160,161}$

We then performed Raman spectroscopy to investigate the differences in the SWNT populations dispersed by PF-Br and PF-Pillar. The samples were prepared by dropcasting the dispersions onto a silicon wafer and evaporating the solvent at room temperature. A reference sample containing raw HiPCO SWNTs was prepared by sonicating the raw SWNTs in $\mathrm{CHCl}_{3}$ and then drop-casting the suspension onto a silicon 
M.Sc. Thesis - C. Shamshoom - McMaster University- Chemistry and Chemical Biology

wafer. Raman scans were obtained using excitation wavelengths at 514, 633, and $785 \mathrm{~nm}$, as it has been shown that these excitation wavelengths are sufficient for the characterization of the electronic properties of HiPCO SWNTs. ${ }^{162}$ Figure 14 shows the radial breathing mode (RBM) of the Raman spectra at the three excitation wavelengths (full Raman spectra are provided in Figure S2). The spectra were normalized to the G-band at $\sim 1590 \mathrm{~cm}^{-1}$ and offset for clarity. In the RBM region at $514 \mathrm{~nm}$, predominantly m-SWNT features (225 to $290 \mathrm{~cm}^{-1}$ ) are observed. ${ }^{163}$ The PF-Pillar-SWNT sample exhibits peaks in this region, while the PF-Br-SWNT sample does not (Figure 14a). This suggests that, under identical dispersion preparation conditions, PF-Pillar disperses m-SWNTs, while PF-Br does not. This result is further corroborated by G-band analysis (inset of Figure 14a). The G-band consists of two peaks: A lower frequency $\mathrm{G}^{-}$and a higher frequency $\mathrm{G}^{+}$. For sc-SWNTs, both the $\mathrm{G}^{-}$and $\mathrm{G}^{+}$have Lorentzian line shapes, while for $\mathrm{m}-\mathrm{SWNT}$ the $\mathrm{G}^{-}$exhibits a broader Breit-Wigner-Fano (BWF) line shape. ${ }^{86} \mathrm{~A}_{\text {broad }} \mathrm{G}^{-}$band is observed for the PFPillar-SWNT sample, which is consistent with the presence of m-SWNTs. Meanwhile, the PF-Br-SWNT sample lacks the BWF line shape in the G-band, confirming that m-SWNTs are absent. At $633 \mathrm{~nm}$, both m-SWNTs $\left(175-230 \mathrm{~cm}^{-1}\right)$ and sc-SWNTs $\left(230-300 \mathrm{~cm}^{-1}\right)$ are in resonance. ${ }^{86,164}$ As shown in Figure 14b, the PF-Pillar-SWNT sample exhibits peaks corresponding to both sc- and m-SWNTs. However, only sc-SWNTs peaks are present in the PF-Br-SWNT sample. Lastly, at $785 \mathrm{~nm}$ sc-SWNTs are primarily in resonance. ${ }^{163,165}$ A few large diameter metallic species, most notably the $(16,7)$ and $(12,9)$ species, are also observed in the low-frequency region. Figure 14c shows that sc-SWNTs are present in both the PF-Br-SWNT and PF-Pillar-SWNT samples. Again, this demonstrates that PF-Br 
M.Sc. Thesis - C. Shamshoom - McMaster University- Chemistry and Chemical Biology

disperses only sc-SWNTs, while PF-Pillar disperses both sc- and m-SWNTs. Beyond scSWNT peaks at $785 \mathrm{~nm}$, a prominent peak at $265 \mathrm{~cm}^{-1}$ arises from the $(10,2) \mathrm{sc}-\mathrm{SWNT}$ species when trapped in a SWNT bundle. Assuming $(10,2)$ is present, this "bundling peak" can be used to identify the degree of bundling in a nanotube sample. ${ }^{159}$ It was found that the bundling peak for both polymer-SWNT samples was substantially supressed compared to the raw SWNT sample. Overall, the Raman data demonstrates that the concentrated PFPillar-SWNT sample is a function of unusual colloidal stability, rather than due to the suspension of SWNT bundles.
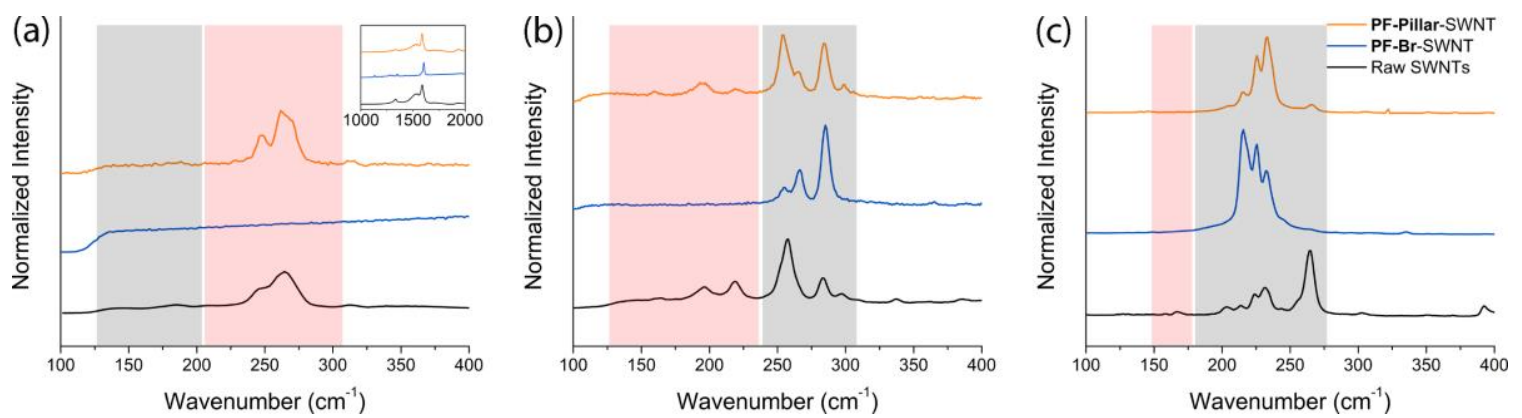

Figure 14. RBM regions at (a) 514, (b) 633, and (c) $785 \mathrm{~nm}$ excitation wavelengths. Raman spectra The gray boxes denote the signals arising from sc-SWNTs, while the pink boxes indicate the locations of signals from m-SWNTs. The inset in (a) shows the G-band region, located at $\sim 1590 \mathrm{~cm}^{-1}$, upon excitation at $514 \mathrm{~nm}$.

To further characterize the polymer-SWNT dispersions, photoluminescence (PL) maps were obtained. The polymer-SWNT dispersions were diluted in THF to obtain an absorption intensity of $\sim 0.11$ for the peak centred at $\sim 1279 \mathrm{~nm}$ (Figure S3). The excitation and emission energies of various sc-SWNTs were obtained from experimental Kataura plots and plotted on the PL map. ${ }^{74}$ As shown in Figure 15a, high intensity PL signals were observed in the PF-Br-SWNT dispersion, with the most intense peak corresponding to the $(7,6)$ sc-SWNT species. Other prominent species include $(8,7),(8,6)$, and $(7,5)$. For the PF- 
M.Sc. Thesis - C. Shamshoom - McMaster University- Chemistry and Chemical Biology

Pillar-SWNT dispersion, the $(7,6)$ species is also the most intense species. Other prominent species include $(9,4),(7,5),(6,5)$, and $(8,4)$. Interestingly, the relative fluorescence intensity of the PF-Pillar-SWNT sample is roughly two orders of magnitude lower compared to the PF-Br-SWNT sample. The reduced fluorescence intensity in PF-PillarSWNT may be attributed to either SWNT bundles or m-SWNT species present in the sample, as both are known fluorescence quenchers. ${ }^{11}$ As Raman analysis indicates the lack of significant bundling in the polymer-SWNT dispersions, we attribute the observed fluorescence quenching to an increased amount of m-SWNTs dispersed by PF-Pillar, which is consistent with the aforementioned analyses.
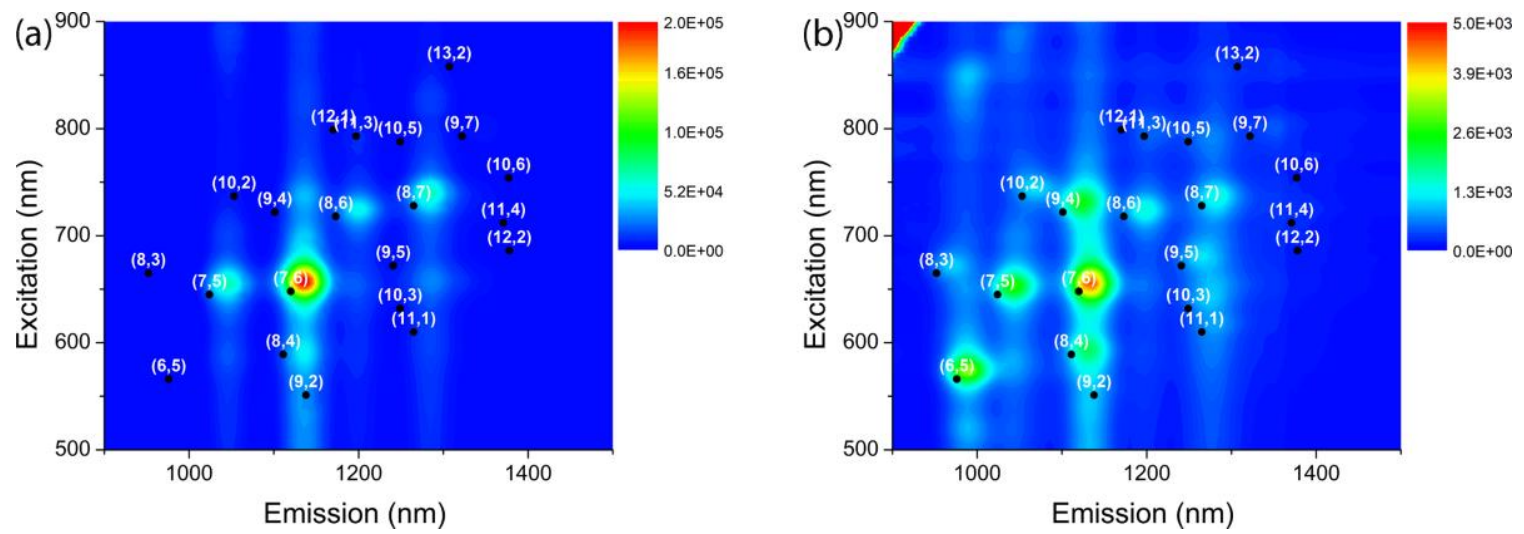

Figure 15. Photoluminescence maps of (a) PF-Br-SWNT and (b) PF-Pillar-SWNT, concentration-matched by UV-Vis-NIR and plotted on the same scale.

\subsection{Host-Guest Properties of Polymer-SWNT Dispersion}

With our polymer-SWNT dispersions fully characterized, we sought to explore the dispersion properties of the PF-Pillar-SWNT sample using ${ }^{1} \mathrm{H}-\mathrm{NMR}$ spectroscopy. We employed ${ }^{1} \mathrm{H}-\mathrm{NMR}$ to investigate the host-guest interactions in the presence of our polymer-SWNT sample. We first examined the host-guest interactions between PF-Pillar and 1,6-dicyanohexane in THF-d $\mathrm{d}_{8}$ (Figure S4). The resonance at $6.84 \mathrm{ppm}$, which 
M.Sc. Thesis - C. Shamshoom - McMaster University- Chemistry and Chemical Biology

corresponds to the aromatic protons in pillar[5]arene, was shifted downfield by $0.07 \mathrm{ppm}$ upon the addition of 1,6-dicyanohexane. This downfield shift is consistent with the association between 1,6-dicyanohexane and a pillar[5]arene-functionalized conjugated polymer. ${ }^{166}$ To investigate the properties of the PF-Pillar-SWNT complex, we prepared a PF-Pillar-SWNT dispersion in THF-d $8(0.75 \mathrm{~mL})$, following the previously outlined protocol (vide supra), and added hexamethyldisilane $(0.5 \mu \mathrm{L})$ as an internal standard. NMR spectra were normalized to this internal standard (for full ${ }^{1} \mathrm{H}-\mathrm{NMR}$ spectra, see Figure S5). Compared to PF-Pillar, the ${ }^{1} \mathrm{H}-\mathrm{NMR}$ spectrum of the PF-Pillar-SWNT dispersion in THF- $\mathrm{d}_{8}$ shows broad signals which correspond to the fluorene backbone as well as the pillar[5]arene moiety (Figure 16). Upon addition of 1,6-dicyanohexane, the resonance corresponding to the aromatic pillar[5] arene protons shifts from $6.83 \mathrm{ppm}$ to $6.91 \mathrm{ppm}$, with slight broadening. Thus, it is apparent that the host-guest chemistry between the pillar[5]arene moiety and 1,6-dicyanohexane occurs in the presence of the polymer-SWNT complex. 
M.Sc. Thesis - C. Shamshoom - McMaster University- Chemistry and Chemical Biology

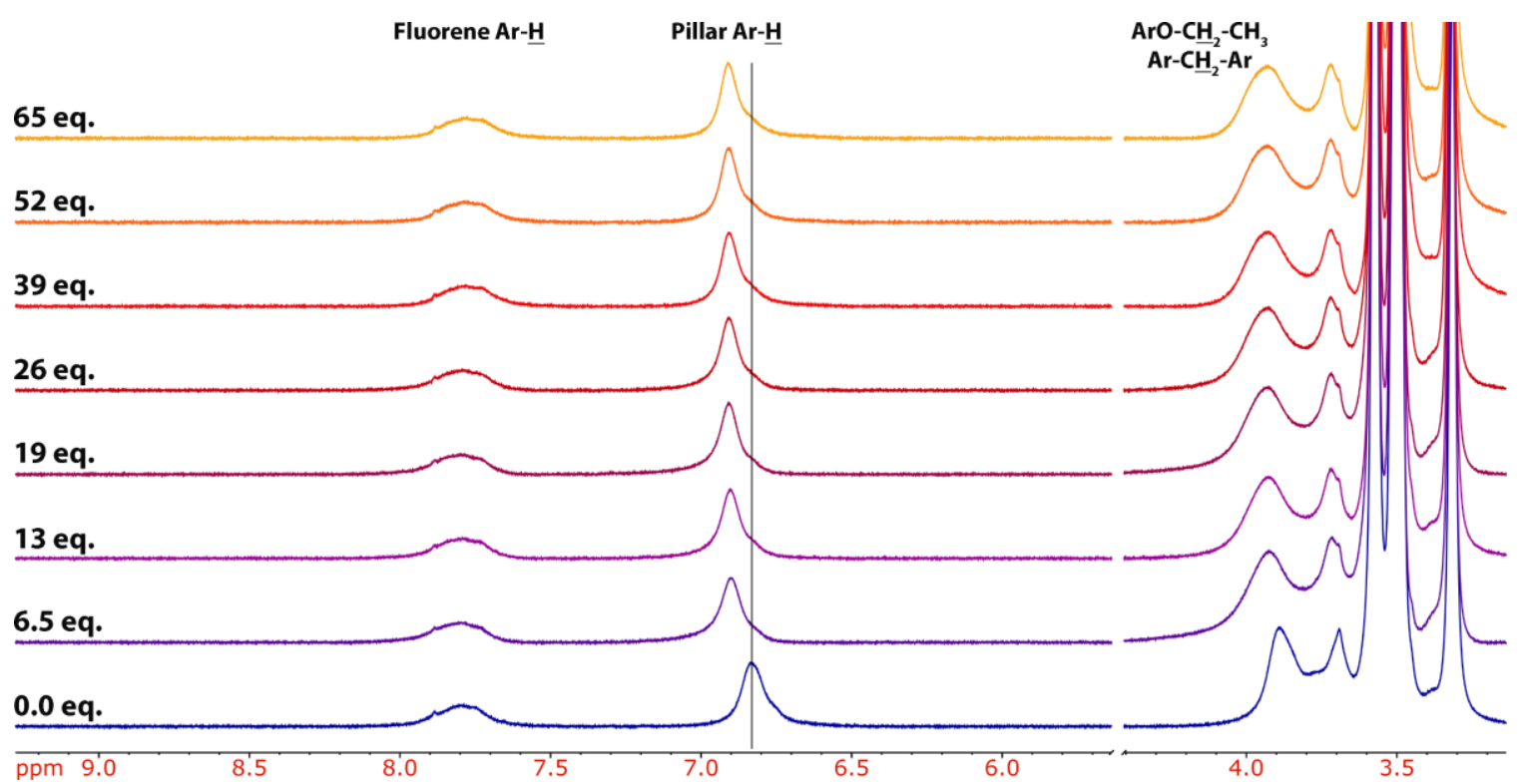

Figure 16. ${ }^{1} \mathrm{H}-\mathrm{NMR}$ spectra $\left(\mathrm{THF}-\mathrm{d}_{8}, 298 \mathrm{~K}\right)$ of the PF-Pillar-SWNT dispersion recorded after successive additions of 1,6-dicyanohexane $(0-65$ eq).

\subsection{Summary}

This chapter explored the synthesis and characterization of a macrocyclecontaining conjugated polymer as well as its effect on the solubility of carbon nanotubes. The post-polymerization functionalization of polyfluorene bromide was utilized to prepare a pillar[5]arene-derivatized polyfluorene, as evidenced by ${ }^{1} \mathrm{H}-\mathrm{NMR}$ spectroscopy. UVVis-NIR spectroscopy and thermogravimetric analysis showed that the non-covalent functionalization of SWNTs with the macrocycle containing polyfluorene significantly enhanced nanotube solubility. This resulted in a dark and concentrated nanotube dispersion, compared to that of the polyfluorene bromide precursor. Also, analysis of the polymer-SWNT dispersions by UV-Vis-NIR, Raman, and Photoluminescence spectroscopy revealed that the initial polyfluorene derivative preferentially dispersed scSWNT species, while the pillar[5]arene-decorated polyfluorene produced dispersions 
M.Sc. Thesis - C. Shamshoom - McMaster University- Chemistry and Chemical Biology

containing both sc-SWNTs and m-SWNTs. The host-guest properties of the pillar[5]arenedecorated polyfluorene SWNT dispersion was investigated using ${ }^{1} \mathrm{H}-\mathrm{NMR}$ spectroscopy. It was found that the formation of an inclusion complex between pillar[5]arene and 1,6dicyanohexane occurred in the presence of the macrocycle-containing polymer-SWNT complex. 
M.Sc. Thesis - C. Shamshoom - McMaster University- Chemistry and Chemical Biology

\section{Chapter 3: Preparation and Characterization of Supramolecular Organogels}

The experiments outlined in Chapter 3 build on the host-guest properties of the macrocycle-containing polymer-SWNT complex. Host-guest interactions formed with pillar[5]arenes have found applications in materials such as polymers, ${ }^{112-114}$ sensors, ${ }^{108,115,116}$ and organogels. ${ }^{104,117,118}$ Recently, we have explored the host-guest interactions of a pillar[5]arene functionalized conjugated polyimine scaffold with various alkylnitrile guests. ${ }^{166} \mathrm{We}$ prepared self-healing organogels made by the pillar[5]arenealkylnitrile inclusion complexes. However, the gels were mechanically weak due to the inherently weaker crosslinks between the pillar[5]arene and alkylnitrile polymer chains. ${ }^{167,168}$ Organogel stiffness can be improved via the introduction of a reinforcer such as carbon nanotubes. ${ }^{169-171}$ Considering that carbon nanotubes have a high Young's Modulus (up to $1 \mathrm{TPa}$ ) and a high aspect ratio, they may be effective for reinforcement with minimal SWNT loading. ${ }^{172-175}$ This chapter explores the incorporation of the pillar[5]arene-containing polymer-SWNT complex into a supramolecular organogel. Chapter 3 focuses on the preparation and characterization of the supramolecular organogels, beginning with the synthesis of a crosslinking polymer containing terminal alkyl nitriles. Then, we explore its interaction with the pillar[5]arene-decorated polyfluorene to generate a supramolecular organogel. Finally, we investigate the difference in mechanical properties of the organogel before and after SWNT incorporation. 
M.Sc. Thesis - C. Shamshoom - McMaster University- Chemistry and Chemical Biology

\subsection{Preparation of Supramolecular Organogels}

Having confirmed the formation of inclusion complexes in the presence of the PFPillar-SWNT dispersion (Chapter 2.5), we sought to incorporate the concentrated dispersion into supramolecular organogels. Initially, we envisioned a crosslinked network of pillar moieties on PF-Pillar with a polyfluorene containing alkyl nitrile side chains (PFCN) through combination of equimolar ratios of PF-Pillar and PF-CN. We expected the pillar[5]arene structures on the PF-Pillar to form inclusion complexes with the alkyl nitriles of PF-CN, making a "zipper-like” organogel (Figure 17).
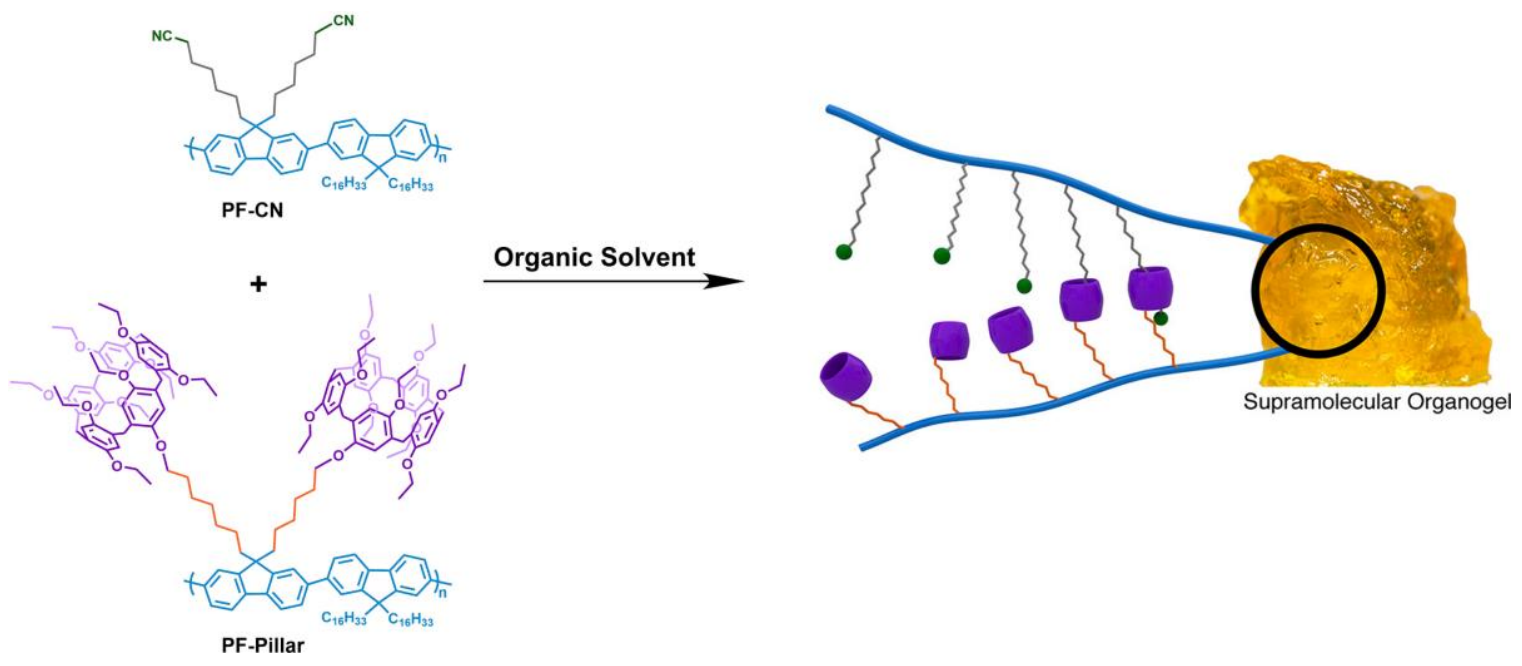

Figure 17. Cartoon representation of host-guest driven gelation of PF-Pillar (purple) and PF-CN (green). Adapted from [Kardelis, V.; Li, K.; Nierengarten, I.; Holler, M.; Nierengarten, J. F.; Adronov, A. Macromolecules 2017, 50 (23), 9144-9150]. ${ }^{166}$

Thus, we attempted to synthesize a functionalized polymer via cyanide substitution of the alkyl bromide on PF-Br. Initially, we reacted PF-Br (in THF) in the presence of potassium cyanide $(\mathrm{KCN})$ in dimethyl formamide (Scheme 7). The reaction was unsuccessful as there was no observed shift of the methylene group protons adjacent to the bromide in PF-Br by ${ }^{1} \mathrm{H}-\mathrm{NMR}$ spectroscopy. We hypothesized that the cyanide ion was 
M.Sc. Thesis - C. Shamshoom - McMaster University- Chemistry and Chemical Biology

poorly soluble in the organic phase and as a result the substitution reaction did not occur. Alternatively, we dissolved the potassium cyanide in water, added tetrabutyl ammonium bromide $\left({ }^{\mathrm{n}} \mathrm{Bu} 4 \mathrm{NBr}\right)$ and attempted a phase-transfer cyanide substitution with $\mathbf{P F}-\mathbf{B r}$ in various organic solvents (Scheme 7). Again, no reaction was observed by ${ }^{1} \mathrm{H}-\mathrm{NMR}$ spectroscopy. It is possible that the tetrabutyl ammonium bromide ion pair is more lipophilic than tetrabutyl ammonium cyanide, thus the cyanide nucleophile does not readily substitute the bromide in the organic phase. ${ }^{176}$ Instead, we performed the alkylation employing tetrabutylammonium hydroxide $\mathrm{TBAOH}$, which is exceedingly less lipophilic than the tetrabutylammonium cyanide ion, ${ }^{176}$ allowing for facile substitution of the bromide in the organic phase. Reaction of PF-Br under these conditions yielded an insoluble product that could not be characterized by ${ }^{1} \mathrm{H}-\mathrm{NMR}$ spectroscopy. 
M.Sc. Thesis - C. Shamshoom - McMaster University- Chemistry and Chemical Biology

(a)

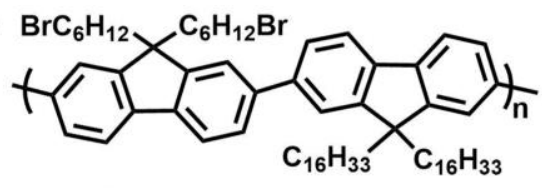

PF-Br

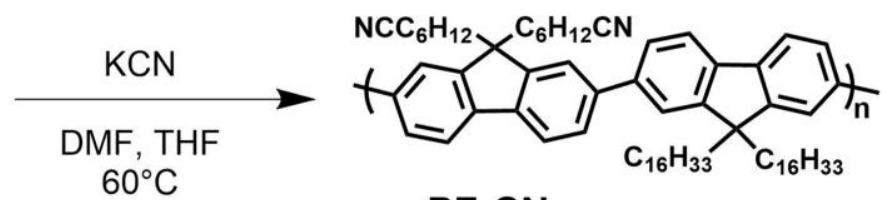

PF-CN

(b)

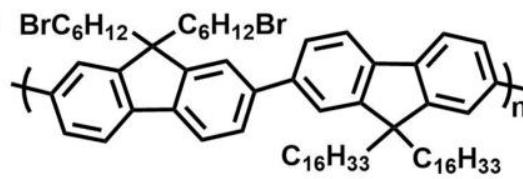

$\mathrm{PF}-\mathrm{Br}$
$\underset{\mathrm{DCM}, \mathrm{H}_{2} \mathrm{O}}{\stackrel{\mathrm{KCN},{ }^{\mathrm{n}} \mathrm{Bu}_{4} \mathrm{NBr}}{\longrightarrow}}$

$60^{\circ} \mathrm{C}$

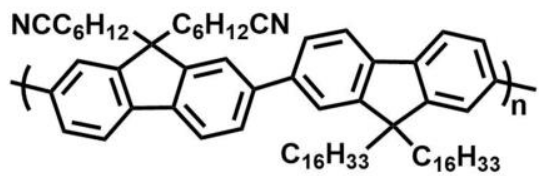

PF-CN

(c)

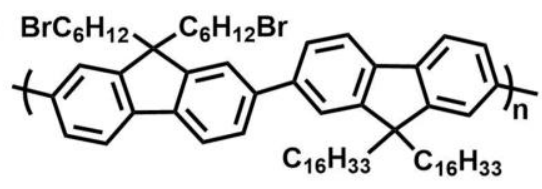

$\mathrm{PF}-\mathrm{Br}$

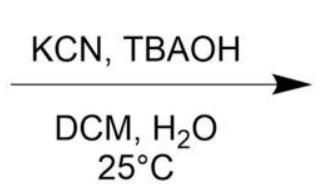

$25^{\circ} \mathrm{C}$

Scheme 7. Various attempts to synthesize polyfluorene containing alkyl nitrile side chains (PF-CN) via cyanide substitution of the alkyl bromide on polyfluorene bromide (PF-Br).

We were unsuccessful in our attempts to make PF-CN, and instead chose a homobifunctional PEG polymer with terminal alkyl nitriles $\left(\mathrm{PEG}_{600}-(\mathrm{CN})_{2}\right)$ to act as a crosslinker. We recently reported the synthesis of $\mathrm{PEG}_{600}-(\mathrm{CN})_{2}$ in good yield from commercially available PEG $_{600}$ and dibromohexane, which formed relatively soft organogels. ${ }^{166}$

Thus, to prepare the organogels, we first synthesized $\mathrm{PEG}_{600}-(\mathrm{CN})_{2}$ to act as a crosslinker, according to literature procedures. ${ }^{166}$ Figure 18 depicts an idealized cartoon representation of the supramolecular gel. We envisioned a system where the pillar[5]arene units interact with the alkyl nitrile groups in the $\mathrm{PEG}_{600}-(\mathrm{CN})_{2}$ crosslinker to form a uniform crosslinked network. 


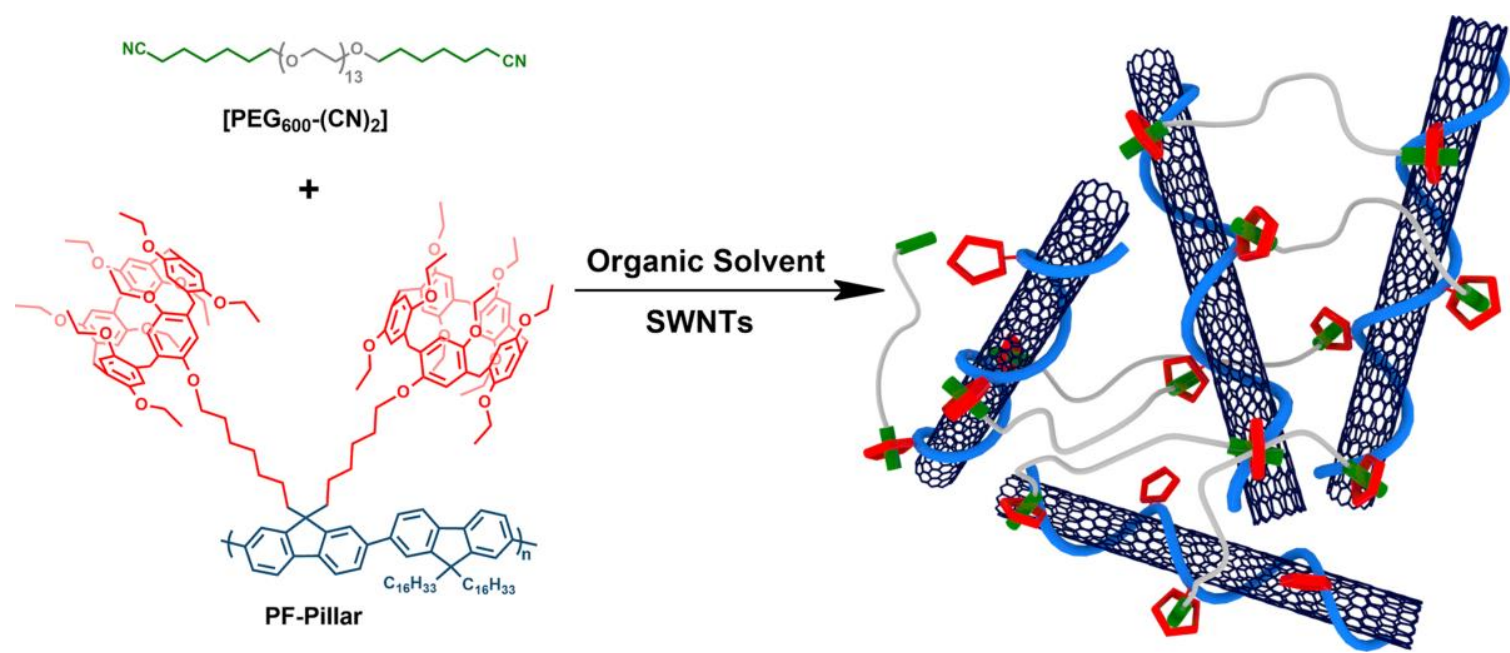

Figure 18. Cartoon representation of host-guest driven gelation of PF-Pillar-SWNT (red) and $\mathrm{PEG}_{600}-(\mathrm{CN})_{2}$ (green).

\subsection{Preparation of Organogels Utilising Solvent Exchange Method}

We attempted to characterize the mechanical properties of the supramolecular organogels. With the organogel successfully made, we sought to explore the effect of added SWNTs on the stiffness of the gel. The Young's Modulus was determined by performing mechanical strength measurements on native (control) and hybrid organogels. Our initial mechanical testing of gels swollen with THF proved to be problematic due to the rapid evaporation of the organic solvent. This made the gels stiffer as the measurement progressed and resulted in inconsistent data.

To mitigate the problem of THF evaporation, we explored alternative low-boiling solvents when preforming mechanical strength testing of the gels. Attempts to make polymer-SWNT complexes in solvents such as toluene, xylene, chlorobenzene and 1,2dichlorobenzene were unsuccessful, as the resultant dispersions were either too dilute or unstable (i.e. SWNT bundles formed immediately after centrifugation). A solvent exchange 
M.Sc. Thesis - C. Shamshoom - McMaster University- Chemistry and Chemical Biology

method was employed to achieve a stable dispersion in a high boiling solvent such as 1,2dichlorobenzene. Briefly, $100 \mu \mathrm{L}$ of PF-Pillar-SWNT suspension in THF was added to $100 \mu \mathrm{L}$ of 1,2 -dichlorobenzene in a $20-\mathrm{mL}$ vial. The mixture was placed under vacuum to evaporate the THF, resulting in a black PF-Pillar-SWNT dispersion in 1,2dichlorobenzene.

To investigate any potential increase in bundling upon solvent exchange, Raman spectroscopy was preformed on thin film samples of SWNT dispersions in THF and 1,2dichlorobenzene. Samples were prepared by drop-casting the dispersions onto silicon wafers. A laser excitation wavelength of $785 \mathrm{~nm}$ was used to characterize the samples. A reference sample containing only raw SWNTs was prepared via sonication in chloroform and drop-casting onto a silicon wafer. Figure 19 shows the radial breathing mode (RBM) region of the Raman spectra for PF-Pillar-SWNT dispersions cast from THF and 1,2dichlorobenzene (full Raman spectra are provided in Figure S6). The spectra were normalized to the G-band at $\sim 1590 \mathrm{~cm}^{-1}$ and offset for clarity. For HiPCO SWNTs at 785 $\mathrm{nm}$, a prominent peak at $265 \mathrm{~cm}^{-1}$ arises from the $(10,2) \mathrm{sc}-\mathrm{SWNT}$ chirality when bundled. This "bundling peak" may be used to identify the degree of bundling in a nanotube sample, assuming $(10,2)$ is present. ${ }^{177}$ The samples show a significant suppression of the bundling peak when HiPCO SWNTs were dispersed with PF-Pillar in THF. The degree of bundling was quantified by integrating the bundling peak $\left(252-280 \mathrm{~cm}^{-1}\right)$ relative to the SWNT peaks $\left(208-248 \mathrm{~cm}^{-1}\right)$ and comparing the relative intensities (integrations are tabulated in Table 1). Upon solvent exchange with 1,2-dichlorobenzene (by removing THF), the bundling peak intensity remained identical to the PF-Pillar-SWNT in THF (Table 1). To 
M.Sc. Thesis - C. Shamshoom - McMaster University- Chemistry and Chemical Biology

corroborate the results of the Raman experiment, we recorded a PL map to confirm the presence of $(10,2)$ sc-SWNTs. The PL map of PF-Pillar-SWNT (Figure 15, Section 2.4) shows a moderate intensity PL signal corresponding to $(10,2)$ sc-SWNTs in the polymerSWNT sample. As $(10,2)$ is present, we can confirm that the Raman bundling analysis above is valid. ${ }^{74}$

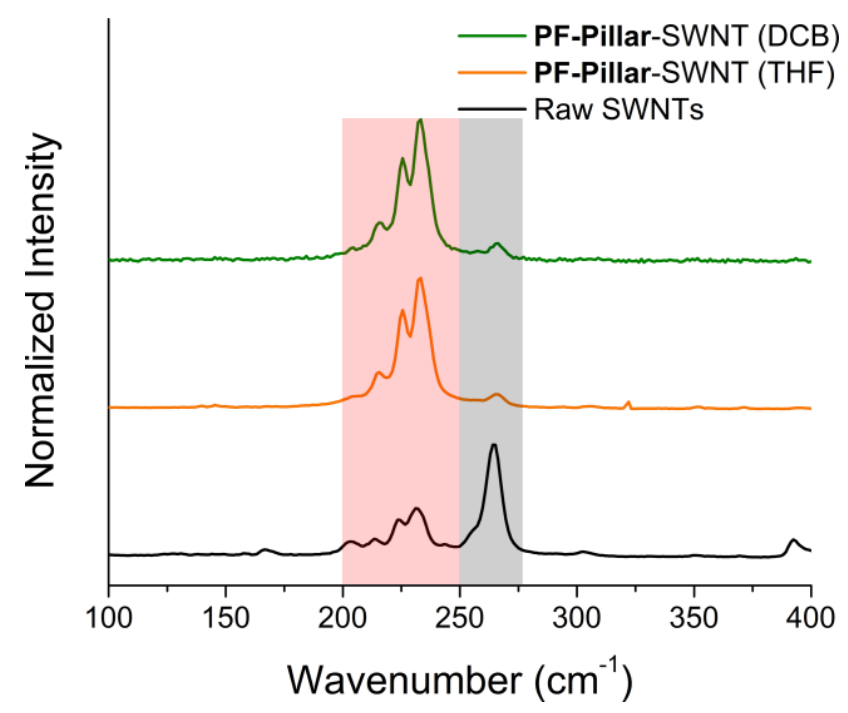

Figure 19. RBM regions of the Raman spectra of PF-Pillar-SWNT samples in THF and upon solvent exchange into 1,2-dichlorobenzene (DCB), compared with raw HiPCO SWNTs. The gray and pink boxes denote the regions of integrated bundling peaks and other SWNT chirlaities, respectively. The spectra were collected at $785 \mathrm{~nm}$ excitation wavelength, normalized to the G-band at $\sim 1590 \mathrm{~cm}^{-1}$ and offset for clarity.

Table 1. Raman integration values of SWNT (208-248 $\left.\mathrm{cm}^{-1}\right)$ and Bundling Peak (BP 252$280 \mathrm{~cm}^{-1}$ ) of various SWNT dispersions and their relative ratios.

\begin{tabular}{cccc} 
Name & $\begin{array}{c}\text { SWNT } \\
\left(\mathbf{2 0 8 - 2 4 8} \mathbf{~ c m}^{-1}\right)\end{array}$ & $\begin{array}{c}\text { BP } \\
\left(\mathbf{2 5 2 - 2 8 0} \mathbf{~ c m}^{-\mathbf{1}}\right)\end{array}$ & $\begin{array}{c}\text { SWNT: BP Ratio } \\
\text { (Relative) }^{\mathbf{1}}\end{array}$ \\
\hline PF-Pillar-SWNT (DCB) & 7.9 & 0.3 & $100: 4$ \\
PF-Pillar-SWNT (THF) & 7.5 & 0.3 & $100: 4$ \\
Raw SWNTs & 2.3 & 2.7 & $100: 116$ \\
\hline
\end{tabular}

1: Ratios were obtained through division of BP integrations by SWNT peak integration values and multiplication by 100 . 
M.Sc. Thesis - C. Shamshoom - McMaster University- Chemistry and Chemical Biology

Using this concept, we prepared 40 wt. \% organogels in 1,2-dichlorobenzene by adding PEG $_{600}-(\mathrm{CN})_{2}$ crosslinker to the PF-Pillar-SWNT dispersion. The organogel samples were prepared inside a $2 \mathrm{~mL}$ microcentrifuge tube and incubated under ambient conditions for 30 min before inversion. The native organogel was prepared by combining PF-Pillar $(22 \mathrm{mg}, 8.2 \mu \mathrm{mol})$ with PEG$_{600}-(\mathrm{CN})_{2}(7 \mathrm{mg}, 8.2 \mu \mathrm{mol})$ in 1,2-dichlorobenzene $(50 \mu \mathrm{L})$. The hybrid, SWNT-containing organogel was prepared by first taking an aliquot of the pristine PF-Pillar-SWNT dispersion, adding the same volume of 1,2dichlorobenzene, and then removing the THF in vacuo. The control organogel was prepared by combining PF-Pillar $(22 \mathrm{mg}, 8.2 \mu \mathrm{mol})$ to $\mathrm{PEG}_{600}(7 \mathrm{mg}, 8.2 \mu \mathrm{mol})$ in $1,2-$ dichlorobenzene $(50 \mu \mathrm{L})$.

After 30 minutes of incubation under ambient conditions, both the PF-PillarPEG $_{600}-(\mathrm{CN})_{2}$ (native) and PF-Pillar-SWNT-PEG $600-(\mathrm{CN})_{2}$ (hybrid) mixtures formed gels that did not flow when the microcentrifuge tube was inverted, as shown in Figure 20. The resultant gels were soft and tacky in texture, with a marked difference in colour between the native (yellow) and hybrid (black) gels. In a control experiment, PF-Pillar and PEG 600 were mixed in 1,2-dichlorobenzene under conditions identical to those used to form the aforementioned organogels. Upon inversion, this control mixture remained liquid and flowed down the wall of the container (Figure 20). 
M.Sc. Thesis - C. Shamshoom - McMaster University- Chemistry and Chemical Biology

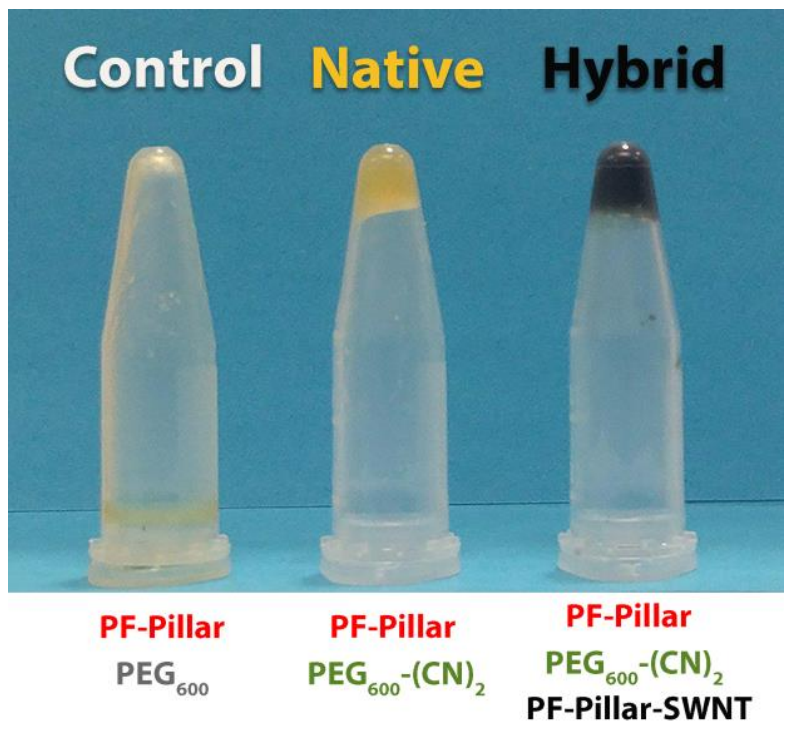

Figure 20. Photographs of supramolecular organogels made from the host-guest interaction between PF-Pillar and PEG600- $(\mathrm{CN})_{2}$ (native) and in the presence of SWNTs (hybrid).

\subsection{Mechanical Testing of Organogels Utilising the CellScale Microsquisher}

Having confirmed the formation of a supramolecular organogel in the presence of the PF-Pillar-SWNT dispersion, we sought to investigate the mechanical properties of the native and hybrid gels. The mechanical testing of the native and hybrid organogels was initially attempted with a CellScale Microsquisher at $22^{\circ} \mathrm{C}$. This instrument is designed to apply a compressive load to specimens 50 to $2000 \mu \mathrm{m}$ in size. ${ }^{178}$ Organogel samples were prepared inside a modified $1 \mathrm{~mL}$ polypropylene syringe and allowed to incubate for 20 minutes before extruding into a round Teflon mold (4 mm diameter and $3 \mathrm{~mm}$ deep) for mechanical testing.

Figure 21 shows a photograph of the CellScale Microsquisher with the following major components: the microbeam, actuators, imaging system, and the fluid bath. The 
M.Sc. Thesis - C. Shamshoom - McMaster University- Chemistry and Chemical Biology

microbeam is a circular tungsten beam with a Modulus of $411000 \mathrm{MPa}$ and is available in a variety of diameters, ranging from 0.0762 to $0.5588 \mu \mathrm{m} .{ }^{178}$ At the end of the microbeam shaft is a rigidly attached compression plate (or a stainless-steel ball may also be attached). The microbeam is rigidly fixed to the actuator, which aids in positioning the microbeam above a specimen. Then, the microbeam applies a deformation to the specimen while measuring the applied force. The applied force is determined by calculating the deflection of the microbeam (difference between the displacement of the tip and the displacement of the actuator). The deformation of the sample is monitored with an imaging system that is composed of a digital camera. The fluid bath is utilized for specialized temperature testing of samples. ${ }^{178}$ This feature was not utilized in the testing of the supramolecular organogels considering that we sought to determine the mechanical properties at room temperature.

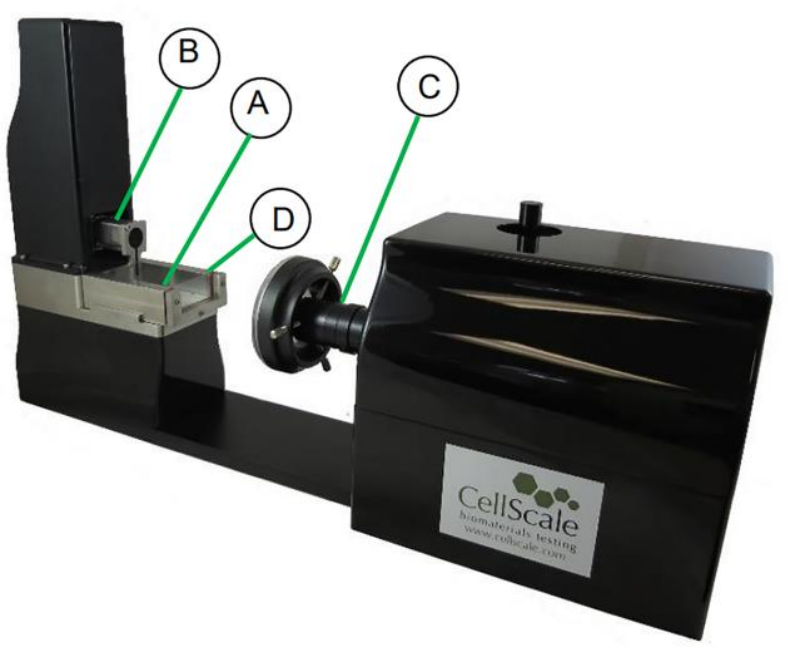
A- Microbeam
B-Actuators
C- Imaging System D- Fluid Bath

Figure 21. Photograph of the CellScale Microsquisher. Adapted from [CellScale. MicroSquisher Micro-scale Tension-Compression Test System]. ${ }^{178}$ 
M.Sc. Thesis - C. Shamshoom - McMaster University- Chemistry and Chemical Biology

Figure 22 illustrates a typical test sequence achieved upon the application and release of a load on the specimen using the Microsquisher. The test sequence may be composed of numerous sets of test cycles. Each test cycle may be divided into the load, hold, recover, and rest phase. ${ }^{178}$ The load phase encompasses the applied deformation to the specimen. The deformation may be expressed either in terms of the force applied or the displacement achieved. Upon completion of the load phase, the deformation is held on the specimen for a desired period of time. The recovery phase begins upon removal of the applied force onto the specimen. The recovery phase is followed by the rest phase, which is the time between the end of one cycle and the beginning of the next. The duration of the various test phases is configurable and dependent on the nature of the testing. ${ }^{178}$ 
M.Sc. Thesis - C. Shamshoom - McMaster University- Chemistry and Chemical Biology

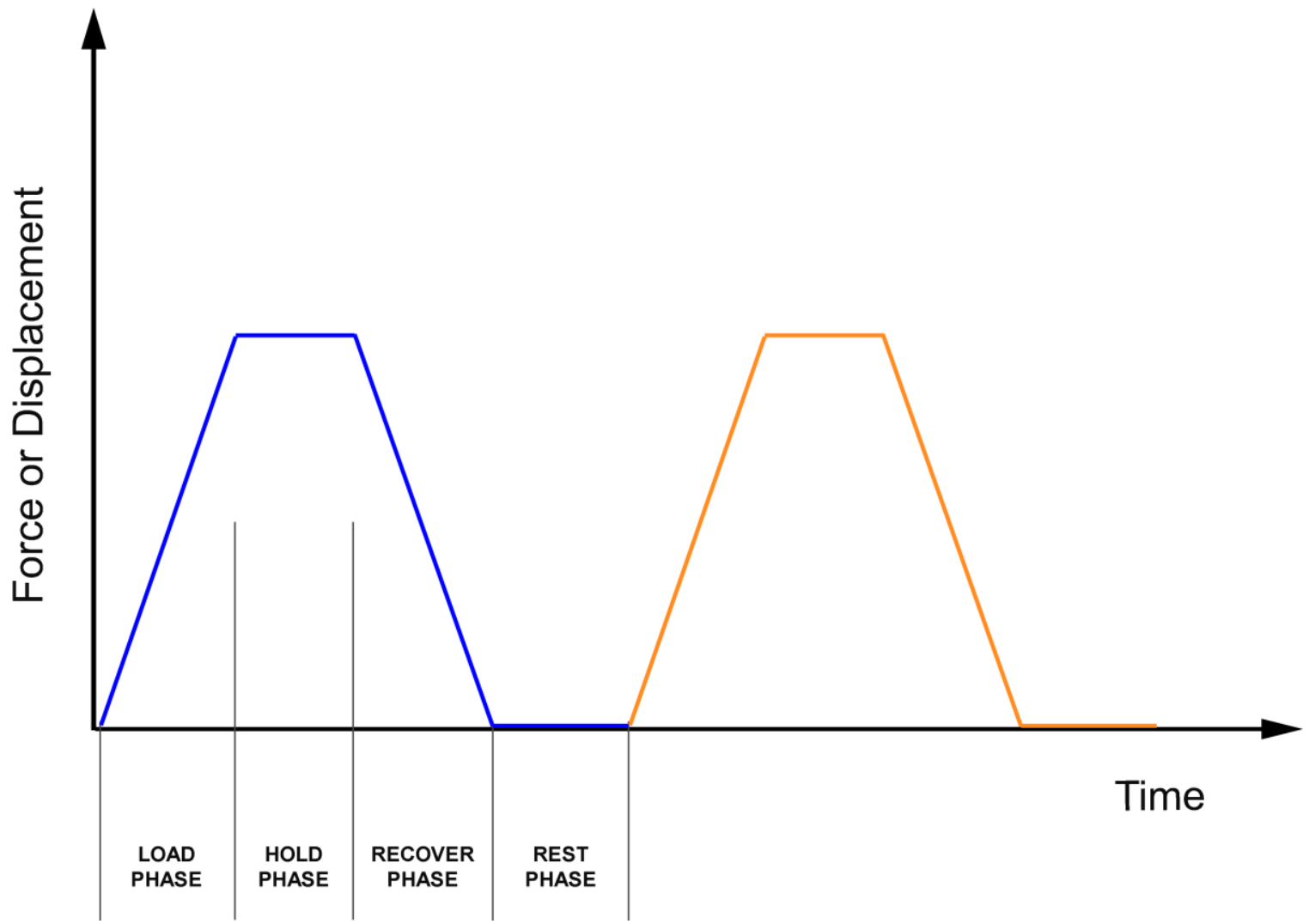

Figure 22. Test sequence showing the load, hold, recover, and rest phases using the CellScale Microsquisher. Adapted from [CellScale. MicroSquisher Micro-scale TensionCompression Test System]. ${ }^{178}$

The stiffness values may then be calculated based on a modified Hertz model ${ }^{179,180}$ using the following equations:

$$
\begin{gathered}
\emptyset=\cos ^{-1}\left[\frac{R-\delta}{R}\right] \\
a=(R-\delta) \tan \phi f(a)=\frac{2(1+v) R^{2}}{\left(a^{2}+4 R^{2}\right)^{3 / 2}}+\frac{1-v^{2}}{\left(a^{2}+4 R^{2}\right)^{1 / 2}} \\
E=\frac{3\left(1-v^{2}\right) F}{(4 \delta a)}-\frac{f(a) F}{\pi \delta}
\end{gathered}
$$


M.Sc. Thesis - C. Shamshoom - McMaster University- Chemistry and Chemical Biology

Where $\mathrm{F}$ is the applied force, $\mathrm{R}$ is the sphere radius, $\delta$ is the displacement, $v$ is the Poisson's ratio (0.5) and E is the Young's Modulus. The stiffness values are calculated using force and displacement values at an indentation of $10 \%$ of the indenter radius. ${ }^{179}$

To test the mechanical properties of the organogels, a compression probe was constructed by gluing a $3 \mathrm{~mm}$ diameter stainless steel ball to a $0.3048 \mu \mathrm{m}$ diameter cantilever. The organogel samples were prepared inside a modified $1 \mathrm{~mL}$ polypropylene syringe and allowed to gel for 30 minutes before extruding into a round Teflon mold (4 $\mathrm{mm}$ diameter and $3 \mathrm{~mm}$ deep) for mechanical testing. Native organogels were prepared by combining equimolar ratios of PF-Pillar to $\mathrm{PEG}_{600}(\mathrm{CN})_{2}$ in $50 \mu \mathrm{L}$ of 1,2-dichlorobenzene. Hybridized gels were prepared by mixing equimolar ratios of PF-Pillar to $\mathrm{PEG}_{600}-(\mathrm{CN})_{2}$ in $50 \mu \mathrm{L}$ of PF-Pillar-SWNT in 1,2-dichlorobenzene.

The organogel samples were indented using the MicroSquisher with a $3 \mathrm{~mm}$ stainless steel ball fixed to the end of the cantilever. The ball was indented into the gel by more than $0.3 \mathrm{~mm}$ in 60 seconds while collecting data at $1 \mathrm{~Hz}$. However, during the load phase, the indenter became stuck to the tacky gel, resulting in failure of the test sequence with no useful data obtained. Attempts to reduce the stickiness of the gel involved addition of a drop of silicone oil to the gel surface in order to prevent the indenter from sticking to the gel. It was found that addition of silicone oil did not mitigate the problem, and the test sequence failure ensued. Another attempt involved reduction of the contact time during the load phase to minimize the negative effects due the gel's texture. However, the indenter remained stuck to the organogel samples and the issues of the load phase could not be mitigated. 
M.Sc. Thesis - C. Shamshoom - McMaster University- Chemistry and Chemical Biology

\subsection{Mechanical Testing of Organogels using a Home-built Apparatus}

Given the drawbacks outlined for the CellScale Microsquisher, the mechanical properties of the organogels were measured by alternative means. A home-built apparatus was utilized (according to literature procedures ${ }^{181}$ ) to determine the Young's Modulus of the PF-Pillar and PF-Pillar-SWNT organogels. The instrument measures the contact mechanics between a glass hemisphere and an elastic organogel. This relationship can be described by Hertzian theory using equation 3:

$$
F=\frac{4 E R^{1 / 2}}{\left(1-v^{2}\right)} d^{3 / 2}
$$

Where $F$ is force $(\mathrm{N}), d$ is deformation $(\mathrm{mm}), R$ is radius of the glass hemispherical indenter $(\mathrm{mm}), v$ is the Poisson's ratio and $\mathrm{E}$ is the Young's modulus $(\mathrm{kPa})$ of the elastic substrate. ${ }^{182}$ Each organogel was investigated using a compression method with a hemispherical indenter consisting of a glass melting point tube (VWR) with a hemispherical end $(r=0.83$ $\mathrm{mm}$ ) attached to a force transducer (Transducer Techniques, GSO series, $10 \mathrm{~g}$ full scale). The vertical position of the glass indenter was controlled with a servo motor, which contacts and indents the gel at a constant speed. The force transducer is then able to measure the force applied by the gel relative to time and vertical position of the indenter. This was repeated in triplicate at different positions for each organogel. The Young's modulus was obtained using the equation described by Hertzian theory, rearranged to isolate Young's modulus as the slope when plotting the measured $F(d)$. Poisson's ratio is assumed to be $v$ $=0.45$, based on polymer gels having similar mechanical properties to elastic, rubber-like materials. $^{183,184}$ 
M.Sc. Thesis - C. Shamshoom - McMaster University- Chemistry and Chemical Biology

Mechanical testing of the PF-Pillar and PF-Pillar-SWNT organogels revealed Young's moduli of $6.0 \pm 0.2$ and $5.8 \pm 1.3 \mathrm{kPa}$, respectively. We hypothesize that the wt. $\%$ of SWNT in the PF-Pillar-SWNT organogel may be too low to observe substantial differences in mechanical properties. Overall, we demonstrate that supramolecular organogels incorporating PF-Pillar-coated SWNTs can be produced, and that gelation is unaffected by the presence of SWNTs.

\subsection{Summary}

We prepared a SWNT-containing organogel by crosslinking a PF-Pillar enriched dispersion with $\mathrm{PEG}_{600}-(\mathrm{CN})_{2}$ in THF. The initial mechanical testing of organogels in THF proved to be problematic, as rapid evaporation of the solvent led to inconsistent data. However, we found that employing a solvent exchange of PF-Pillar-SWNT dispersions in THF to 1,2-dichlorobenzene mitigated the problem of rapid solvent evaporation in the resultant gels. Utilising both Raman spectroscopy and PL mapping, we determined that solvent exchange methods did not increase bundling of the SWNTs in our dispersion. The mechanical testing of organogel samples was conducted using a home-built apparatus where it was found that the presence of SWNTs does not affect the Young's moduli of the gel. 
M.Sc. Thesis - C. Shamshoom - McMaster University- Chemistry and Chemical Biology

\section{Chapter 4: Conclusions}

\subsection{Overall Conclusions}

Single-walled carbon nanotubes are interesting allotropes of carbon that have unique structural, mechanical, optical and electronic properties. Control of single-walled carbon nanotube dispersion properties is of substantial interest to the scientific community. In this work, we sought to investigate the effect of a cavitand, the pillar[5]arene, on the dispersion properties of a polymer-nanotube complex. The electron-rich pillar[5]arene host is capable of forming inclusion complexes with electron-poor guests such as alkyl nitriles. Here, we demonstrate that a macrocycle-containing polyfluorene derivative can produce unusually concentrated polymer-SWNT dispersions. Utilizing the host-guest capability of pillar[5]arene, the polymer-nanotube complex was incorporated into a supramolecular organogel.

The synthesis of the pillar[5] arene-containing polyfluorene derivative was outlined in Chapter 2, where the quantitative alkylation of polyfluorene with mono-de-ethylated pillar[5]arene was confirmed by ${ }^{1} \mathrm{H}-\mathrm{NMR}$ spectroscopy. We show that the facile modification of a polyfluorene backbone with a pillar[5] arene macrocycle has a significant impact on the polymer-SWNT dispersion properties. As evidenced by UV-Vis-NIR spectroscopy, the dispersion post-functionalization with a macrocycle was substantially more concentrated than the corresponding dispersion without the cavitand. It was found that the polyfluorene derivative improved the concentration of SWNTs $\sim 30$-fold, from 20 to $600 \mu \mathrm{g} \cdot \mathrm{mL}^{-1}$, as determined by TGA. Using Raman and fluorescence spectroscopy, it was determined that the initial polyfluorene derivative preferentially dispersed sc-SWNT 
M.Sc. Thesis - C. Shamshoom - McMaster University- Chemistry and Chemical Biology

species, while the pillar[5]arene-decorated polyfluorene produced dispersions containing both sc- and m-SWNTs. The formation of an inclusion complex between pillar[5]arene and 1,6-dicyanohexane was confirmed in the presence of the macrocycle-containing polymerSWNT complex.

In Chapter 3, the host-guest properties of the macrocycle-containing polymerSWNT complex were further explored. Initially, we envisioned a crosslinked network of the pillar moieties with the alkyl nitrile side chains of a polyfluorene to form a "zipperlike" organogel. However, after several unsuccessful attempts to synthesize the akyl nitrilefunctionalized polymer, we prepared a homobifunctional PEG polymer with terminal alkyl nitriles to act as a crosslinker. The polymer-SWNT dispersion was successfully incorporated into supramolecular organogels. The mechanical testing of organogel samples was conducted using a home-built apparatus where it was found that the presence of SWNTs does not affect the Young's moduli of the gel.

The most significant contribution from this thesis includes the effect a macrocyclefunctionalized conjugated polymer on the dispersion of carbon nanotubes. It was found that incorporation of pillar[5] arene moieties in the side chains of polyfluorene had a significant impact on the populations of semiconducting and metallic nanotubes dispersed. This work shows that simple and facile incorporation of bulky pillar[5]arene structures in the side chains impart steric stabilization to the colloidal polymer-nanotube dispersion, resulting in a dispersion enriched with SWNTs. Thus, a simple modification of the conjugated polymer backbone can have a profound effect on the properties of the polymer-nanotube sample. In addition, we found that post-polymerization functionalization with the macrocycle yielded 
M.Sc. Thesis - C. Shamshoom - McMaster University- Chemistry and Chemical Biology

concentrated polymer-SWNT dispersions. Therefore, we were able to investigate the hostguest properties of a polymer-SWNT complex using ${ }^{1} \mathrm{H}-\mathrm{NMR}$ spectroscopy.

\subsection{Recommendations}

The work presented in this thesis strictly involved the incorporation of pillar[5]arenes in the side chains of polyfluorene for the non-covalent functionalization of carbon nanotubes. While there are a few examples of calixarenes ${ }^{185-187}$ used in the dispersion of SWNTs, the incorporation of other cavitands in conjugated polymer-SWNT complexes remain largely unexplored. It would be interesting to investigate the effect of other macrocycles on the dispersion properties of a polymer-nanotube complex. For instance, one would compare the relative SWNT concentration and nanotube species dispersed before and after cavitand-functionalization of a conjugated polymer. The results of these studies may be compared to those presented in this thesis to gain further understanding of the effects of macrocycles on the dispersion properties of conjugated polymer-SWNT complexes. 
M.Sc. Thesis - C. Shamshoom - McMaster University- Chemistry and Chemical Biology

\section{References}

(1) Krätschmer, W.; Lamb, L. D.; Fostiropoulos, K.; Huffman, D. R. Nature 1990, 347 (6291), 354-358.

(2) Iijima, S. Nature 1991, 354 (6348), 56-58.

(3) Tasis, D.; Tagmatarchis, N.; Bianco, A.; Prato, M. Chem. Rev. 2006, 106 (3), 1105-1136.

(4) Park, S.; Vosguerichian, M.; Bao, Z. Nanoscale 2013, 5 (5), 1727.

(5) Terrones, M. Annu. Rev. Mater.Res 2003, 33, 419-501.

(6) Ajayan, P. M. Chem. Rev. 1999, 99 (7), 1787-1800.

(7) Saito, R.; Dresselhaus, G.; Dresselhaus, M. Physical Properties of Carbon Nanotubes; 1998.

(8) Coleman, J. N.; Khan, U.; Blau, W. J.; Gun'ko, Y. K. Carbon 2006, 44 (9), $1624-$ 1652.

(9) Zhang, Q.; Huang, J.-Q.; Qian, W.-Z.; Zhang, Y.-Y.; Wei, F. Small 2013, 9 (8), $1237-1265$.

(10) O’Connell, M. J.; Bachilo, S. M.; Huffman, C. B.; Moore, V. C.; Strano, M. S.; Haroz, E. H.; Rialon, K. L.; Boul, P. J.; Noon, W. H.; Kittrell, C.; Ma, J.; Hauge, R. H.; Weisman, R. B.; Smalley, R. E. Science 2002, 297 (5581), 593-596.

(11) Bachilo, S. M.; Strano, M. S.; Kittrell, C.; Hauge, R. H.; Smalley, R. E.; Weisman, R. B. Science 2002, 298 (5602), 2361-2366.

(12) Kataura, H.; Kumazawa, Y.; Maniwa, Y.; Umezu, I.; Suzuki, S.; Ohtsuka, Y.; Achiba, Y. Synth. Met. 1999, 103 (1-3), 2555-2558. 
M.Sc. Thesis - C. Shamshoom - McMaster University- Chemistry and Chemical Biology

(13) Rice, N. A. Separation of Single-Walled Carbon Nanotubes By Electronic Type Using Conjugated Polymers, McMaster University, 2015.

(14) Bhushan, B. Springer Handbook of Nanotechnology; Bhushan, B., Ed.; Springer US, 2004.

(15) Balasubramanian, K.; Burghard, M. Small 2005, 1 (2), 180-192.

(16) Chen, F.; Wang, B.; Chen, Y.; Li, L. J. Nano Lett. 2007, 7 (10), 3013-3017.

(17) Rafique, M. M. A.; Iqbal, J. J. Encapsulation Adsorpt. Sci. 2011, 1, 29-34.

(18) Guo, T.; Nikolaev, P.; Thess, A.; Colbert, D. T.; Smalley, R. E. Chem. Phys. Lett. 1995, $243(1-2), 49-54$.

(19) Scott, C. D.; Arepalli, S.; Nikolaev, P.; Smalley, R. E. Appl. Phys. A Mater. Sci. Process. 2001, 72 (5), 573-580.

(20) Li, W. Z.; Xie, S. S.; Qian, L. X.; Chang, B. H.; Zou, B. S.; Zhou, W. Y.; Zhao, R. A.; Wang, G. Science 1996, 274 (5293), 1701-1703.

(21) Chiang, I. W.; Brinson, B. E.; Huang, A. Y.; Willis, P. A.; Bronikowski, M. J.; Margrave, J. L.; Smalley, R. E.; Hauge, R. H. J. Phys. Chem. B 2001, 105 (35), $8297-8301$.

(22) Resasco, D. E.; Alvarez, W. E.; Pompeo, F.; Balzano, L.; Herrera, J. E.; Kitiyanan, B.; Borgna, A. J. Nanoparticle Res. 2002, 4 (1/2), 131-136.

(23) Nikolaev, P.; Bronikowski, M. J.; Bradley, R. K.; Rohmund, F.; Colbert, D. T.; Smith, K. .; Smalley, R. E. Chem. Phys. Lett. 1999, 313 (1-2), 91-97.

(24) Kong, J.; Cassell, A. M.; Dai, H. Chem. Phys. Lett. 1998, 292 (4-6), 567-574.

(25) Journet, C.; Maser, W. K.; Bernier, P.; Loiseau, A.; de la Chapelle, M. L.; Lefrant, 
M.Sc. Thesis - C. Shamshoom - McMaster University- Chemistry and Chemical Biology

S.; Deniard, P.; Lee, R.; Fischer, J. E. Nature 1997, 388 (6644), 756-758.

(26) Guo, T.; Nikolaev, P.; Thess, A.; Colbert, D. T.; Smalley, R. E. Chem. Phys. Lett. 1995, 243 (1-2), 49-54.

(27) Kim, K. S.; Cota-Sanchez, G.; Kingston, C. T.; Imris, M.; Simard, B.; Soucy, G. J. Phys. D. Appl. Phys. 2007, 40 (8), 2375-2387.

(28) Campidelli, S.; Klumpp, C.; Bianco, A.; Guldi, D. M.; Prato, M. J. Phys. Org. Chem. 2006, 19 (8-9), 531-539.

(29) Britz, D. A.; Khlobystov, A. N. Chem. Soc. Rev. 2006, 35 (7), 637.

(30) Hirsch, A. Angew. Chemie - Int. Ed. 2002, 41 (11), 1853-1859.

(31) Li, H.; Cheng, F.; Duft, A. M.; Adronov, A. J. Am. Chem. Soc. 2005, 127 (41), $14518-14524$.

(32) Liu, J.; Rinzler, A. G.; Dai, H.; Hafner, J. H.; Kelley Bradley, R.; Boul, P. J.; Lu, A.; Iverson, T.; Shelimov, K.; Huffman, C. B.; Rodriguez-Macias, F.; Shon, Y. S.; Lee, T. R.; Colbert, D. T.; Smalley, R. E. Science 1998, 280 (5367), 1253-1256.

(33) Gavrel, G.; Jousselme, B.; Filoramo, A.; Campidelli, S. Springer, Berlin, Heidelberg, 2013; pp 95-126.

(34) Bilalis, P.; Katsigiannopoulos, D.; Avgeropoulos, A.; Sakellariou, G. RSC Adv. 2014, 4 (6), 2911-2934.

(35) Gong, X.; Liu, J.; Baskaran, S.; Voise, R. D.; Young, J. S. Chem. Mater. 2000, 12 (4), 1049-1052.

(36) Islam, M. F.; Rojas, E.; Bergey, D. M.; Johnson, A. T.; Yodh, A. G. Nano Lett. 2003, 3 (2), 269-273. 
M.Sc. Thesis - C. Shamshoom - McMaster University- Chemistry and Chemical Biology

(37) Moore, V. C.; Strano, M. S.; Haroz, E. H.; Hauge, R. H.; Smalley, R. E.; Schmidt, J.; Talmon, Y. Nano Lett. 2003, 3 (10), 1379-1382.

(38) Chen, R. J.; Zhang, Y.; Wang, D.; Dai, H. Science 2001, 123 (16), 3838-3839.

(39) Tomonari, Y.; Murakami, H.; Nakashima, N. Chem. - A Eur. J. 2006, 12 (15), $4027-4034$.

(40) Yang, K.; Zhu, L.; Xing, B. Environ. Sci. Technol. 2006, 40 (6), 1855-1861.

(41) Nish, A.; Hwang, J.-Y.; Doig, J.; Nicholas, R. J. Nat. Nanotechnol. 2007, 2 (10), 640.

(42) Star, A.; Stoddart, J. F.; Steuerman, D.; Diehl, M.; Boukai, A.; Wong, E. W.; Yang, X.; Chung, S.-W.; Choi, H.; Heath, J. R. Angew. Chemie - Int. Ed. 2001, 40 (9), 1721-1725.

(43) Nish, A.; Hwang, J.-Y.; Doig, J.; Nicholas, R. J. Nat. Nanotechnol. 2007, 2 (10), 640-646.

(44) Heller, D. A.; Jeng, E. S.; Yeung, T.-K.; Martinez, B. M.; Moll, A. E.; Gastala, J. B.; Strano, M. S. Science 2006, 311 (5760), 508-511.

(45) Zheng, M.; Jagota, A.; Semke, E. D.; Diner, B. A.; Mclean, R. S.; Lustig, S. R.; Richardson, R. E.; Tassi, N. G. Nat. Mater. 2003, 2 (5), 338-342.

(46) Zheng, M.; Jagota, A.; Strano, M. S.; Santos, A. P.; Barone, P.; Chou, S. G.; Diner, B. A.; Dresselhaus, M. S.; McLean, R. S.; Onoa, G. B.; Samsonidze, G. G.; Semke, E. D.; Usrey, M.; Walls, D. J. Science 2003, 302 (5650), 1545-1548.

(47) Bradley, K.; Briman, M.; Star, A.; Gruner, G. Nano Lett. 2004, 4 (2), 253-256.

(48) Balavoine, F.; Schultz, P.; Richard, C.; Mallouh, V.; Ebbesen, T. W.; Mioskowski, 
M.Sc. Thesis - C. Shamshoom - McMaster University- Chemistry and Chemical Biology

C. Angew. Chemie - Int. Ed. 1999, 38 (13-14), 1912-1915.

(49) Guo, Z.; Sadler, P. J.; Tsang, S. C. Adv. Mater. 1998, 10 (9), 701-703.

(50) Moore, V. C.; Strano, M. S.; Haroz, E. H.; Hauge, R. H.; Smalley, R. E.; Schmidt, J.; Talmon, Y. Nano Lett. 2003, 3 (10), 1379-1382.

(51) Blanch, A. J.; Lenehan, C. E.; Quinton, J. S. J. Phys. Chem. B 2010, 114 (30), 9805-9811.

(52) Arnold, M. S.; Green, A. A.; Hulvat, J. F.; Stupp, S. I.; Hersam, M. C. Nat. Nano. 2006, 1 (1), 60 .

(53) Graham, J. M. Biological Centrifugation (The Basics); BIOS Scientific: Milton Park, 2001.

(54) Hersam, M. C. Nature Nanotechnology. Nature Publishing Group July 30, 2008, pp 387-394.

(55) Miyata, Y.; Yanagi, K.; Maniwa, Y.; Kataura, H. J. Phys. Chem. C 2008, 112 (10), 3591-3596.

(56) Crochet, J.; Clemens, M.; Hertel, T. J. Am. Chem. Soc. 2007, 129 (26), 8058-8059.

(57) Backes, C.; Hauke, F.; Schmidt, C. D.; Hirsch, A. Chem. Commun. 2009, 0 (19), $2643-2645$.

(58) Green, A. A.; Hersam, M. C. Nano Lett. 2008, 8 (5), 1417-1422.

(59) Ghosh, S.; Bachilo, S. M.; Weisman, R. B. Nat. Nanotechnol. 2010, 5 (6), 443450.

(60) Jung, S.; Cha, M.; Park, J.; Jeong, N.; Kim, G.; Park, C.; Ihm, J.; Lee, J. J. Am. Chem. Soc. 2010, 132 (32), 10964-10966. 
M.Sc. Thesis - C. Shamshoom - McMaster University- Chemistry and Chemical Biology

(61) Tu, X.; Manohar, S.; Jagota, A.; Zheng, M. Nature 2009, 460 (7252), 250-253.

(62) Woo, H. S.; Czerw, R.; Webster, S.; Carroll, D. L.; Ballato, J.; Strevens, A. E.; O’Brien, D.; Blau, W. J. Appl. Phys. Lett. 2000, 77 (9), 1393-1395.

(63) Steuerman, D. W.; Star, A.; Narizzano, R.; Choi, H.; Ries, R. S.; Nicolini, C.; Stoddart, J. F.; Heath, J. R. J. Phys. Chem. B 2002, 106 (12), 3124-3130.

(64) Chen, J.; Liu, H.; Weimer, W. A.; Halls, M. D.; Waldeck, D. H.; Walker, G. C. J. Am. Chem. Soc. 2002, 124 (31), 9034-9035.

(65) Bilalis, P.; Katsigiannopoulos, D.; Avgeropoulos, A.; Sakellariou, G. RSC Adv. 2014, 4 (6), 2911-2934.

(66) Gomulya, W.; Gaoa, J.; Loib, M. A. Eur. Phys. J. B 2013, 86 (10), 404.

(67) Zhang, D.; Yang, J.; Li, Y. Small 2013, 9 (8), 1284-1304.

(68) Cheng, F.; Imin, P.; Maunders, C.; Botton, G.; Adronov, A. Macromolecules 2008, 41 (7), 2304-2308.

(69) O’Connell, M. J.; Bachilo, S. M.; Huffman, C. B.; Moore, V. C.; Strano, M. S.; Haroz, E. H.; Rialon, K. L.; Boul, P. J.; Noon, W. H.; Kittrell, C.; Ma, J.; Hauge, R. H.; Weisman, R. B.; Smalley, R. E. Science 2002, 297 (5581), 593-596.

(70) O’Connell, M. J.; Bachilo, S. M.; Huffman, C. B.; Moore, V. C.; Strano, M. S.; Haroz, E. H.; Rialon, K. L.; Boul, P. J.; Noon, W. H.; Kittrell, C.; Ma, J.; Hauge, R. H.; Weisman, R. B.; Smalley, R. E. Science 2002, 297 (5581), 593-596.

(71) Strano, M. S.; Dyke, C. A.; Usrey, M. L.; Barone, P. W.; Allen, M. J.; Shan, H.; Kittrell, C.; Hauge, R. H.; Tour, J. M.; Smalley, R. E. Science 2003, 301 (2003), $1519-1522$. 
M.Sc. Thesis - C. Shamshoom - McMaster University- Chemistry and Chemical Biology

(72) Luo, Z.; Pfefferle, L. D.; Haller, G. L.; Papadimitrakopoulos, F. J. Am. Chem. Soc. 2006, $128(48), 15511-15516$.

(73) Bachilo, S. M.; Strano, M. S.; Kittrell, C.; Hauge, R. H.; Smalley, R. E.; Weisman, R. B. Science 2002, 298 (5602), 2361-2366.

(74) R. Bruce Weisman*; Bachilo, S. M. Nano Lett. 2003, 3 (9), 1235-1238.

(75) Kataura, H.; Kumazawa, Y.; Maniwa, Y.; Umezu, I.; Suzuki, S.; Ohtsuka, Y.; Achiba, Y. Synth. Met. 1999, 103 (1-3), 2555-2558.

(76) Ohno, Y.; Maruyama, S.; Mizutani, T. 1998, 109-123.

(77) Michel, T.; Paillet, M.; Nakabayashi, D.; Picher, M.; Jourdain, V.; Meyer, J. C.; Zahab, A. A.; Sauvajol, J. L. Phys. Rev. B - Condens. Matter Mater. Phys. 2009, $80(24), 245416$.

(78) Fantini, C.; Jorio, A.; Santos, A. P.; Peressinotto, V. S. T.; Pimenta, M. A. Chem. Phys. Lett. 2007, 439 (1-3), 138-142.

(79) Dresselhaus, M. S.; Dresselhaus, G.; Jorio, A.; Souza Filho, A. G.; Pimenta, M. A.; Saito, R. Acc. Chem. Res. 2002, 35 (12), 1070-1078.

(80) Sato, K.; Saito, R.; Jiang, J.; Dresselhaus, G.; Dresselhaus, M. S. Phys. Rev. B Condens. Matter Mater. Phys. 2007, 76 (19), 195446.

(81) Nugraha, A. R. T.; Saito, R.; Sato, K.; Araujo, P. T.; Jorio, A.; Dresselhaus, M. S. Appl. Phys. Lett. 2010, 97 (9), 091905.

(82) Rao, A. M.; Richter, E.; Bandow, S.; Chase, B.; Eklund, P. C.; Williams, K. A.; Fang, S.; Subbaswamy, K. R.; Menon, M.; Thess, A.; Smalley, R. E.; Dresselhaus, G.; Dresselhaus, M. S. Science 1997, 275 (5297), 187-190. 
M.Sc. Thesis - C. Shamshoom - McMaster University- Chemistry and Chemical Biology

(83) Dresselhaus, M. S.; Dresselhaus, G.; Saito, R.; Jorio, A. Phys. Rep. 2005, 409 (2), 47.

(84) Garrett, M.; Ivanov, I. N.; Geohegan, D.; Hu, B. Carbon 2013, 64, 1-5.

(85) Park, T. J.; Banerjee, S.; Hemraj-Benny, T.; Wong, S. S. J. Mater. Chem. 2006, 16 (2), 141-154.

(86) Brown, S. D. M.; Jorio, A.; Corio, P.; Dresselhaus, M. S.; Dresselhaus, G.; Saito, R.; Kneipp, K. Phys. Rev. B - Condens. Matter Mater. Phys. 2001, 63 (15), 155414.

(87) Lehn, J. -M. Angew. Chemie - Int. Ed. 1988, 27 (1), 89-112.

(88) Cram, D. J. Angewandte Chemie International Edition in English. WileyBlackwell August 1, 1988, pp 1009-1020.

(89) Pedersen, C. J. Angewandte Chemie International Edition in English. WileyBlackwell August 1, 1988, pp 1021-1027.

(90) Steed, K.; Turner, D.; Wallace, K. Core concepts in supramolecular chemistry and nanochemistry; John Wiley \& Sons, Ltd, 2007.

(91) Chen, G.; Jiang, M. Chemical Society Reviews. Royal Society of Chemistry April 18, 2011, pp 2254-2266.

(92) Szejtli, J. Chem. Rev. 1998, 98 (5), 1743-1754.

(93) Davis, M. E.; Brewster, M. E. Nature Reviews Drug Discovery. Nature Publishing Group December 1, 2004, pp 1023-1035.

(94) Guo, D. S.; Liu, Y. Chemical Society Reviews. Royal Society of Chemistry August 20, 2012, pp 5907-5921. 
M.Sc. Thesis - C. Shamshoom - McMaster University- Chemistry and Chemical Biology

(95) Kim, J. S.; Quang, D. T. Chemical Reviews. American Chemical Society 2007, pp $3780-3799$.

(96) Rebek Jr., J. Chem. Commun. 2000, 0 (8), 637-643.

(97) Kim, K. Chemical Society Reviews. Royal Society of Chemistry March 18, 2002, pp 96-107.

(98) Assaf, K. I.; Nau, W. M. Chemical Society Reviews. Royal Society of Chemistry December 22, 2015, pp 394-418.

(99) Kim, K.; Selvapalam, N.; Ko, Y. H.; Park, K. M.; Kim, D.; Kim, J. Chem. Soc. Rev. 2007, 36 (2), 267-279.

(100) Ogoshi, T.; Shiga, R.; Hashizume, M.; Yamagishi, T. A. Chem. Commun. 2011, 47 (24), 6927-6929.

(101) Ogoshi, T.; Demachi, K.; Kitajima, K.; Yamagishi, T. A. Chem. Commun. 2011, 47 (25), 7164-7166.

(102) Ogoshi, T.; Kitajima, K.; Aoki, T.; Fujinami, S.; Yamagishi, T. A.; Nakamoto, Y. J. Org. Chem. 2010, 75 (10), 3268-3273.

(103) Ogoshi, T.; Kanai, S.; Fujinami, S.; Yamagishi, T.-A.; Nakamoto, Y. J. Am. Chem. Soc. 2008, 130, 5022-5023.

(104) Strutt, N. L.; Zhang, H.; Giesener, M. A.; Lei, J.; Stoddart, J. F. Chem. Commun. 2012, 48 (11), 1647-1649.

(105) Ogoshi, T.; Hashizume, M.; Yamagishi, T. A.; Nakamoto, Y. Chem. Commun. 2010, 46 (21), 3708-3710.

(106) Ogoshi, T.; Nishida, Y.; Yamagishi, T. A.; Nakamoto, Y. Macromolecules 2010, 
M.Sc. Thesis - C. Shamshoom - McMaster University- Chemistry and Chemical Biology

$43(7), 3145-3147$.

(107) Deng, H.; Shu, X.; Hu, X.; Li, J.; Jia, X.; Li, C. Tetrahedron Lett. 2012, 53 (34), 4609-4612.

(108) Strutt, N. L.; Forgan, R. S.; Spruell, J. M.; Botros, Y. Y.; Stoddart, J. F. J. Am. Chem. Soc. 2011, 133 (15), 5668-5671.

(109) Shu, X.; Chen, S.; Li, J.; Chen, Z.; Weng, L.; Jia, X.; Li, C. Chem. Commun. 2012, 48 (24), 2967.

(110) Li, Z. Y.; Zhang, Y.; Zhang, C. W.; Chen, L. J.; Wang, C.; Tan, H.; Yu, Y.; Li, X.; Yang, H. B. J. Am. Chem. Soc. 2014, 136 (24), 8577-8589.

(111) Ye, F.; Wei, R.; Wang, L.; Meier, H.; Cao, D. RSC Adv. 2016, 6 (92), 89810 89814.

(112) Ogoshi, T.; Nishida, Y.; Yamagishi, T.; Nakamoto, Y. Macromolecules 2010, 43 (7), 3145-3147.

(113) Ogoshi, T.; Nishida, Y.; Yamagishi, T.; Nakamoto, Y. Macromolecules 2010, 43 (17), 7068-7072.

(114) Ogoshi, T.; Hasegawa, Y.; Aoki, T.; Ishimori, Y.; Inagi, S.; Yamagishi, T. Macromolecules 2011, 44 (19), 7639-7644.

(115) Sun, S.; Hu, X. Y.; Chen, D.; Shi, J.; Dong, Y.; Lin, C.; Pan, Y.; Wang, L. Polym. Chem. 2013, 4 (7), 2224-2229.

(116) Wang, P.; Yao, Y.; Xue, M. Chem. Commun. 2014, 50 (39), 5064-5067.

(117) Ogoshi, T.; Aoki, T.; Ueda, S.; Tamura, Y.; Yamagishi, T. Chem. Commun. 2014, $50(50), 6607-6609$. 
M.Sc. Thesis - C. Shamshoom - McMaster University- Chemistry and Chemical Biology

(118) Chang, J.; Zhao, Q.; Kang, L.; Li, H.; Xie, M.; Liao, X. Macromolecules 2016, 49 (7), 2814-2820.

(119) Gribble, G. W.; Nutaitis, C. F. Tetrahedron Lett. 1985, 26 (49), 6023-6026.

(120) Gutsche, C. D. Calixarenes Revisited; 1998.

(121) Patrick, T. B.; Egan, P. A. Journal of Organic Chemistry. 1977, pp 382-383.

(122) Gutsche, C. D.; Muthukrishnan, R. J. Org. Chem. 1978, 43 (25), 4905-4906.

(123) Rowan, S. J.; Cantrill, S. J.; Cousins, G. R. L.; Sanders, J. K. M.; Stoddart, J. F. Angew. Chemie - Int. Ed. 2002, 41 (6), 898-952.

(124) Alexander, V. Chem. Rev. 1995, 95, 273-342.

(125) Holler, M.; Allenbach, N.; Sonet, J.; Nierengarten, J. F. Chem. Commun. 2012, 48 (20), 2576-2578.

(126) Cragg, P. J.; Sharma, K. Chemical Society Reviews. Royal Society of Chemistry January 4, 2012, pp 597-607.

(127) Strutt, N. L.; Zhang, H.; Schneebeli, S. T.; Stoddart, J. F. Acc. Chem. Res. 2014, 47 (8), 2631-2642.

(128) Strutt, N. L.; Zhang, H.; Giesener, M. A.; Lei, J.; Stoddart, J. F. Chem. Commun. 2012, 48 (11), 1647-1649.

(129) Zhang, H.; Strutt, N. L.; Stoll, R. S.; Li, H.; Zhu, Z.; Stoddart, J. F. Chem. Commun. 2011, 47 (41), 11420-11422.

(130) Zhang, H.; Ma, X.; Guo, J.; Nguyen, K. T.; Zhang, Q.; Wang, X. J.; Yan, H.; Zhu, L.; Zhao, Y. RSC Adv. 2013, 3 (2), 368-371.

(131) Ogoshi, T.; Demachi, K.; Kitajima, K.; Yamagishi, T. A. Chem. Commun. 2011, 
M.Sc. Thesis - C. Shamshoom - McMaster University- Chemistry and Chemical Biology

47 (25), 7164-7166.

(132) Ogoshi, T.; Demachi, K.; Kitajima, K.; Yamagishi, T. Chem. Commun. 2011, 47 (37), 10290.

(133) Chen, Y.; He, M.; Li, B.; Wang, L.; Meier, H.; Cao, D. RSC Adv. 2013, 3 (44), 21405-21408.

(134) Ogoshi, T. Pillararenes; Ogoshi, T., Ed.; Royal Society of Chemistry, 2016.

(135) Ogoshi, T.; Kitajima, K.; Aoki, T.; Yamagishi, T. A.; Nakamoto, Y. J. Phys. Chem. Lett. 2010, 1 (5), 817-821.

(136) Deng, H.; Shu, X.; Hu, X.; Li, J.; Jia, X.; Li, C. Tetrahedron Lett. 2012, 53 (34), 4609-4612.

(137) Shu, X.; Chen, S.; Li, J.; Chen, Z.; Weng, L.; Jia, X.; Li, C. Chem. Commun. 2012, 48 (24), 2967-2969.

(138) Shu, X.; Fan, J.; Li, J.; Wang, X.; Chen, W.; Jia, X.; Li, C. Org. Biomol. Chem. 2012, 10 (17), 3393-3397.

(139) Thordarson, P. Chemical Society Reviews. 2011, pp 1305-1323.

(140) Liang, S.; Zhao, Y.; Adronov, A. J. Am. Chem. Soc. 2014, 136 (3), 970-977.

(141) Pochorovski, I.; Wang, H.; Feldblyum, J. I.; Zhang, X.; Antaris, A. L.; Bao, Z. J. Am. Chem. Soc. 2015, 137 (13), 4328-4331.

(142) Lei, T.; Pochorovski, I.; Bao, Z. Acc. Chem. Res. 2017, 50 (4), 1096-1104.

(143) Lei, T.; Chen, X.; Pitner, G.; Wong, H.-S. P.; Bao, Z. J. Am. Chem. Soc. 2016, 138 (3), 802-805.

(144) Toshimitsu, F.; Nakashima, N. Nat. Commun. 2014, 5, 5041. 
M.Sc. Thesis - C. Shamshoom - McMaster University- Chemistry and Chemical Biology

(145) Fong, D.; Andrews, G. M.; McNelles, S. A.; Adronov, A. Polym. Chem. 2018, 9 (35), 4460-4467.

(146) Fong, D.; Andrews, G. M.; Adronov, A. Polym. Chem. 2018, 9 (21), 2873-2879.

(147) Fong, D.; Yeung, J.; McNelles, S. A.; Adronov, A. Macromolecules 2018, 51 (3), $755-762$.

(148) Driggers, E. M.; Hale, S. P.; Lee, J.; Terrett, N. K. Nature Reviews Drug Discovery. Nature Publishing Group July 1, 2008, pp 608-624.

(149) Heinis, C. Nat. Chem. Biol. 2014, 10 (9), 696-698.

(150) Marsault, E.; Peterson, M. L. J. Med. Chem. 2011, 54 (7), 1961-2004.

(151) Ogoshi, T.; Harada, A. Sensors 2008, 8 (8), 4961-4982.

(152) Iyoda, M.; Yamakawa, J.; Rahman, M. J. Angewandte Chemie - International Edition. Wiley-Blackwell November 4, 2011, pp 10522-10553.

(153) Chen, Z.; Wang, Q.; Wu, X.; Li, Z.; Jiang, Y. B. Chem. Soc. Rev. 2015, 44 (13), 4249-4263.

(154) Guo, D.-S.; Liu, Y. Chem. Soc. Rev. 2012, 41 (18), 5907.

(155) Ogoshi, T.; Demachi, K.; Kitajima, K.; Yamagishi, T. A. Chem. Commun. 2011, 47 (25), 7164-7166.

(156) Ogoshi, T.; Kitajima, K.; Aoki, T.; Fujinami, S.; Yamagishi, T. A.; Nakamoto, Y. J. Org. Chem. 2010, 75 (10), 3268-3273.

(157) Chang, J.; Zhao, Q.; Kang, L.; Li, H.; Xie, M.; Liao, X. Macromolecules 2016, 49 (7), 2814-2820.

(158) Rice, N. A.; Adronov, A. Macromolecules 2013, 46 (10), 3850-3860. 
M.Sc. Thesis - C. Shamshoom - McMaster University- Chemistry and Chemical Biology

(159) Fong, D.; Bodnaryk, W. J.; Rice, N. A.; Saem, S.; Moran-Mirabal, J. M.; Adronov, A. Chem. - A Eur. J. 2016, 22 (41), 14560-14566.

(160) Lemasson, F.; Berton, N.; Tittmann, J.; Hennrich, F.; Kappes, M. M.; Mayor, M. Macromolecules 2012, 45 (2), 713-722.

(161) Lemasson, F. A.; Strunk, T.; Gerstel, P.; Hennrich, F.; Lebedkin, S.; BarnerKowollik, C.; Wenzel, W.; Kappes, M. M.; Mayor, M. J. Am. Chem. Soc. 2011, $133(4), 652-655$.

(162) Strano, M. S.; Zheng, M.; Jagota, A.; Onoa, G. B.; Heller, D. A.; Barone, P. W.; Usrey, M. L. Nano Lett. 2004, 4 (4), 543-550.

(163) Strano, M. S.; Doorn, S. K.; Haroz, E. H.; Kittrell, C.; Hauge, R. H.; Smalley, R. E. Nano Lett. 2003, 3 (8), 1091-1096.

(164) Strano, M. S.; Dyke, C. A.; Usrey, M. L.; Barone, P. W.; Allen, M. J.; Shan, H.; Kittrell, C.; Hauge, R. H.; Tour, J. M.; Smalley, R. E. Science 2003, 301 (5639), $1519-1522$.

(165) Doorn, S. K.; Heller, D. A.; Barone, P. W.; Usrey, M. L.; Strano, M. S. Appl. Phys. A Mater. Sci. Process. 2004, 78 (8), 1147-1155.

(166) Kardelis, V.; Li, K.; Nierengarten, I.; Holler, M.; Nierengarten, J. F.; Adronov, A. Macromolecules 2017, 50 (23), 9144-9150.

(167) Chen, Q.; Zhu, L.; Zhao, C.; Wang, Q.; Zheng, J. Adv. Mater. 2013, 25 (30), $4171-4176$.

(168) Rong, Q.; Lei, W.; Chen, L.; Yin, Y.; Zhou, J.; Liu, M. Angew. Chemie - Int. Ed. 2017. 
M.Sc. Thesis - C. Shamshoom - McMaster University- Chemistry and Chemical Biology

(169) Coleman, J. N.; Khan, U.; Gun'ko, Y. K. Adv. Mater. 2006, 18 (6), 689-706.

(170) Cadek, M.; Coleman, J. N.; Ryan, K. P.; Nicolosi, V.; Bister, G.; Fonseca, A.; Nagy, J. B.; Szostak, K.; Béguin, F.; Blau, W. J. Nano Lett. 2004, 4 (2), 353-356.

(171) Baughman, R. H.; Zakhidov, A. A.; de Heer, W. A. Science 2002, 297 (5582), $787-792$.

(172) Coleman, J. N.; Khan, U.; Blau, W. J.; Gun'ko, Y. K. Carbon 2006, 44 (9), $1624-$ 1652.

(173) Ajayan, P. M.; Tour, J. M. Nature 2007, 447 (7148), 1066-1068.

(174) Christopherson, D. G.; L Cox, B. H. Appl. iMath. Soc. A 1948, 1 (165), 253-248.

(175) Treacy, M. M. J.; Ebbesen, T. W.; Gibson, J. M. Nature 1996, 381 (6584), 678680.

(176) Mastracchio, A. Phase-Transfer Catalysis http://chemlabs.princeton.edu/macmillan/wp-content/uploads/sites/6/AM_phasetransfer-catalysis.pdf (accessed Oct 1, 2017).

(177) Fong, D.; Bodnaryk, W. J.; Rice, N. A.; Saem, S.; Moran-Mirabal, J. M.; Adronov, A. Chem. - A Eur. J. 2016, 22 (41), 14560-14566.

(178) CellScale. MicroSquisher Micro-scale Tension-Compression Test System https://cellscale.com/wp-content/uploads/2016/11/MicroSquisher-User-Manualv3.5.pdf.

(179) Compression Testing of Soft Materials Compression of a Cylindrical Specimen http://cellscale.com/wp-content/uploads/2016/11/Compression-Testing-of-SoftMaterials.pdf (accessed Jan 10, 2018). 
M.Sc. Thesis - C. Shamshoom - McMaster University- Chemistry and Chemical Biology

(180) Kim, K.; Cheng, J.; Liu, Q.; Wu, X. Y.; Sun, Y. J. Biomed. Mater. Res. Part A 2010, $92 A(1), 103-113$.

(181) Hodgson, S. M.; Bakaic, E.; Stewart, S. A.; Hoare, T.; Adronov, A. Biomacromolecules 2016, 17 (3), 1093-1100.

(182) Long, R.; Hall, M. S.; Wu, M.; Hui, C.-Y. Biophys. J. 2011, 101 (3), 643-650.

(183) Anseth, K. S.; Bowman, C. N.; Brannon-Peppas, L. Biomaterials. Elsevier January 1, 1996, pp 1647-1657.

(184) Takigawa, T.; Morino, Y.; Urayama, K.; Masuda, T. Polym. Gels Networks 1996, $4(1), 1-5$.

(185) Ling, I.; Alias, Y.; Makha, M.; Raston, C. L. New J. Chem. 2009, 33 (7), 15831587.

(186) Dionisio, M.; Schnorr, J. M.; Michaelis, V. K.; Griffin, R. G.; Swager, T. M.;

Dalcanale, E. J. Am. Chem. Soc. 2012, 134 (15), 6540-6543.

(187) Lobach, A. S.; Ryzhkina, I. S.; Spitsina, N. G.; Obraztsova, E. D. In Physica Status Solidi (B) Basic Research; Wiley-Blackwell, 2007; Vol. 244, pp 4030-4034. 
M.Sc. Thesis - C. Shamshoom - McMaster University- Chemistry and Chemical Biology

\section{Appendix A: General Experimental}

All reagents were obtained from commercial sources and were used as received without further purification. Raw HiPCO SWNTs were purchased from NanoIntegris (batch \#HR27-104, 10 wt \% in anhydrous EtOH) and used without further purification. Flash chromatography was performed using an IntelliFlash 280 system from Analogix. Unless otherwise noted, compounds were monitored using a variable wavelength detector at $254 \mathrm{~nm}$. Solvent amounts used for gradient or isocratic elution were reported in column volumes (CV). Columns were prepared in Biotage ${ }^{\circledR}$ SNAP KP-Sil cartridges using $40-63$ $\mu \mathrm{m}$ silica or $25-40 \mu \mathrm{m}$ silica purchased from Silicycle. ${ }^{1} \mathrm{H}-\mathrm{NMR}$ spectra of small molecules and polymers were recorded on Bruker Avance $600 \mathrm{MHz}$ and $700 \mathrm{MHz}$ spectrometers respectively. Polymer molecular weights and dispersities were analyzed (relative to polystyrene standards) via GPC using a Waters 2695 Separations Module equipped with a Waters 2414 refractive index detector and a Jordi Fluorinated DVB mixed bed column in series with a Jordi Fluorinated DVB $10^{5} \AA$ pore size column. THF with $2 \%$ acetonitrile was used as the eluent at a flow rate of $2.0 \mathrm{~mL} / \mathrm{min}$. Sonication was performed in a Branson Ultrasonic B2800 bath sonicator. Centrifugation of the polymer-SWNT samples was performed using a Beckman Coulter Allegra X-22 centrifuge. UV-Vis-NIR spectra were recorded on a Cary 5000 spectrometer in dual beam mode, using matching 10 mm quartz cuvettes. Thermogravimetric analysis was performed on a Mettler Toledo TGA/DSC 3+, and all measurements were conducted under an argon atmosphere, with sample masses ranging from 0.5 to $1.0 \mathrm{mg}$. Raman spectra were collected using a Renishaw InVia Laser Raman spectrometer, with three different lasers: a $25 \mathrm{~mW}$ argon ion laser (514 
M.Sc. Thesis - C. Shamshoom - McMaster University- Chemistry and Chemical Biology

nm, $1800 \mathrm{~L} / \mathrm{mm}$ grating); a $500 \mathrm{~mW}$ HeNe Renishaw laser (633 nm, $1800 \mathrm{~L} / \mathrm{mm}$ grating); and a $300 \mathrm{~mW}$ Renishaw laser $(785 \mathrm{~nm}, 1200 \mathrm{~L} / \mathrm{mm}$ grating). For the raw SWNT sample dispersed in $\mathrm{CHCl}_{3}$, laser intensity was set to $1 \%$ for $514 \mathrm{~nm}$ and $633 \mathrm{~nm}$, and $10 \%$ for 785 $\mathrm{nm}$. For the polymer-SWNT samples, laser intensity was set to $1 \%$ for all excitation wavelengths. Fluorescence spectra were measured on a Jobin-Yvon SPEX Fluorolog 3.22 equipped with a $450 \mathrm{~W}$ Xe arc lamp, digital photon counting photomultiplier, and an InGaAs detector, also using a $10 \mathrm{~mm}$ quartz cuvette. Slit widths for both excitation and emission were set to $10 \mathrm{~nm}$ band-pass, and correction factor files were applied to account to instrument variations. Photoluminescence maps were obtained at $25{ }^{\circ} \mathrm{C}$, with $5 \mathrm{~nm}$ intervals for both the excitation and emission. 


\section{Appendix B: Synthetic Procedures}

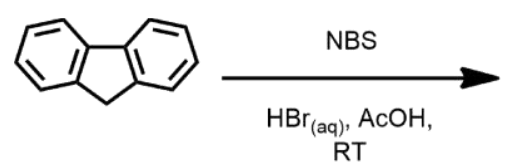<smiles>Brc1ccc2c(c1)Cc1cc(Br)ccc1-2</smiles>

1

$\mathrm{BrC}_{6} \mathrm{H}_{12} \mathrm{Br},{ }^{n} \mathrm{Bu}_{4} \mathrm{NBr}$,

Sat. KOH, Toluene,

$\checkmark$ Reflux

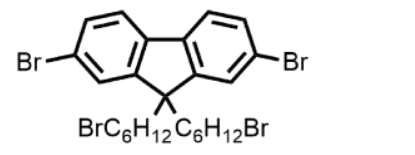

3

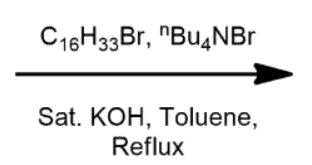

Reflux

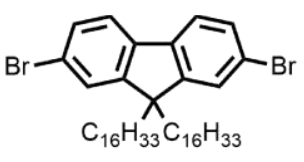

2

$\mathrm{B}_{2} \mathrm{Pin}_{2}, \mathrm{KOAC}$, $\mathrm{Pd}(\mathrm{dppf}) \mathrm{Cl}_{2}$, Dioxane, $80^{\circ} \mathrm{C}$

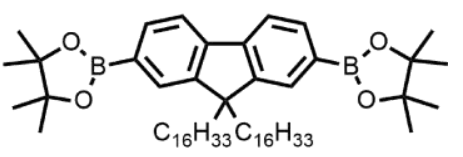

4

Scheme S1. Synthesis of monomers 3 and 4.

2,7-dibromofluorene (1) (prepared according to literature procedures ${ }^{146}$ )

A round bottom flask equipped with a magnetic stir bar was charged with fluorene $(33.2 \mathrm{~g}$, $200 \mathrm{mmol})$, NBS $(89.0 \mathrm{~g}, 500 \mathrm{mmol})$ and acetic acid $(400 \mathrm{~mL})$. While the mixture was stirring, conc. $\mathrm{HBr}(10 \mathrm{~mL})$ was slowly added and then the reaction mixture was stirred at RT for $1.5 \mathrm{~h}$. Water $(200 \mathrm{~mL})$ was added and the resulting suspension was filtered and washed with water to obtain an orange-white solid. The solid was recrystallized from a 1.5:1 v/v mixture of EtOH:acetone ( 1.8 L total volume), and the mother liquor was recrystallized again from the same solvent mixture ( $1.5 \mathrm{~L}$ total volume). The crops were combined to afford $1(41.2 \mathrm{~g}, 64 \%)$ as a colourless solid. ${ }^{1} \mathrm{H}-\mathrm{NMR}\left(600 \mathrm{MHz} ; \mathrm{CDCl}_{3}\right): \delta$ $7.67(\mathrm{~d}, J=1.1 \mathrm{~Hz}, 2 \mathrm{H}), 7.61(\mathrm{~d}, J=8.1 \mathrm{~Hz}, 2 \mathrm{H}), 7.51(\mathrm{dd}, J=8.1,1.8 \mathrm{~Hz}, 2 \mathrm{H}), 3.88(\mathrm{~s}$, $2 \mathrm{H})$. 
M.Sc. Thesis - C. Shamshoom - McMaster University- Chemistry and Chemical Biology

2,7-dibromo-9,9-dihexadecylfluorene (2) (prepared according to literature procedures ${ }^{146}$ ) A round bottom flask equipped with a magnetic stir bar was charged with 1 ( $2.7 \mathrm{~g}, 8.3$ mmol), 1-bromohexadecane (6.36 g, $20.8 \mathrm{mmol}),{ }^{\mathrm{n}} \mathrm{Bu} 4 \mathrm{NBr}(537 \mathrm{mg}, 1.7 \mathrm{mmol})$, toluene $(16.6 \mathrm{~mL})$, and sat. $\mathrm{KOH}(\mathrm{aq})(16.6 \mathrm{~mL})$. The reaction mixture was heated to $60{ }^{\circ} \mathrm{C}$ and stirred vigorously for $1 \mathrm{~h}$ under a nitrogen atmosphere. The biphasic mixture was allowed to separate, and the organic layer was isolated. The aqueous phase was extracted twice with diethyl ether ( $2 \times 20 \mathrm{~mL})$ and the organic extracts were combined and concentrated in vacuo to obtain a viscous green oil. The crude product was purified by flash chromatography (100 g column, 100\% hexanes over $10 \mathrm{CV}$ ) to afford 2 as a colourless solid (5.19 g, 81\%). ${ }^{1} \mathrm{H}-\mathrm{NMR}\left(600 \mathrm{MHz} ; \mathrm{CDCl}_{3}\right): \delta 7.51(\mathrm{~d}, J=8.0 \mathrm{~Hz}, 1 \mathrm{H}), 7.46-7.43(\mathrm{~m}$, 2H), $1.92-1.88(\mathrm{~m}, 2 \mathrm{H}), 1.24-1.03(\mathrm{~m}, 26 \mathrm{H}), 0.88(\mathrm{t}, J=7.0 \mathrm{~Hz}, 3 \mathrm{H}), 0.59-0.57(\mathrm{~m}, 2 \mathrm{H})$.

2,7-dibromo-9,9-bis(6-bromohexyl)fluorene (3) (prepared according to literature procedures $^{146}$ )

A round bottom flask equipped with a magnetic stir bar was charged with 1 (5 g, 15.4 mmol), 1,6-dibromohexane (37.7 g, $154 \mathrm{mmol})$, toluene (31 mL), and sat. $\mathrm{KOH}(31 \mathrm{~mL})$. ${ }^{\mathrm{n}} \mathrm{Bu} 4 \mathrm{NBr}(1.0 \mathrm{~g}, 3.1 \mathrm{mmol})$ was then added and the reaction mixture was heated to $60{ }^{\circ} \mathrm{C}$ and stirred vigorously for $1 \mathrm{~h}$ under a nitrogen atmosphere. The biphasic mixture was allowed to separate, and the organic layer was isolated. The aqueous phase was extracted twice with diethyl ether $(2 \times 120 \mathrm{~mL})$ and the organic extracts were combined and concentrated in vacuo to obtain a viscous green oil. Excess 1,6-dibromohexane was removed using vacuum distillation $\left(1 \mathrm{mbar}, 115^{\circ} \mathrm{C}\right)$ to obtain a viscous yellow oil. The crude mixture was purified by flash chromatography (100 g column, 0 to $20 \% \mathrm{CH}_{2} \mathrm{Cl}_{2}$ in 
M.Sc. Thesis - C. Shamshoom - McMaster University- Chemistry and Chemical Biology

hexanes over $10 \mathrm{CV}$ ) to obtain a colourless solid containing two spots by TLC. The crude product was recrystallized from $\mathrm{MeOH}(\sim 250 \mathrm{~mL})$ to afford 3 as a colourless solid $(4.4 \mathrm{~g}$, 44\%). ${ }^{1} \mathrm{H}-\mathrm{NMR}\left(600 \mathrm{MHz} ; \mathrm{CDCl}_{3}\right): \delta$ 7.53-7.52 (m, 1H), 7.47-7.43 (m, 2H), 3.31-3.28 (t, $2 \mathrm{H}), 1.94-1.91(\mathrm{~m}, 2 \mathrm{H}), 1.68-1.66(\mathrm{~m}, 2 \mathrm{H}), 1.22-1.19(\mathrm{~m}, 2 \mathrm{H}), 1.10-1.07(\mathrm{~m}, 2 \mathrm{H}), 0.60-$ $0.57(\mathrm{~m}, 2 \mathrm{H})$.

\section{2,2'-(9,9-dihexadecylfluorene-2,7-diyl)bis(4,4,5,5-tetramethyl-1,3,2-dioxaborolane)}

(4) (prepared according to literature procedures ${ }^{146}$ )

A round bottom flask equipped with a magnetic stir bar was charged with 2 (5.2 g, 6.7 mmol), $\mathrm{B}_{2} \mathrm{Pin}_{2}(3.76 \mathrm{~g}, 14.8 \mathrm{mmol}), \mathrm{KOAc}(1.98 \mathrm{~g}, 20.2 \mathrm{mmol})$, and dioxane $(28 \mathrm{~mL})$. $\mathrm{Pd}(\mathrm{dppf})_{2} \mathrm{Cl}_{2}(165 \mathrm{mg}, 202 \mu \mathrm{mol})$ was added and then the reaction mixture was stirred at $80{ }^{\circ} \mathrm{C}$ for $12 \mathrm{~h}$. The reaction mixture was partitioned with water and extracted thrice with $\mathrm{Et}_{2} \mathrm{O}$. The organic extracts were combined and dry loaded onto silica (9.9 g). The crude product was purified by flash chromatography (100 g column, 0 to $70 \% \mathrm{CH}_{2} \mathrm{Cl}_{2}$ in hexanes over $10 \mathrm{CV}$ ) to afford 3 as a colourless solid (4.88 g, 63\%). ${ }^{1} \mathrm{H}-\mathrm{NMR}\left(600 \mathrm{MHz} ; \mathrm{CDCl}_{3}\right)$ : $\delta 7.80(\mathrm{~d}, J=7.5 \mathrm{~Hz}, 1 \mathrm{H}), 7.74-7.71(\mathrm{~m}, 2 \mathrm{H}), 2.00-1.97(\mathrm{~m}, 2 \mathrm{H}), 1.39(\mathrm{~s}, 12 \mathrm{H}), 1.24-0.99$ $(\mathrm{m}, 26 \mathrm{H}), 0.87(\mathrm{t}, J=7.0 \mathrm{~Hz}, 3 \mathrm{H}), 0.55-0.53(\mathrm{~m}, 2 \mathrm{H})$.

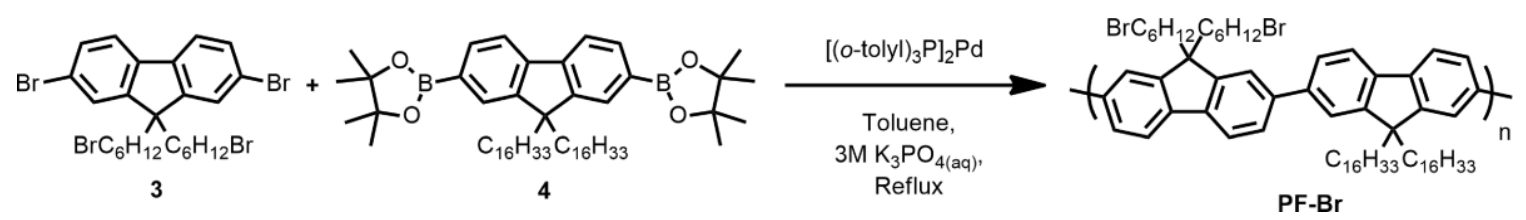

Scheme S2. Synthesis of PF-Br. 
M.Sc. Thesis - C. Shamshoom - McMaster University- Chemistry and Chemical Biology

Poly(dihexadecylfluorene-alt-bis(bromohexyl)fluorene) (PF-Br) (prepared according to literature procedures ${ }^{146}$ )

A Schlenk tube equipped with a magnetic stir bar was charged with $\mathbf{3}$ (1.50 g, $1.73 \mathrm{mmol})$, $4(1.13 \mathrm{~g}, 1.73 \mathrm{mmol})$, toluene $(12.4 \mathrm{~mL})$, and $3 \mathrm{M} \mathrm{K}_{3} \mathrm{PO}_{4}(\mathrm{aq})(12.4 \mathrm{~mL})$. The biphasic mixture was degassed by three freeze pump-thaw cycles, then, while frozen under liquid nitrogen, $\left[(o \text {-tol })_{3} \mathrm{P}\right]_{2} \mathrm{Pd}(62 \mathrm{mg}, 87 \mu \mathrm{mol})$ was added under a positive pressure of nitrogen. The Schlenk tube was evacuated and backfilled with nitrogen four times, and the reaction mixture was vigorously stirred at $80{ }^{\circ} \mathrm{C}$ for $12 \mathrm{~h}$. The phases were allowed to separate, and the organic layer was isolated and filtered through a single plug of celite and neutral alumina. The plug was thoroughly washed with THF and the flow-through was concentrated in vacuo. The crude polymer was precipitated into $\mathrm{MeOH}(400 \mathrm{~mL})$ and then filtered to afford PF-Br as a yellow solid (1.71 g, 89\%). ${ }^{1} \mathrm{H}-\mathrm{NMR}\left(700 \mathrm{MHz} ; \mathrm{CDCl}_{3}\right)$ : $\delta$ 7.85-7.83 (m, 4H), 7.73-7.68 (m, 8H), $3.30(\mathrm{t}, 4 \mathrm{H}), 2.17-2.11(\mathrm{~m}, 4 \mathrm{H}), 1.72-1.69(\mathrm{~m}, 4 \mathrm{H})$, $1.30-1.13(\mathrm{~m}, 60 \mathrm{H}), 0.87(\mathrm{t}, 6 \mathrm{H})$. GPC: $\mathrm{M}_{\mathrm{n}}=31 \mathrm{kDa}, \mathrm{Ð}=2.02$.

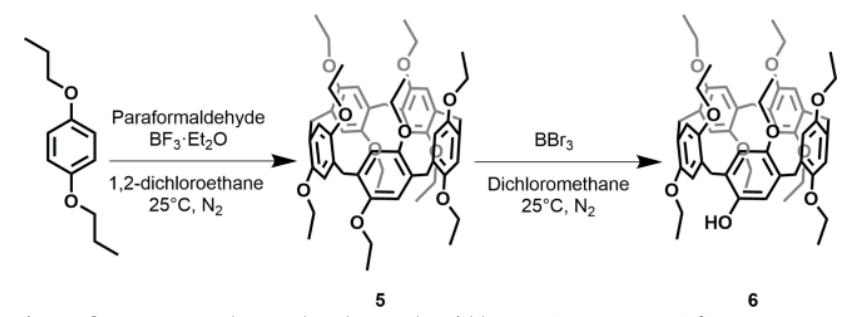

Scheme S3. Synthesis of mono-de-ethylated pillar[5]arene (6).

1,4-bis(ethoxy)pillar[5]arene (5) (adapted from literature procedures ${ }^{166}$ )

A round bottom flask equipped with a magnetic stir bar was charged with 1,4diethoxybenzene (10 g, $60 \mathrm{mmol})$, paraformaldehyde $(5.42 \mathrm{~g}, 180.6 \mathrm{mmol})$, and 1,2dichloroethane $(130 \mathrm{~mL}) . \mathrm{BF}_{3} \cdot \mathrm{Et}_{2} \mathrm{O}(7.43 \mathrm{~mL}, 60.2 \mathrm{mmol})$ was added dropwise to the 
M.Sc. Thesis - C. Shamshoom - McMaster University- Chemistry and Chemical Biology

reaction mixture at RT under a nitrogen atmosphere. The mixture was stirred for $1 \mathrm{~h}$ at $\mathrm{RT}$ before diluting with hexanes $(200 \mathrm{~mL})$ and filtering through a silica plug and washing with 50/50 v/v $\quad \mathrm{CH}_{2} \mathrm{Cl}_{2}$ :hexanes. Fractions containing pillar[5]arene $5\left(\mathrm{R}_{\mathrm{f}} \sim 0.5,70 / 30\right.$ $\mathrm{CH}_{2} \mathrm{Cl}_{2}$ :hexanes) were concentrated in vacuo to afford 5 as a colourless solid (4.3 g, 40\%). ${ }^{1} \mathrm{H}-\mathrm{NMR}\left(600 \mathrm{MHz} ; \mathrm{CDCl}_{3}\right): \delta 6.82(\mathrm{~s}, 10 \mathrm{H}), 3.88(\mathrm{q}, J=7.0 \mathrm{~Hz}, 20 \mathrm{H}), 3.77(\mathrm{~s}, 10 \mathrm{H}), 1.36$ $(\mathrm{t}, J=7.0 \mathrm{~Hz}, 30 \mathrm{H})$.

Mono-de-ethylated Pillar[5]arene (6) (adapted literature procedures ${ }^{166}$ )

A $500 \mathrm{~mL}$ flame-dried round bottom flask equipped with a magnetic stir bar was charged with 5 (1 g, $1.12 \mathrm{mmol})$ and $\mathrm{CH}_{2} \mathrm{Cl}_{2}(120 \mathrm{~mL})$. $\mathrm{A} \mathrm{BBr}$ solution $\left(1 \mathrm{M}\right.$ in $\mathrm{CH}_{2} \mathrm{Cl}_{2} ; 1.0 \mathrm{~mL}, 1$ mmol) was added dropwise to the reaction mixture at RT under a nitrogen atmosphere. The mixture was stirred for 20 min at RT before quenching the reaction with $\mathrm{dH}_{2} \mathrm{O}(\sim 10 \mathrm{~mL})$. The organic phase was extracted with brine, dried with $\mathrm{MgSO}_{4}$, and concentrated in vacuo. The crude product was purified by flash chromatography (100 g column, 5 to $10 \%$ EtOAc in hexanes over $10 \mathrm{CV}$; monitored at $296 \mathrm{~nm}$ ) to afford $\mathbf{6}$ as a colourless solid (194 mg, 20\%). ${ }^{1} \mathrm{H}-\mathrm{NMR}\left(600 \mathrm{MHz} ; \mathrm{CDCl}_{3}\right): \delta 6.90(\mathrm{~s}, 1 \mathrm{H}), 6.78(\mathrm{~s}, 1 \mathrm{H}), 6.70(\mathrm{~s}, 1 \mathrm{H}), 6.69(\mathrm{~s}, 1 \mathrm{H})$ $6.64(\mathrm{~m}, 3 \mathrm{H}), 6.55(\mathrm{~m}, 3 \mathrm{H}), 6.49(\mathrm{~s}, 1 \mathrm{H}), 4.03(\mathrm{q}, J=7.0 \mathrm{~Hz}, 2 \mathrm{H}), 3.92-3.72(\mathrm{~m}, 24 \mathrm{H}), 3.64$ $(\mathrm{q}, \mathrm{J}=7.0 \mathrm{~Hz}, 2 \mathrm{H}), 1.44-1.35(\mathrm{~m}, 10 \mathrm{H}), 1.24-1.07(\mathrm{~m}, 16 \mathrm{H}), 1.03(\mathrm{t}, J=7.0 \mathrm{~Hz}, 3 \mathrm{H})$. 


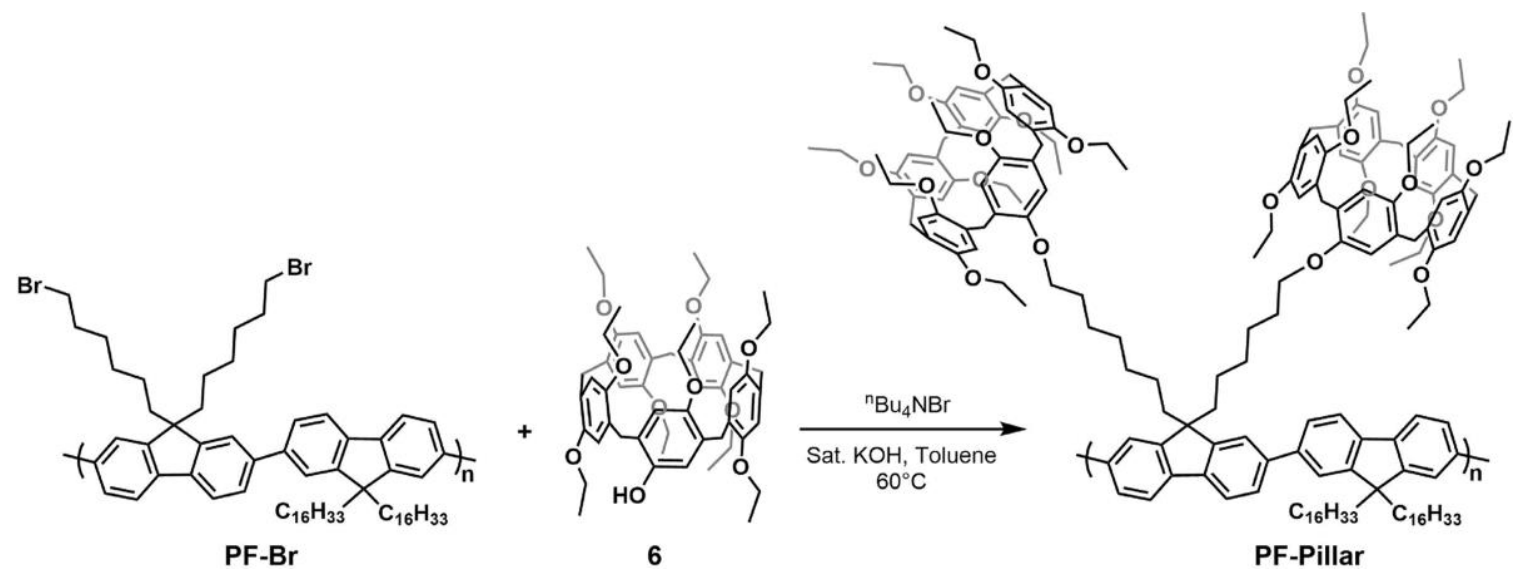

Scheme S4. Synthesis of PF-Pillar.

\section{Poly(dihexadecylfluorene-alt-bis(pillar[5]arene)fluorene) (PF-Pillar)}

A round bottom flask equipped with a magnetic stir bar was charged with PF-Br (50 mg, $45 \mu \mathrm{mol}), 6$ (97.8 mg, $110 \mu \mathrm{mol}),{ }^{\mathrm{n}} \mathrm{Bu} 4 \mathrm{NBr}(5.8 \mathrm{mg}, 20 \mu \mathrm{mol})$, toluene (400 uL) and sat. $\mathrm{KOH}(400 \mathrm{uL})$. The reaction mixture was stirred for $12 \mathrm{~h}$ at $60^{\circ} \mathrm{C}$. The reaction mixture was cooled to RT, filtered through an alumina plug and precipitated into $\mathrm{MeOH}(\sim 20 \mathrm{~mL})$. The suspension was filtered and dried by vacuum to afford PF-Pillar as a yellow solid (61 mg, 50\%). ${ }^{1} \mathrm{H}-\mathrm{NMR}\left(700 \mathrm{MHz} ; \mathrm{CDCl}_{3}\right): \delta$ 7.87-7.69 (m, 12H), 6.78-6.66 (m, 20H), 3.84$3.63(\mathrm{~m}, 60 \mathrm{H}), 2.21-2.13(\mathrm{~m}, 7 \mathrm{H}), 1.56-1.13(\mathrm{~m}, 90 \mathrm{H})$. 
M.Sc. Thesis - C. Shamshoom - McMaster University- Chemistry and Chemical Biology

\section{Appendix C: Calculations and Figures}

\section{Calculation of SWNT Concentration by TGA}

The relevant data to calculate SWNT concentration are tabulated in Table S1. The mass losses, which correspond to polymer side chain degradation, were used to calculate the SWNT mass fraction $\left(f_{S W N T}\right)$. Given that the SWNT mass loss is negligible under the experimental conditions, the mass fraction of the polymer $\left(f_{\text {polymer }}\right)$ can be calculated according to Eq. S1:

$$
\mathrm{f}_{\text {polymer }}=\frac{\varphi_{\text {polymer }-S W N T}}{\varphi_{\text {polymer }}}
$$

Where $\varphi_{\text {polymer }}$ is the mass loss of polymer and $\varphi_{\text {polymer-SWNT }}$ is the mass loss of the polymer-SWNT complex. $f_{S W N T}$ can then be calculated accordingly, given that $f_{\text {polymer }}+$ $f_{S W N T}=1$. For the PF-Pillar-SWNT dispersion, $V_{\text {polymer-SWNT }}$ was $0.5 \mathrm{~mL}, m_{\text {polymer-SWNT }}$ was $0.5 \mathrm{mg}$, and $f_{\text {SWNT }}$ was 0.60 . The SWNT concentration $\left(c_{S W N T}\right)$ was calculated according to Eq. S2:

$$
\begin{array}{r}
c_{S W N T}=\frac{f_{S W N T} \times m_{\text {polymer }-S W N T}}{V_{\text {polymer }-S W N T}} \\
c_{S W N T}=\frac{0.6 \times 0.5 \mathrm{mg}}{0.5 \mathrm{~mL}} \\
c_{S W N T}=600 \mu \mathrm{g} \cdot \mathrm{mL}^{-1}
\end{array}
$$

Table S1. Tabulated Data for SWNT Concentration Calculations.

\begin{tabular}{ccc}
\hline & PF-Br & PF-Pillar \\
\hline$\varphi_{\text {polymer }}$ & $65.2 \%$ & $50.1 \%$ \\
$\varphi_{\text {polymer-SWNT }}$ & $38.1 \%$ & $20.1 \%$ \\
$f_{S W N T}$ & 0.42 & 0.60 \\
$V_{\text {polymer-SWNT }}$ & $6 \mathrm{~mL}$ & $0.5 \mathrm{~mL}$ \\
$m_{\text {polymer-SWNT }}$ & $0.3 \mathrm{mg}$ & $0.5 \mathrm{mg}$ \\
\hline
\end{tabular}


M.Sc. Thesis - C. Shamshoom - McMaster University- Chemistry and Chemical Biology

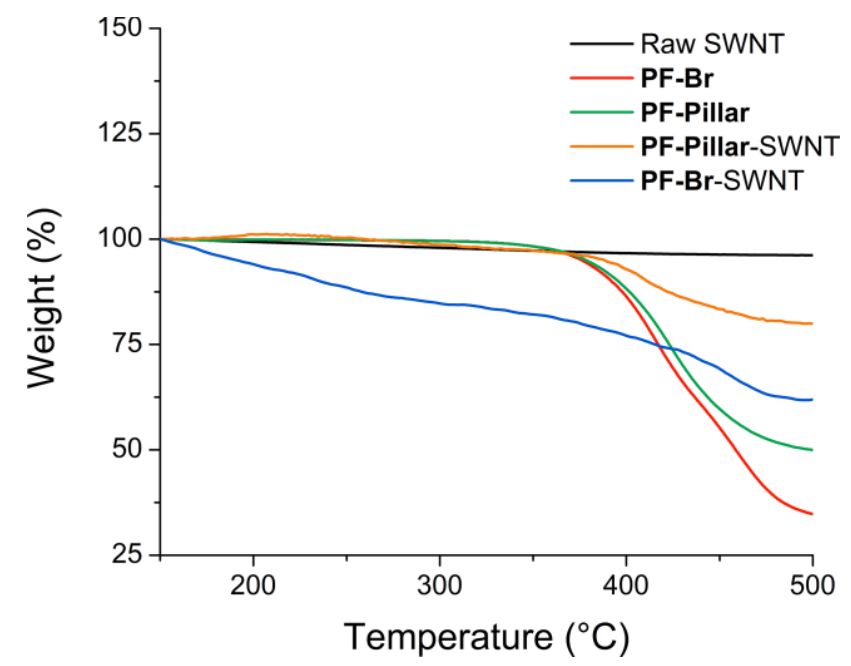

Figure S1. TGA thermograms of PF-Pillar (green), PF-Br (red), PF-Pillar-SWNT (orange), PF-Br-SWNT (blue), and Raw HiPCO SWNTs (black).
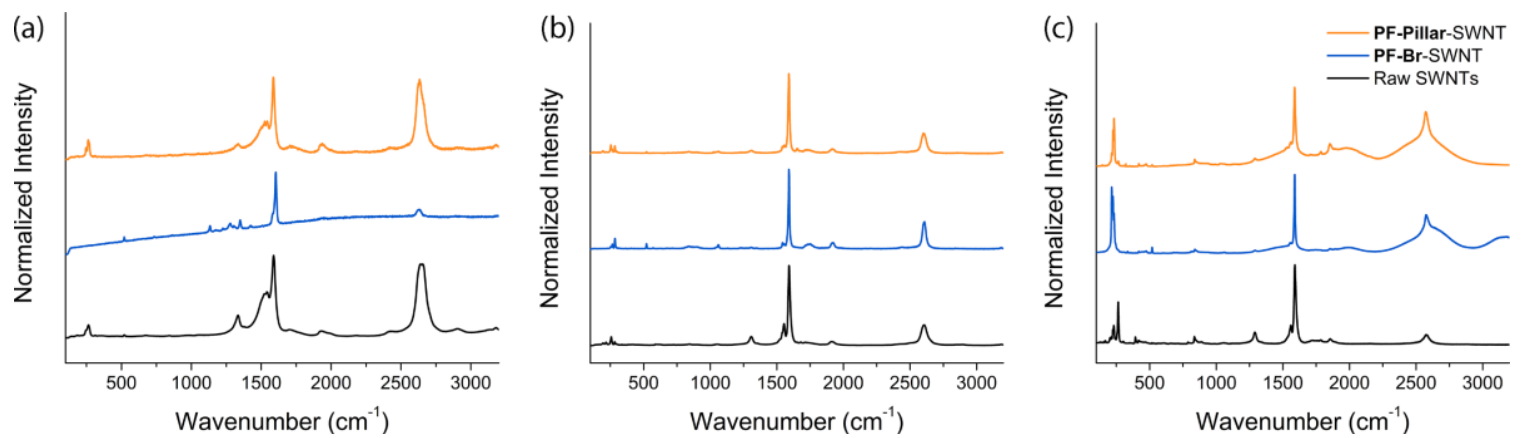

Figure S2. Full Raman spectra of raw HiPCO and polymer-SWNT samples at (a) 514, (b) 633, and (c) $785 \mathrm{~nm}$ excitation wavelengths. The spectra were normalized to the G-band at $\sim 1590 \mathrm{~cm}-1$ and offset for clarity. 
M.Sc. Thesis - C. Shamshoom - McMaster University- Chemistry and Chemical Biology

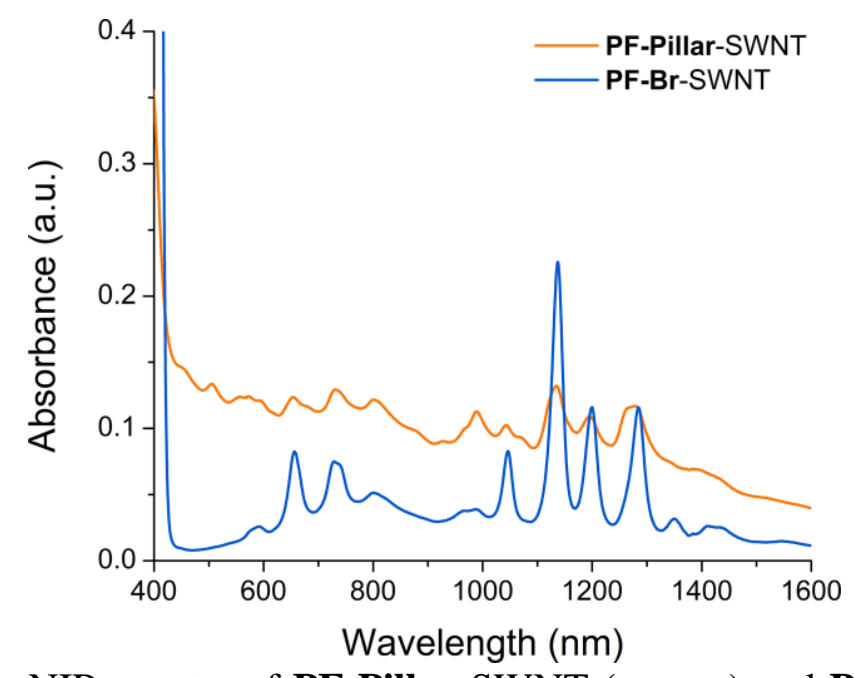

Figure S3. UV-Vis-NIR spectra of PF-Pillar-SWNT (orange) and PF-Br-SWNT (blue) used for PL mapping.
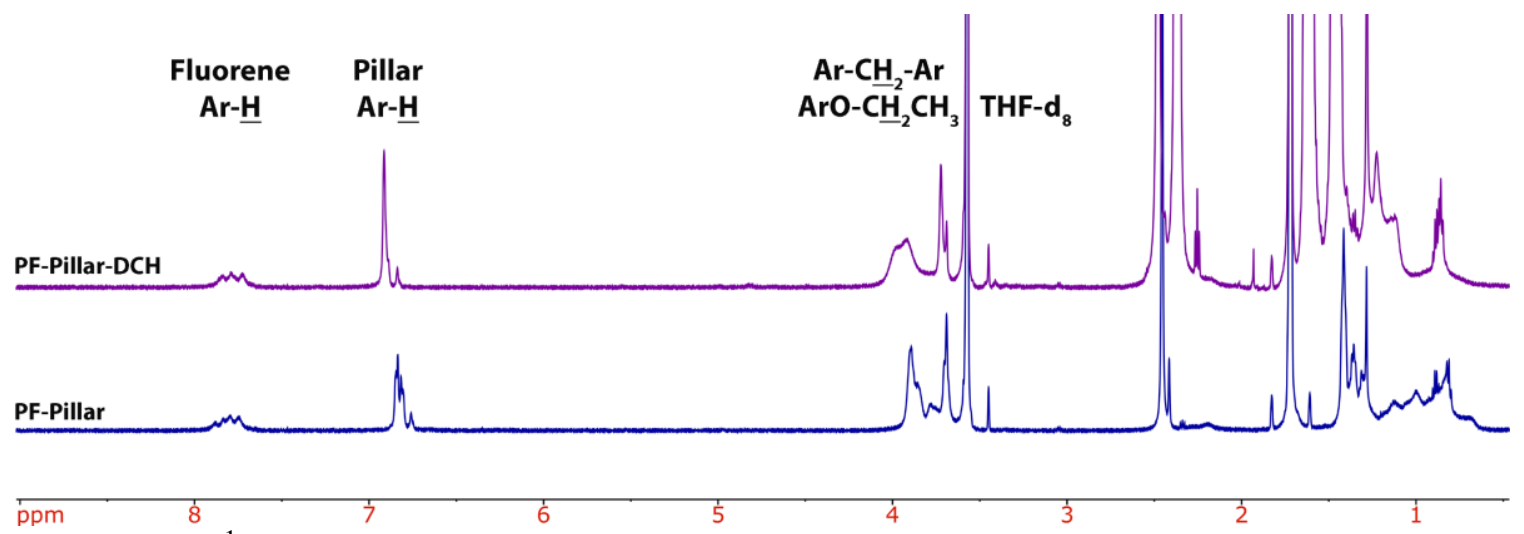

Figure S4. ${ }^{1} \mathrm{H}-\mathrm{NMR}$ spectra (THF-d8, $298 \mathrm{~K}$ ) of PF-Pillar (blue) recorded after the addition of 78 eq of 1,6-dicyanohexane ( $\mathrm{DCH}$; purple). 
M.Sc. Thesis - C. Shamshoom - McMaster University- Chemistry and Chemical Biology

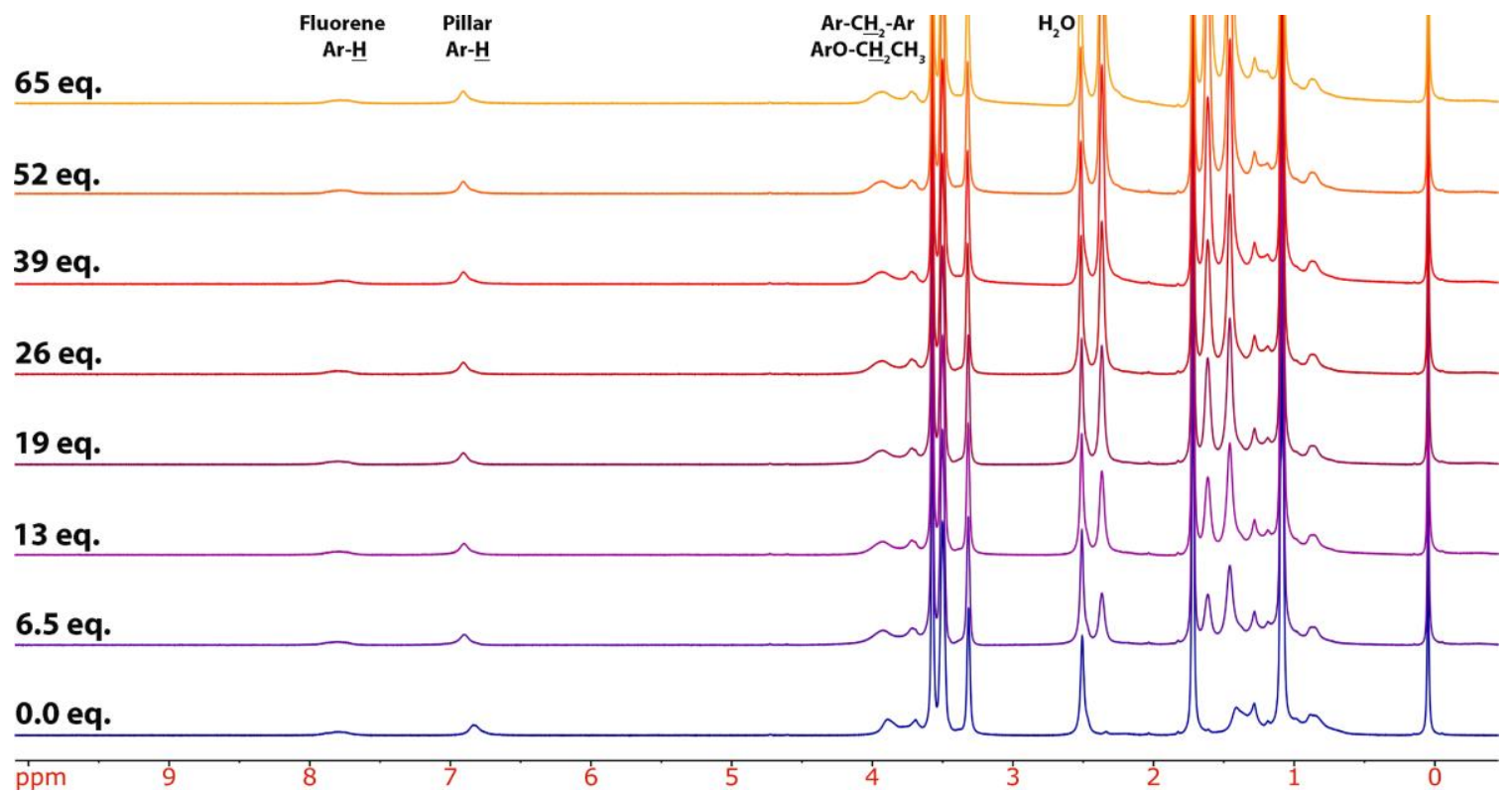

Figure S5. Full ${ }^{1} \mathrm{H}-\mathrm{NMR}$ spectra (THF-d8, $298 \mathrm{~K}$ ) of the PF-Pillar-SWNT dispersion recorded after successive additions of 1,6-dicyanohexane $(0-65 \mathrm{eq})$.

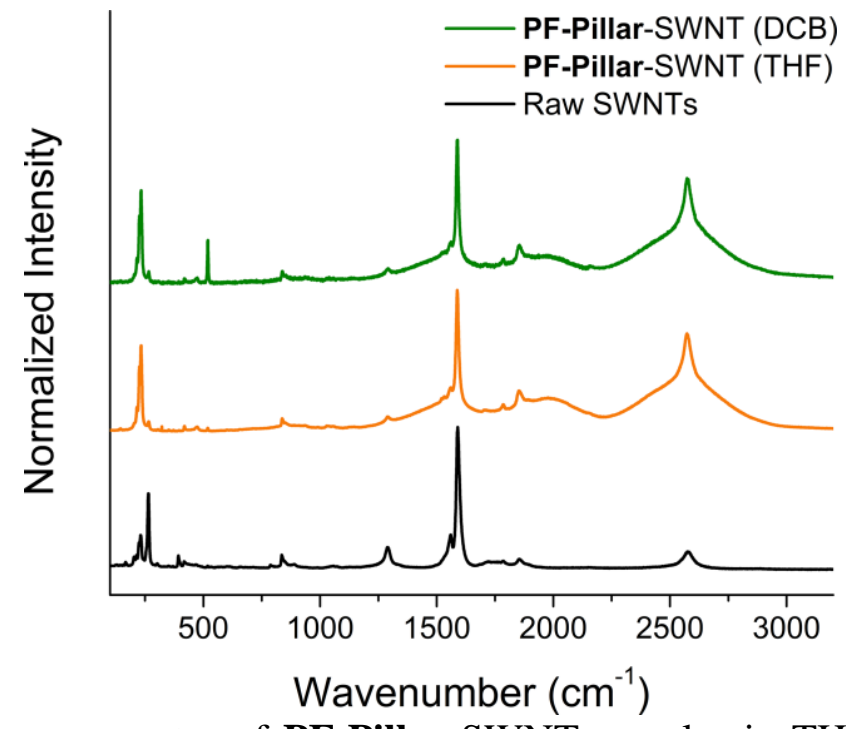

Figure S6. Full Raman spectra of PF-Pillar-SWNT samples in THF and upon solvent exchange into 1,2-dichlorobenzene (DCB), compared with raw HiPCO SWNTs. The spectra were collected at $785 \mathrm{~nm}$ excitation wavelength, normalized to the G-band at $~ 1590$ $\mathrm{cm}-1$ and offset for clarity. 